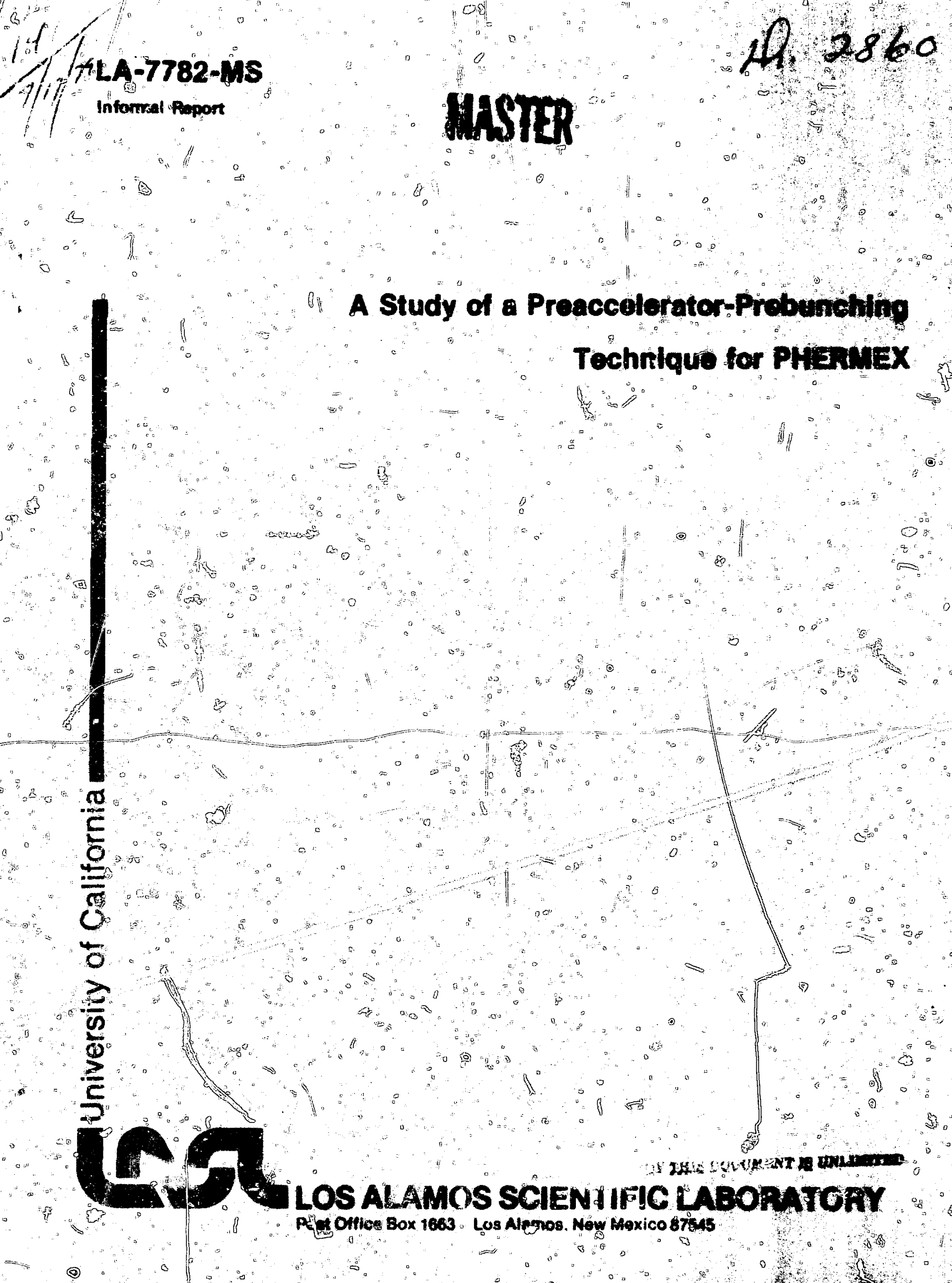


LA-7782-MS

Informal Report

UC-28

Issued: May 1979

\title{
A Study of a Preaccelerator-Prebunching Technique for PHERMEX
}

\author{
Reynałdo Morales \\ Douglas Venable
}

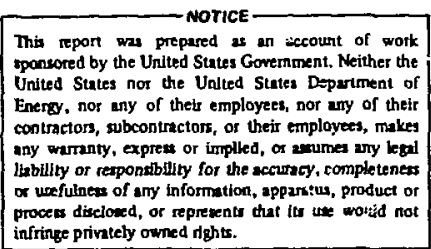

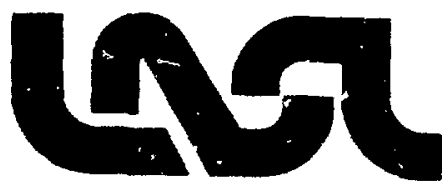




\title{
A STUdY OF A PREACCELERATOR-PREBUNCHING TECHNIQUE FOR PHERMEX
}

\author{
by
}

\author{
Reynaldo Morales and Douglas Venabie
}

\begin{abstract}
A feasible, 1-m-long, 50-MHz, preaccelerator bunching cavity excited to an attainable field of $10 \mathrm{MV} / \mathrm{m}$ is shown to be suitable for injecting sequences of high-current, 5-ns, electron bunches into PHERMEX in a way that increases the PHERMEX output flux by factors of 2 to 5 . This result can be attributed to an increase in energy of the ejected electrons, an increase in the amount of charge that PHERMEX can accept, more efficient use of the electromagnetic energy stored in the PHERMEX accelerating structures, and a substantial increase in the electron optical efficiency in delivering the accepted injected electron beam to the target with the most favorable momentum distribution there.
\end{abstract}

\section{INTRODUCTION}

With a sufficiently enhanced radiation output from PHERMEX' (Pulsed High-Energy Radiographic Machine Emitting $X$ Rays) or the radiographic machine associated with PUFF (Pulsed Ultrahigh Flux Facility), multiple-pulse flash radiography becomes very attractive. This report examines some of the critical features of one method for achieving multiple-pulse injection with conventional state-of-the-art techniques. Not only does multiple-frame radiography prove to be practical, but this particular approach also has several attractive spin-offs.

One spin-off is the capability to inject a smalldiameter, high-current, electron beam whose small diameter can be maintained throughout. More of such a beam ejected from PHERMEX can be focused to a smaller spot on the target, with a convergence angle less than the natural bremsstrahlung $\mu / V$ where $\mu$ is the electron rest mass energy and $V$ is the beam energy.

For a fixed injected charge, the on-axis radiation intensity varies with the 2.8 power of $V$, which is the sum of the injection eriergy $V_{1}$ and the energy gain of the accelerator $V_{\mathrm{g}}$. Therefore, another spin-off is the substantial increase in radiation through the contribution of $V_{i}$ to $V$. We examine this effect in more detail in Appendix A. Variation of on-axis radiation intensity with the 2.8 power of $V$ has been experimentally verified by T.H. Martin ${ }^{2}$ on the basis of PHERMEX data at $27 \mathrm{MeV}$. He also demonstrated the importance of having a beam of nearly zero convergence impinging upon the target. Small average angles of arrival at the target of electrons whose voltages exceed $13 \mathrm{MeV}$ could seriously affect the dose output measured at $1 \mathrm{~m}$. For example, at $20 \mathrm{MeV}$, a 2-degree average electron-beam divergence can cause a 20 to $30 \%$ decrease in the dose measured at $1 \mathrm{~m}$.

Another spin-off is the fact that, the higher the energy of the particles injected into PHERMEX, the greater the accoptance phase angle, meaning that more charge can be injected that can be delivered to the target. For instance, at an injection energy of $V_{i}=0.5 \mathrm{MeV}$ and an accelerating field strength of $\sim 5 \mathrm{MV} / \mathrm{m}$, the acceptance angle is about $105^{\circ}$, whereas, for $V_{1}=10 \mathrm{MeV}$, it is effectively about 270 . A sharply bunched beam, which is obtainable with a light-modulated cathode or by the technique 
described here, also permits one to inject more charge that can reach the target than is possible by injecting continuously over each if cycle, as currently practiced.

We considered an existing electron-gun design with perveance $\left(P=I V^{-3 / 2}\right)$ of roughly $10^{-6}$ that will yield about $1000 \mathrm{~A}$ at about $1 \mathrm{MeV}$. The gun can be pulsed at this voltage with state-of-the-art pulsers to produce a programmed series of 40 -ns-long pulses, corresponding to two if cycles. These bunches of electrons are then injected into a preaccelerator if $(50-\mathrm{MHz})$ cavity to chop it into 1000-A, 5-ns-long bursts. The convergence of the beam ejected froin this cavity is corrected by stateof-the-art magnetic lenses so that these short bursts of electrons are injected into PHERMEX at a small diameter and are approximately parallel to the axis.

The block diagram, in Fig. 1 illustrates the arrangement. The basic considerations of particular concern are the effects of changes in convergence because of space charge and changes at various cavity apertures in this injection system. Not shown are other features of the injector that are available commercially or are well within current practice. For example, the $100 . \mathrm{mm}$ gun now in use would very nearly satisfy the requirements of the clectron source; the multiple-pulse pulser, although not extant, can be manufactured commercially and it need not be developed here. A high-field, 50-MHz cavity can be designed and fabricated at a number of places. Later, we suggest that a nominal field strength of $10 \mathrm{MV} / \mathrm{m}$ is attainable. The rf-power amplifiers for driving this cavity can be identical with those we are using; recent Los Alamos Scien- tific Laboratory (LASL) developments, however, have demonstrated that our new rf amplifier will be even better; at least three times more rf power, with much higher powers ultimately expected, and at less cost. That we can achieve this field strength without end-to-end electrical breakdown has also been demonstrated; we achieved more than 16 $\mathrm{MV} / \mathrm{m}$ in a shorter cavity during some of the early work on PHERMEX-like systems.

\section{Calculational objectives}

Our principal study objectives were to demoristrate that

(1) When using conventional electron optical techniques, it is practical to inject into PHERMEX an electron beam whose current and energy are high enough for total PHERMEX output radiation to be increased to at least several hundred roentgens.

(2) This radiation can be divided into at least three $40-n s$ pulses of about $100 \mathrm{R}$ each by using a commercially available pulser driving an existing electron injector (the $100-\mathrm{mm}$ PHERMEX gun).

To demonstrate these objectives, we need only (1) a physically consistent set of engineering parameters that govern the electron gun with its accompanying magnetic lenses and its driving pulser, (2) the preaccelerator, (3) the optical properties of the bunched electron beam that is ejected from the preaccelerator, and (4) the lens scheme that shapes this ejected beam and injects it into the first cavity of PHERMEX. Of the various

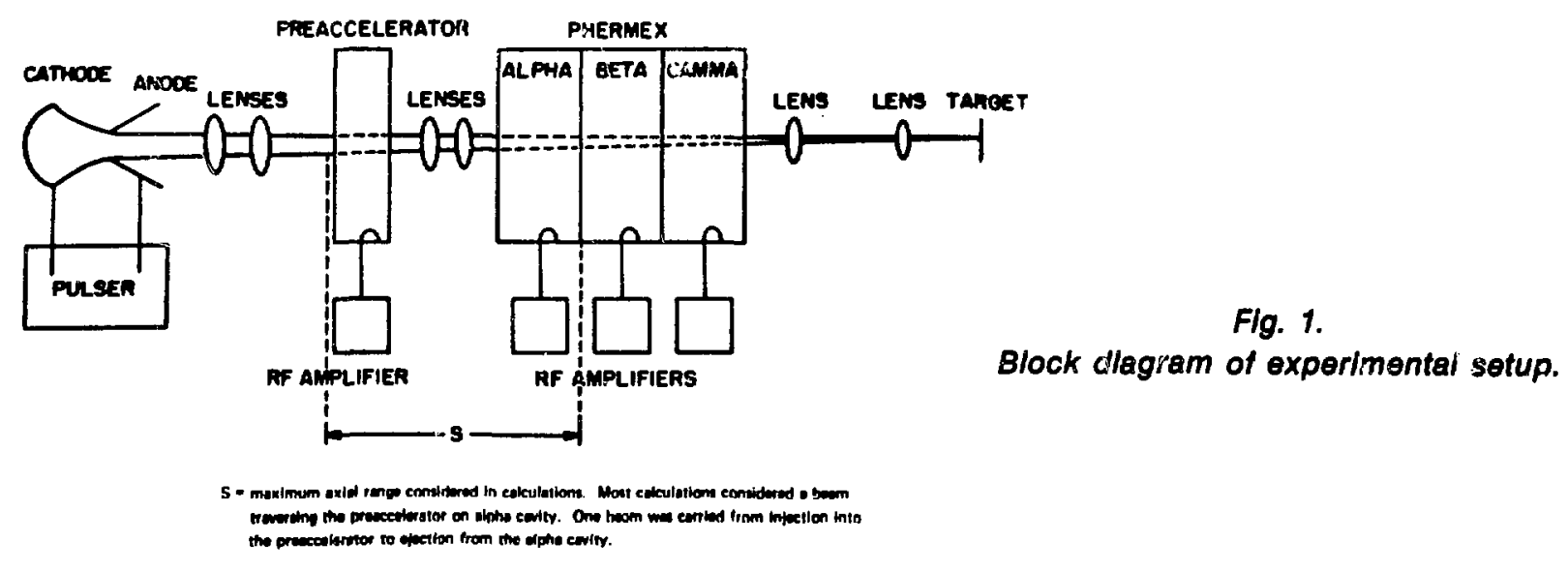


components, the gun puiser, the electron gun, its magnetic lenses, the beam-shaping lenses, and the preaccelerator's rf accelerating cavity are viewed as being well within the state of the art. With our present technology, we can assume that these components may be had just for the effort and funding. Roughly summarized, this study involved consideration of the variables that, for a given conceptual design, govern the accessibility to PHERMEX target of the electrons injected into PHERMEX.

The problem boils down to studies of beam trajectories (and therefore, beam convergences), energy and momentum spectra, and beam-curren: density distributions as functions of several crucial positions along the optical axis. Tne practicality argument here consists only of satisfying the concern that, in addition to being attainable, none of these parameters is critically related to its independent variables.

We satisfied this concern by extensive numerical exploration of engineering-feasible cases that consisted initially of elaborating on previous caiculations reported in Ref. 1. We made no attempt to optimize any parameters for subsequent design purposes, and we present no cost-effectiveness arguments in this report.

R. Morales made substantial modifications of the GRAPE SEED code to accommodate the variety of problems.

Tabje I lists the basic input data for the several problems we treated. Included are beams with initial convergences well within the range of present electron optics. Detailed results of all these calculations are given in Appendix B; in the main body of the report, we merely present results of typical problems that support our calculational nbjectives.

\section{RESULTS}

Typical of our calculations are the data presented in Figs. 2 through 5 . A 2-cm-radius, 2000-A, 1-MeV beam wes injected with zero convergence intc the $1-\mathrm{m}$-long preaccelerator cavity excited to $10 \mathrm{MV} / \mathrm{m}$ in Case 1 shown in these figures and, in Case 3, into the 2.6-m-long first cavity of PHERMEX also excited to $10 \mathrm{MV} / \mathrm{m}$. In Case 2 the output beam from the 1-m-long cavity was then shaped by conventional optics and in- jected into the 2.6-m-long cavity located $1 \mathrm{~m}$ away. Computationally, we did this by assuming that a lens existed throughout the 1-in drift space that kept $\dot{A}$ equal to zero (prevented beam expansion). Improved results could be obtained by using lenses that focus the beam down.

Figure 2 illusirates the beam-current (or beamcharge) density Jistribution upon ejection of the beam from the 1-m cavity (Case 1 ) and the 2.6-m cavity (Cases 2 and 3 ). Figure 3 demonstrates that $90 \%$ of the beam in Case 2 lies within a $2.5-\mathrm{cm}$ radius; in Case 1 , within a $7.3-\mathrm{cm}$ radius; and in Case 3, within an $8.7-\mathrm{cm}$ radius. A far more tightly confined beam emerges from the $2.6-\mathrm{cm}$ cavity when coupled with the preaccelerator than without it. Figure 4 illustrates the energy spectra of the emerging beam for those three cases. Note that the beam emerging from the $1-m$ cavity peaks rather flatly at about $10 \mathrm{MeV}$ and that the energy of the beam from the $26-\mathrm{cm}$ caviiy peaks at about 20 $\mathrm{MeV}$ with only a $1-\mathrm{MeV}$ beam injected, but at 27 $\mathrm{MeV}$ when the output of the $1-\mathrm{m}$ cavity is injected into the $2.6-\mathrm{cm}$ cavity. The peak energy can be further improved by adjusting the range of the injection phase angle of the $5-\mathrm{ns}, 10-\mathrm{MeV}$ bunches injected into the $2.6-\mathrm{cm}$ cavity. The energy depletion over one vycle is about $8 \%$ in Case $1,5 \%$ in Case 2, and $7 \%$ in Case 3.

One pleasant surprise in these particular calculations was the demonstration that, over the spectral region where the beam energy is the highest, the beam convergence upon ejection varies only weakly with time, as shown in Fig. 5 .

The 7-MeV gain $27 \mathrm{MeV}$ minus $20 \mathrm{MeV}$, corresponding to preaccelerator injection less conventional) contributes substantially to the PHERMEX output flux. Here, corresponding to four, three, and two amplifiers, we assume 10 MVim in a cavity, 8.5 in $\beta$, and 7 in $y$, respectively. Without the preaccelerator, ti,e peak output beam energy would have been about $50 \mathrm{MeV}$, including a 1-MeV injection energy. On the other hand, with the preaccelerator, this peak energy would have been $57 \mathrm{MeV}$. The energy retio raised to the 2.8 power is 1.4 , indicaling a $40 \%$ increase in radiation flux attributed to energy alone. Since more charge is acceptable and can be useful if injected at higher energies, we can have still another increase in this output fiux, in this case by a factor of about 1.3. Thus, these two mechanisms together erhance flux 
TABLE I

BEAM STUDY PARAMETERS

Injected Beam

\begin{tabular}{|c|c|c|}
\hline $\begin{array}{c}\text { Injection } \\
\text { Energy } \\
(\mathrm{MeV}) \\
\end{array}$ & $\begin{array}{l}\text { Iujected } \\
\text { Current } \\
\therefore \text { (imp) }\end{array}$ & $\begin{array}{l}\text { Initial } \\
\text { Radius } \\
\mathbf{R}_{\mathrm{o}} \text { (cm) } \\
\end{array}$ \\
\hline 0.6 & 300 & 2.0 \\
\hline 0.6 & 300 & 2.0 \\
\hline 0.6 & 300 & 2.0 \\
\hline 0.6 & 300 & 2.0 \\
\hline 0.6 & 300 & 2.0 \\
\hline 0.6 & 300 & 2.0 \\
\hline 0.6 & 300 & 2.0 \\
\hline 0.6 & 300 & 2.0 \\
\hline$: . n$ & 600 & 2.0 \\
\hline 1.0 & 600 & 2.0 \\
\hline 1.0 & 100 & 2.0 \\
\hline 1.0 & 1000 & 2.0 \\
\hline 0.6 & 2000 & 2.0 \\
\hline 0.6 & 2000 & 2.0 \\
\hline 0.6 & 2000 & 2.0 \\
\hline 1.0 & 2000 & 2.0 \\
\hline 1.0 & 2000 & 2.0 \\
\hline 1.0 & 2000 & 2.0 \\
\hline 1.0 & 2000 & 2.0 \\
\hline 1.0 & 2000 & 2.0 \\
\hline 1.0 & 2000 & 2.0 \\
\hline 1.0 & 2000 & 2.0 \\
\hline 1.0 & $200 \%$ & 2.0 \\
\hline 8.0 & 1000 & 2.0 \\
\hline 8.0 & 2000 & 2.0 \\
\hline 1.0 & 2000 & 2.0 \\
\hline
\end{tabular}

Cavity Parameters

\begin{tabular}{|c|c|c|c|c|c|c|c|}
\hline $\begin{array}{c}\text { Cavity } \\
\text { Length } \\
\text { (m) } \\
\end{array}$ & $\begin{array}{c}\text { Field } \\
\text { Strength } \\
\text { E (MV/m) }\end{array}$ & $\begin{array}{c}\text { Total Chargis } \\
\text { at End } \\
\text { of Cavity } \\
4(\mu \mathrm{C})\end{array}$ & $\begin{array}{c}\text { Fraction of } \\
\text { Charge } \\
\text { Within } \\
5-\mathrm{cm} \\
\text { Radiug } \\
\end{array}$ & $\begin{array}{c}\text { Fraction of } \\
\text { C!...tse } \\
\text { Within } \\
\text { 2.5-em } \\
\text { Radius } \\
\end{array}$ & $\begin{array}{c}\text { Peak } \\
\text { Energy } \\
(\mathrm{MeV}) \\
\end{array}$ & $\begin{array}{l}\text { Acceptance } \\
\text { Window } \\
\text { (deg) } \\
\end{array}$ & $\begin{array}{c}\text { Enersy } \\
\text { Depletion } \\
\text { Over One } \\
\text { Cycle } \\
\Delta U / U(\%)\end{array}$ \\
\hline 1.0 & 6 & 3.1 & 0.90 & 0.77 & 6.3 & $\sim 185$ & 2.2 \\
\hline 1.0 & 6 & 3.1 & 0.91 & 0.81 & 6.3 & $\sim 185$ & 2.2 \\
\hline 1.0 & 6 & 3.1 & 0.92 & 0.83 & 6.3 & $\sim 185$ & 2.2 \\
\hline 1.0 & 6 & 3.1 & 0.92 & 0.84 & 6.3 & $\sim 185$ & 2.2 \\
\hline 1.0 & 6 & 3.1 & 0.90 & 0.77 & 6.3 & $\sim 185$ & 2.2 \\
\hline 1.0 & 6 & 3.1 & 0.88 & 0.70 & 6.3 & $\sim 185$ & 2.2 \\
\hline 1.0 & 6 & 3.1 & 0.87 & 0.63 & 6.3 & $\sim 185$ & 2.2 \\
\hline 1.0 & 5.4 & 3.1 & 0.90 & 0.75 & 5.7 & $\sim 185$ & 2.5 \\
\hline 1.0 & 6 & 6.2 & 0.90 & 0.74 & 6.7 & $\sim 185$ & 4.6 \\
\hline 1,0 & 10 & 6.2 & 0.94 & 0.86 & 10.5 & $\sim 185$ & 2.6 \\
\hline 1.0 & 6 & 10.3 & 0.83 & 0.60 & 6.7 & $\sim 185$ & 7.5 \\
\hline 1.0 & 10 & 10.3 & 0.91 & 0.79 & 10.5 & $\sim 185$ & 4.2 \\
\hline 1.0 & 6 & 20.6 & 0.50 & 0.13 & 6.4 & $\sim 185$ & 14.0 \\
\hline 1.0 & 6 & 20.6 & 0.45 & 0.11 & 6.4 & $\sim 185$ & 14.0 \\
\hline 1.0 & 10 & 20.6 & 0.75 & 0.46 & 10.1 & $\sim 185$ & 7.7 \\
\hline 1.0 & 4 & 20.6 & 0.61 & 0.16 & 4.9 & $\sim 185$ & 22.0 \\
\hline 1.0 & 4 & 20.6 & 0.67 & $0: 19$ & 4.9 & $\sim 185$ & 22.0 \\
\hline 1.0 & 4 & 20.6 & 0.51 & 0.12 & 4.9 & $\sim 185$ & 22.0 \\
\hline 1.0 & 7 & 20.6 & 0.75 & 0.42 & 7.7 & $\sim 185$ & 12.5 \\
\hline 1.0 & 10 & 20.6 & 0.83 & 0.64 & 10.5 & $\sim 185$ & 8.4 \\
\hline 1.0 & 10 & 20.6 & 0.85 & 0.69 & 10.5 & $\sim 180^{\circ}$ & 8.4 \\
\hline 1.0 & 10 & 20.6 & 0.82 & 0.58 & 10.5 & $\sim 185$ & 8.4 \\
\hline 2.6 & 10 & 15.0 & 0.76 & 0.37 & 19.7 & $\sim 135$ & 7.0 \\
\hline 2.6 & 10 & 8.6 & 1.00 & 0.97 & 26.6 & $\sim 155$ & 4.5 \\
\hline 2.6 & 10 & 17.2 & 1.00 & 0.95 & 26.6 & $\sim 155$ & 9.0 \\
\hline 2.6 & 10 & 16.7 & 0.99 & 0.88 & 27.0 & $\sim 150$ & 5.3 \\
\hline
\end{tabular}




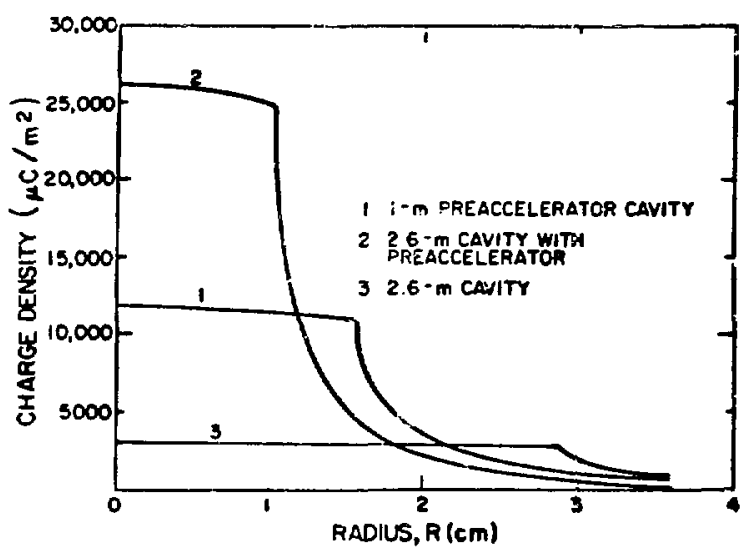

Fig. 2.

Charge density vs radius from beam center.

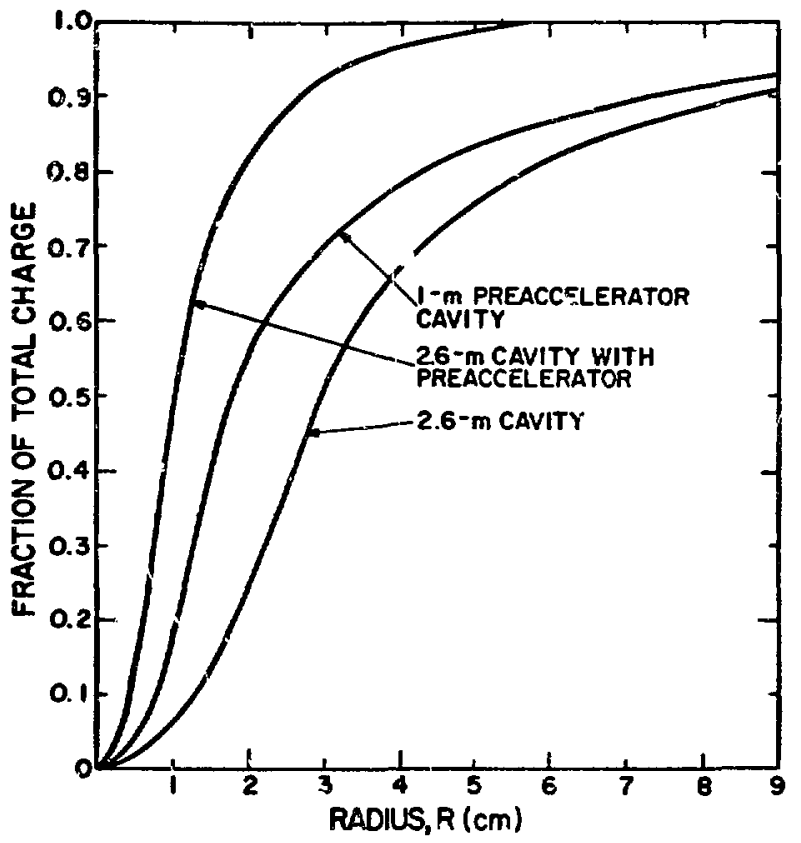

Fig. 3.

Fraction of iotal chargo hitting downstream wall within given radius.

by a factor of about 1.8. Yel another gain is attribuied to maintaining $\varepsilon$ tighter beam as it goes through the three PHEAMEX cayities, hence, delivering more charge to the target. We have not calculated this increase in charge-delivery officiency in detail, as the calculation would require running GRAPE SEED through all cavities, the drift tube, and to the target, a rather itme-consuming

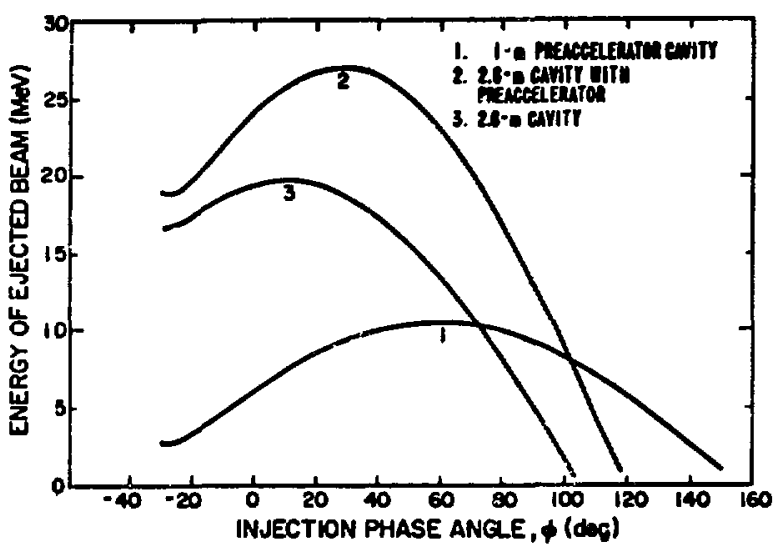

Fig. 4.

Energy of ejected beam vs injection phase angie.

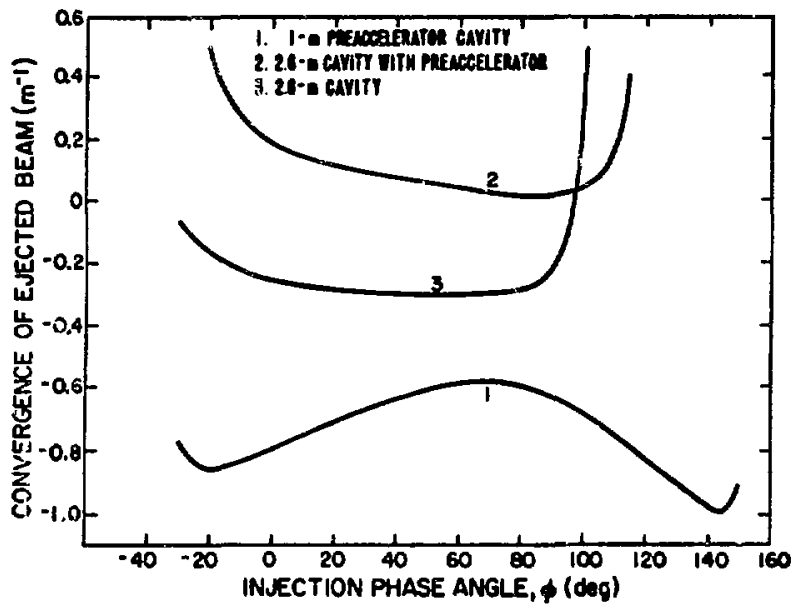

Fig. 5.

Convergence of ejected beam vs iniection phase angle.

operation. We do estimate on the basis of simple lens laws, however, that the gain here would amount to another facter of about 1.5 .

Consideration of the radial component of Lorentz' force equation, expressed in cylindrical coordinates, s"jggests a useful scaling concept. Accounting for space charge and relativistic effects, the radial force on electrons in an unaccelerated cylindrical stream of electrons of.energy $V$ varies as

$\frac{1}{R}\left(\frac{1-\beta^{2}}{\beta}\right)$. 
where $I$ is the beam current enclosed within a beam of radius $R$, and $\left(1-\beta^{2}\right) / \beta$ is the correstion term, which can be expressed in terms of the beam energy and the electron's rest energy, $\mu=0.511$ MeV, as

$$
\left\{\left(\frac{V}{\mu}+1\right)\left[\left(\frac{V}{\mu}+1\right)^{2}-1\right]^{1 / 2}\right\}^{-1}
$$

and as shown in Fig. 6.

For example, we compare a 2000-A, 10-MeV beam injected Irito PHERMEX with the nominal

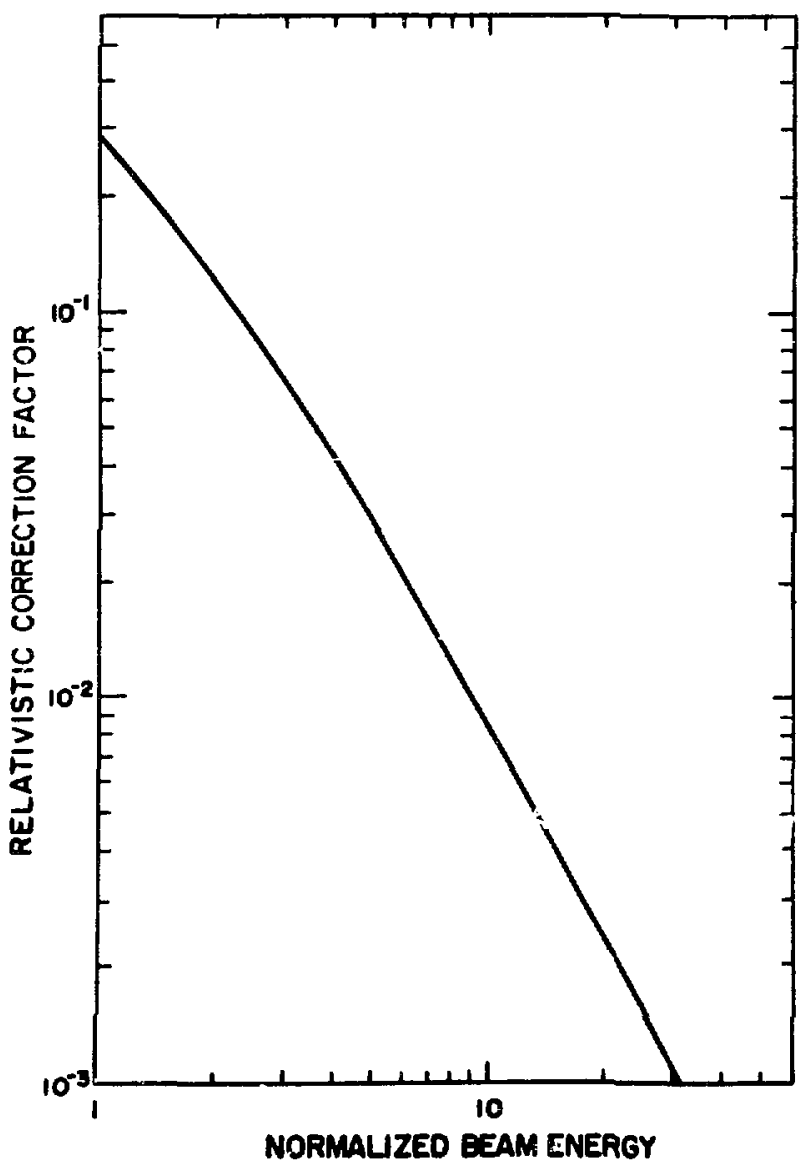

Fig. 6.

Relativistic correction factor ve normallzed beam energy.
300-A, C.5-MeV, 2-cm-radius beam currently used. When we compare them on the basis of equal radial forces, the corresponding radius of the 2000A beam is about $0.1 \mathrm{~cm}$. Also, the current-density distribution of this beam when ejected from the last cavity of PHERMEX would be such that most of the charge would lie within a couple of millimeters. In the absence of magnetic-confinement techniques, this constraint on beam radius $R$ is unique to highenergy injection. The permissible energy spread $\triangle V / V$ in the ejected PHERMEX beam of radius $R$ will vary as $t / 4 R$ (providing $t \leq 2 R$ ), where $t$ is the spot-size diameter. Thus, if $t \approx 2 R$, adequate focusing can, in principle, be accomplished when $\Delta \mathrm{V} / \mathrm{V}$ is as high as $50 \%$ (that is, most of the charge ejected from PHERMEX becomes accessible to a favorable spot size on the target).

In a standing-wave, linear-accelerator cavity, such as the preaccelerator or the PHERMEX cavities, the energy spectrum and convergence of the ejected electron beam depend on the injection phase angle as well as the cycle-to-cycle depletion of the electromagnetic energy stored in the accelerator caviiy. Energy depletion is, therefore, an important consideration in designing high-current accelerators.

We investigated energy depletion with the GRAPE SEED code. The total energy $(\Delta U)$ extracted from the cavity field per cycle was obtained by summing the energy extracted by all electrons injected during that cycle. The result, of this computational work for four cases are presented in Figs. 7 through 10, which show the fractional snergy depletion as a function of field strength in the cavity and the current injected over one cycle. Figure 7 illustrates the energy depletion for a 1-m preaccelerator cavity with a beam injection energy of $1.0 \mathrm{MeV}$. Figures 8 and 9 show the energy depletion in a $2.6-\mathrm{m}$ cavity for beams of 1.0 and $8.0 \mathrm{MeV}$, respectively. Figure 10 shows the energy depletion in a 2.6-m cavity when the output beam from the 1 $m$ preaccelerator is injected (Case 2).

The energy depletion calculated for the 1-m-long cavity represents no serious spread in the energy of a nominal 10-MieV beam injected into PHERIMEX, even for the 2000-A beam cases. The spread is even less offensive if the injected beam has been squeezed to a radius of a few millimeters. 


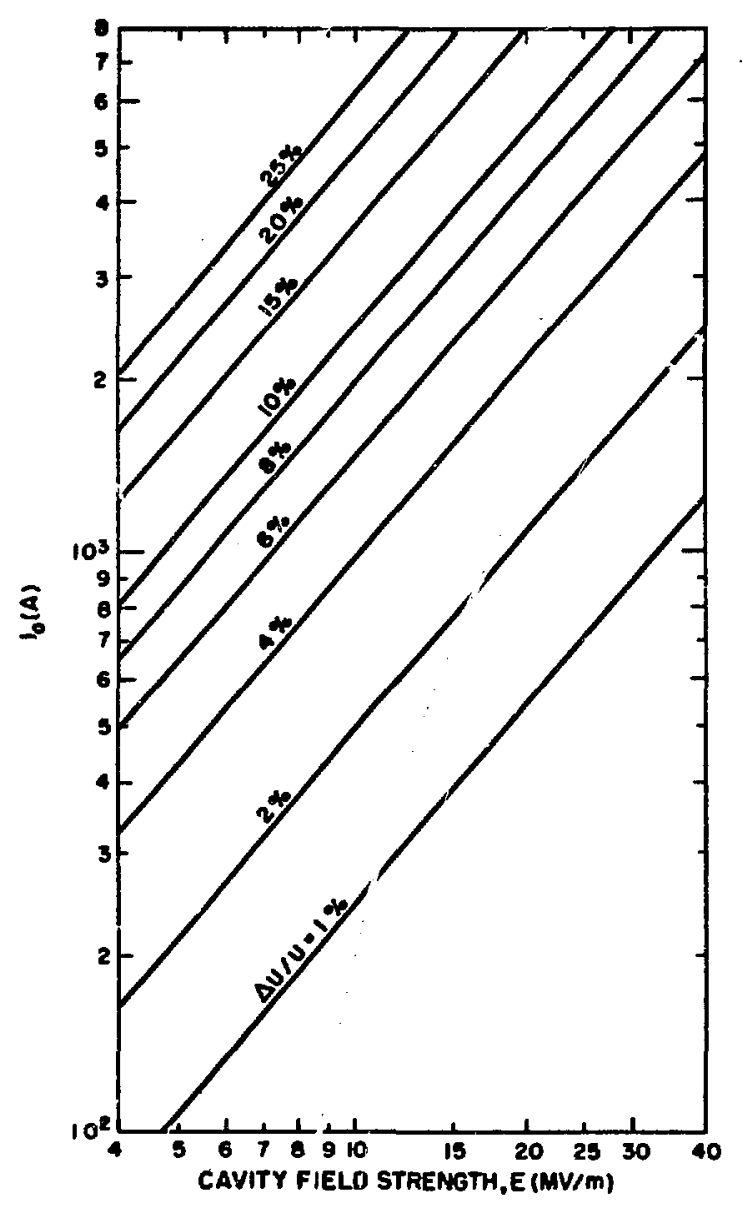

Fig. 7.

Energy depletion over one cycie for 1-m cavity and $\mathrm{V}_{0}=1.0 \mathrm{MeV}$.

Figures 11 and 12, for different beam currents injected into a $1-$ in cavity at 0.6 and $1.0 \mathrm{MeV}$, respectively, show the peak beam energy and the total charge within a radius of $5.0 \mathrm{~cm}$ that hits the plane of the downstream cavity wall at different cavity field strengths. The $5.0-\mathrm{cm}$ radius is approximately that of an exit aperture. For a given injection current, higher cavity field strengths cause more charge to be ejected within the $5.0-\mathrm{cm}$ radius. The higher cavlty. fleld strengths also produce beams that ere more tightly confined, an important feature not showi In these figures.

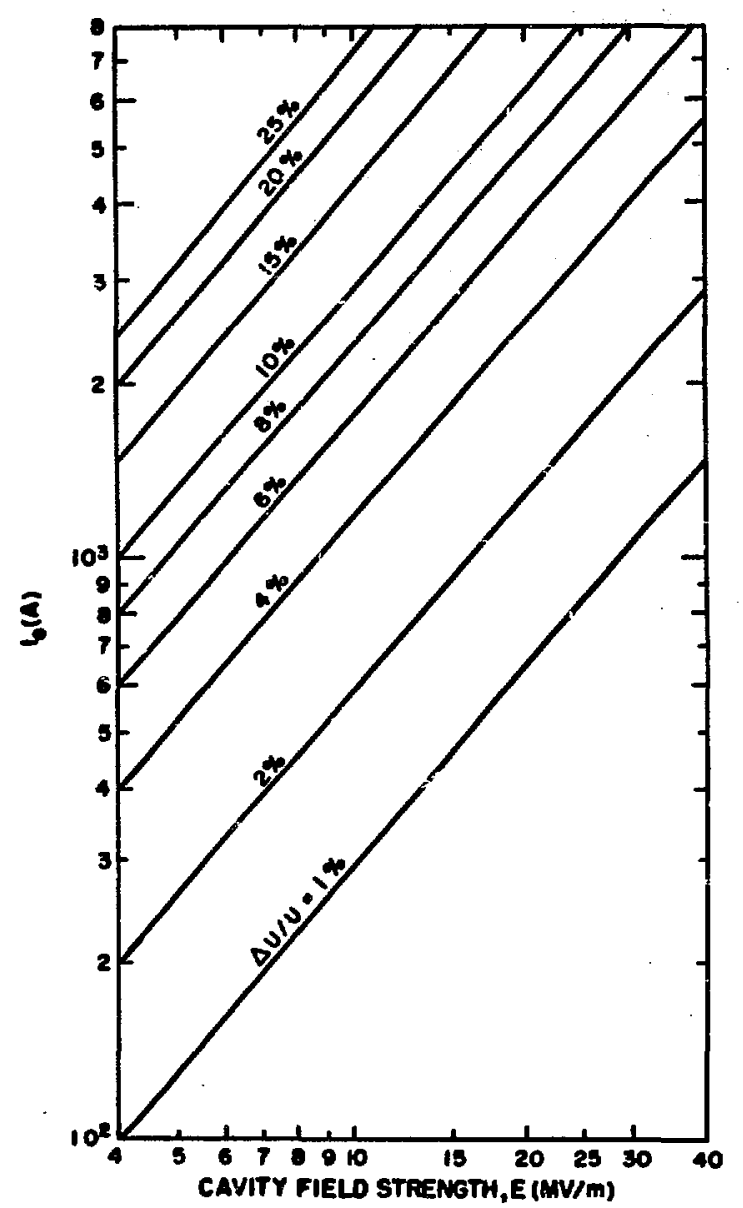

Fig. 8.

Energy depletion over one cycle for $2.6-m$ cavity and $V_{0}=1.0 \mathrm{MeV}$.

\section{CONClusions}

A nominal 0.5-MeV, 300-A, 2-cm-radius beam is not injected into PHEAMEX with zero convergence. The peak output-beam energy is about $30 \mathrm{MeV}$, and the typical flux of about 10 R per 5-ns burst corresponds to over $100 \mathrm{~A}$ for a 200-ns radiation pulse.

When upgraded, PHERMEX will have three 40ns electron bunches injected whose nominal beam parameters are $1 \mathrm{MeV}, 1000 \mathrm{~A}, 2-\mathrm{cm}$ radius. The expected peak beam energy is about $50 \mathrm{MeV}$, with a flux output of at least 100 R per $40-n s$ burst. 


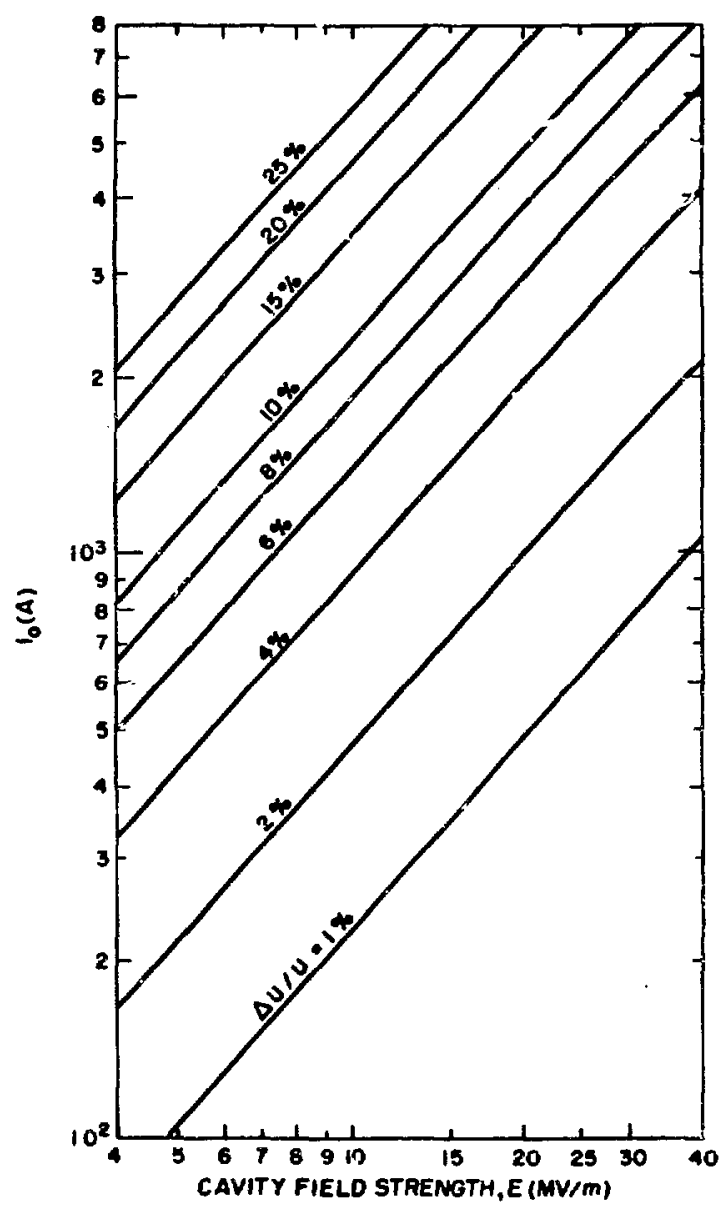

Fig. 9.

Energy depletion over one cycle for 2.6-m cavity and $V_{0}=8.0 \mathrm{MoV}$.

Correspondingly, the gains attributable to a preaccelerator (like that suggested in this report) represent substantial progress, even when injecting three sets of two 5-ns, 1000-A, 10-MeV, 1$\mathrm{mm}$-radius bunches of electrons. The radiation per

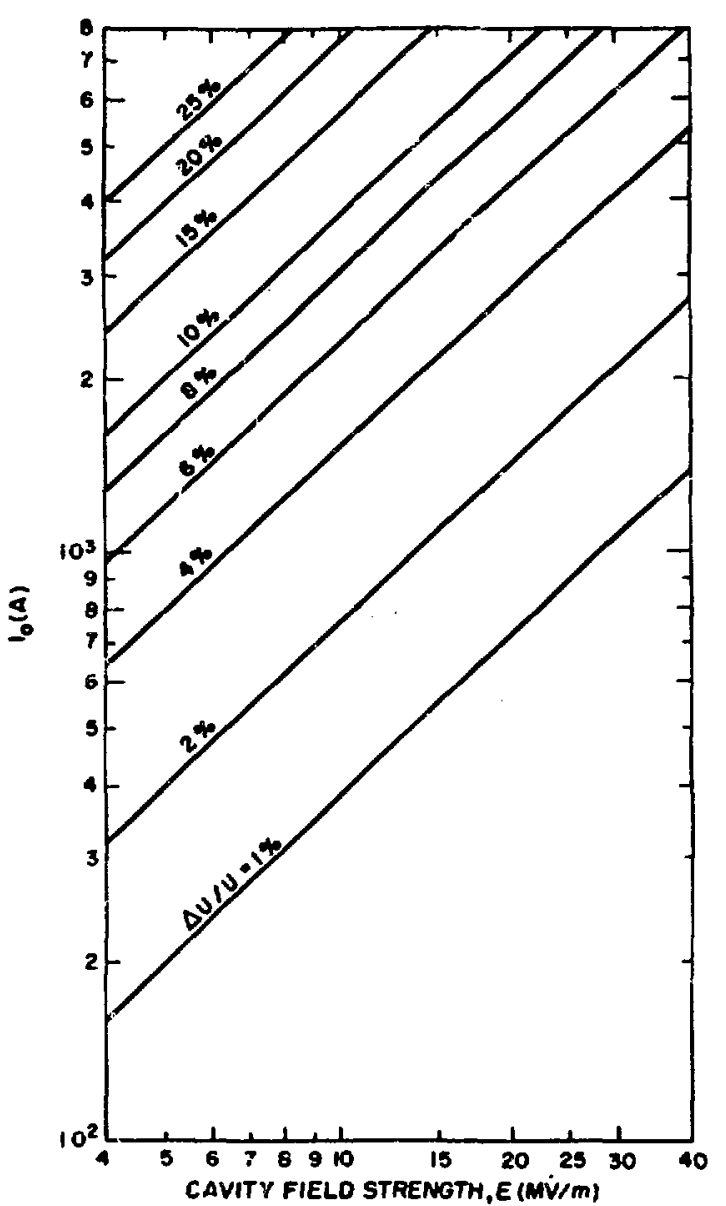

Cin. 10.

Energy depletion in $8.6-\mathrm{m}$ cavity for output beam injected from 1-m cavity where $E=10$ $M V / m$ and $V_{0}=1.0 \mathrm{MeV}$.

40-ns pulse (two 5-ns bunches) would be at least $200 \mathrm{R}$.

Table II compares these various real and projected PHERMEX stages. 


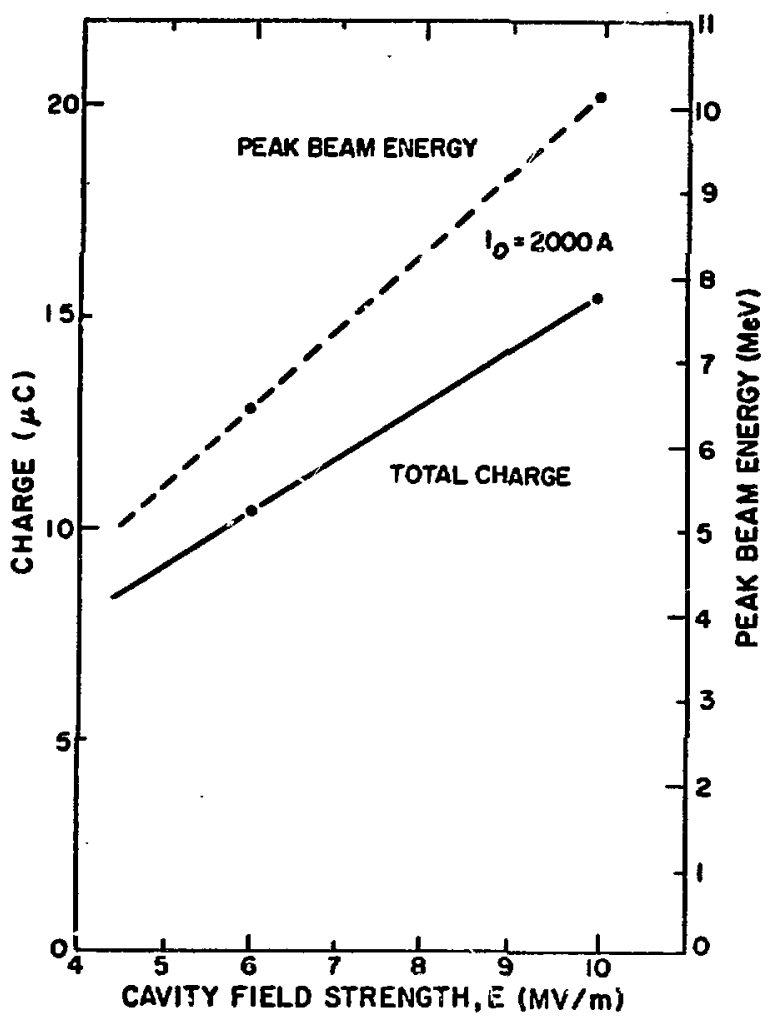

Fig. 11.

Peak besm energy and total charge that hits downstream wall of $1-m$ cavity within $5.0-\mathrm{cm}$ radlus vs cavity field strength; $v_{0}=0.6 \mathrm{sioV}$.

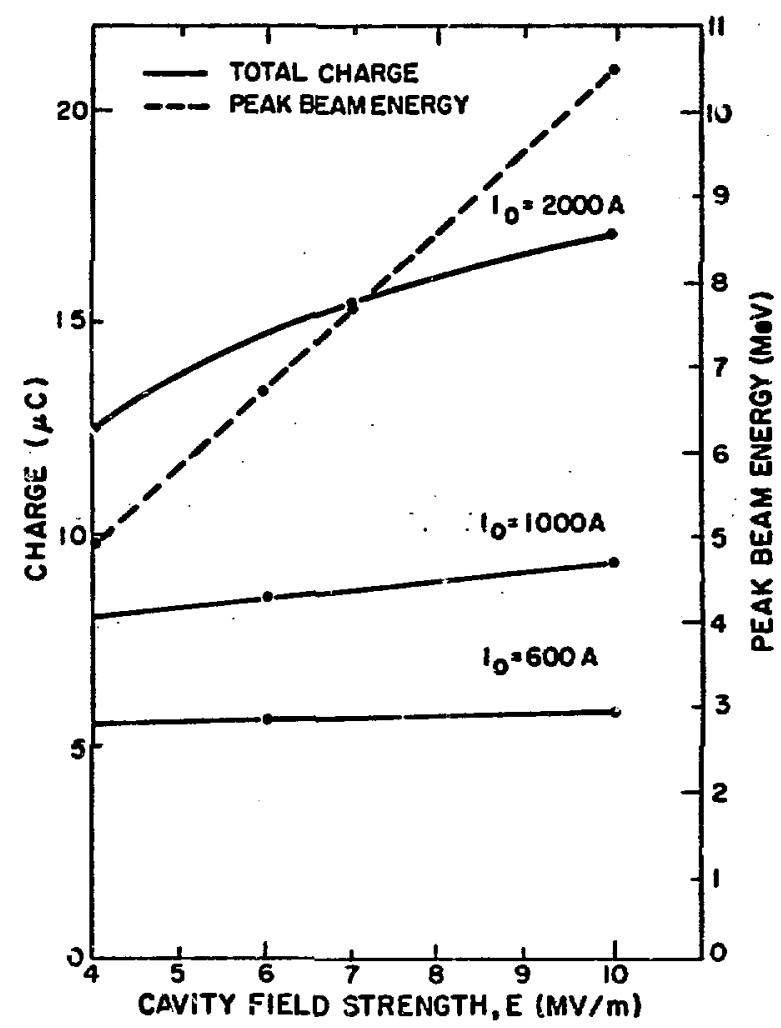

Fig. 12.

Peak beam energy and total charge that hits downstream wall of $1-\mathrm{m}$ cavity within $5.0-\mathrm{cm}$ redius vs cavity fielof strongth; $v_{0}=1.0 \mathrm{MeV}$.

TABLE II

PHERMEX PARAMETERS

\section{Present Upgraded Presccelerctor}

$\begin{array}{lccc}V_{o}(\mathrm{MeV}) & 0.5 & 1 & 10 \\ \mathrm{I}_{0}(\mathrm{~A}) & 300 & 1000 & 1000 \\ \mathrm{P}_{0}(\mathrm{~cm}) & 2 & 2 & 0.1 \\ \text { Pulses } & 1(200 \mathrm{~ns}) & 3(40-\mathrm{ns}) & 3(40-\mathrm{ns}) \\ \mathrm{V}(\mathrm{MeV}) & 30 & \sim 50 & \sim 57 \\ \text { Radiation (R) } & 100+ & 300-500 & \sim 1000 \\ \text { Radiation (R)/ } & 10+ & 30-50 & \sim 100 \\ \text { 5-ns subburst } & & & \end{array}$




\section{REFEйtences}

1. Douglas Venable, D. O. Dickman, J. N. Hardwick, E. D. Bush, Jr., R. W. Taylor, J. J. Boyd, J. R. Ruhe, E. J. Schneider, B. T. Rogers, and H. G. Worstell, "PHERMEX: A Pulsed HighEnergy Radiographic Machine Emitting $X$ -
Fiays," Los Alamos Scientific Laboratory report LA-3241 (1967).

2. T. H. Martin, "Determination of Bremsstrahlung Production Efficiencies from Data Obtained on PHERMEX at 27 MeV," Sandia Laboratories report SC.-DA-69-240 (1969).

\section{APPENDIX A}

\section{FIGURES OF MERIT FOR RADIOGRAPHIC EXPEAIMENTS}

Two ciistinctly different radiographic experiments had to be considered when we designed PHERMEX. One class of experiments demanded peak radiation flux per unit area distributed over a region significantly smaller than the radiographic object, whereas the second class required a high average flux coinpleteiy irradiating the region of interest.

In experiments of the first class, we obtain high intensities by simply increasing the beam energy, which both. narrows the radiation cone and increases the radiation production efficiency. Indeed this technique is one of the primary selling points for present-day traveling-wave electron accelerators as radiation generators. In this class of experiments a figure of merit for relative output intensities of $\mathrm{X}$-ray machines can be defined as the ratio of beam current at an arbitrarily chosen standard energy to the beam current at some other energy that will produce the same useful axial radiation intensity. Such a relative figure of merit (Fig. A-1) has been determined as a function of energy for the two radiographic extremes: skiagraphy, wherein only shadow profiles are obtained, and densitometry. We used a standard energy of $20 \mathrm{MeV}$, which requires 9.3 times the current of a $5 n-M e V$ beam to be equally useful for skiagraphic service, and it requires 3.5 times as much to provide the same useful transmitted radiation intensity when radiographically penetrating 10 $\mathrm{cm}$ of uranium. High electron-beam energies are therefore important for this class of radiography.

We define another important figure of merit for the second class of radiographic experiments, where total useful flux transmitted through the experimental object is our criterion rather than the relative intensity. This figure of merit is paramount when radiographing a thick object of fixed size. The energy adyantage is much less important because it is the production efficiency of 3- to 4-MeV quanta

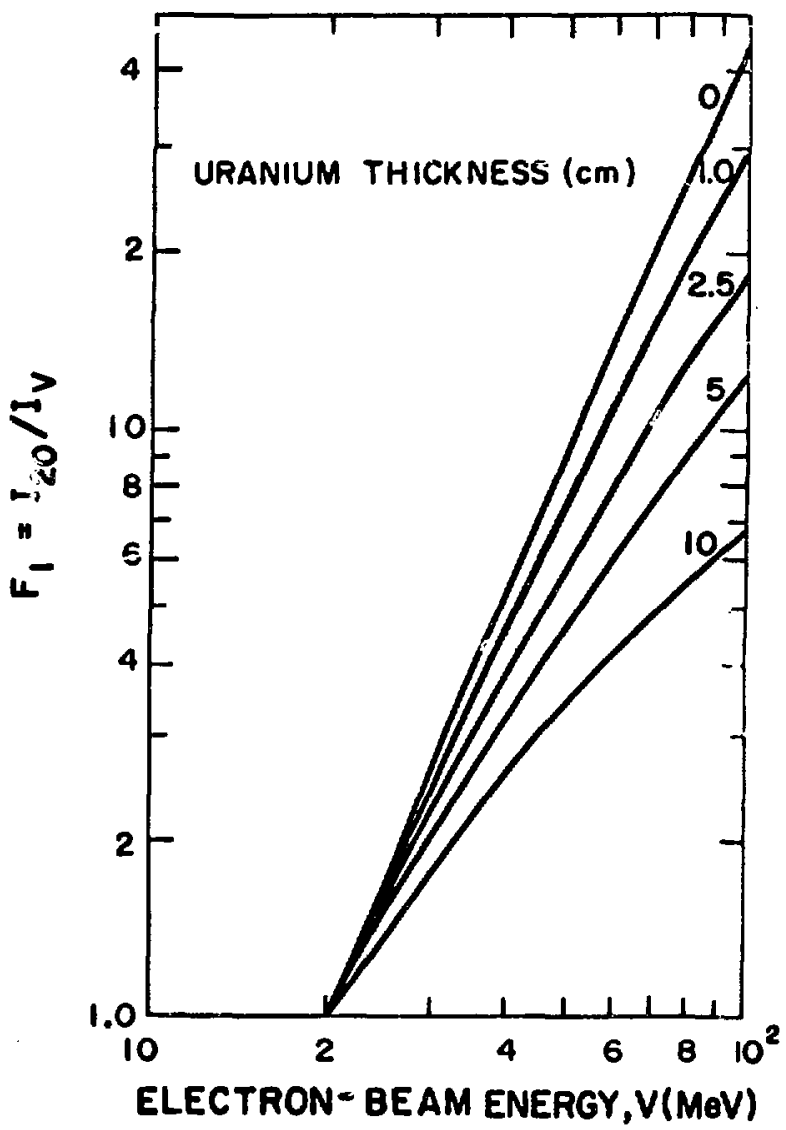

Fig. A-1.

Figure of merlt for radlographic machines where maximum useful radiation intensity is demanded. 
that is significant in whole-object panetration experiments with uranium, as seen in Figs. A-1 and A-2. Again, in examining the radiation generated by $20-$ and $50-\mathrm{MeV}$ electron beams, we find that a $20-\mathrm{MeV}$ beam must have $50 \%$ more current than a 50-MeV beam to generate the same skiagraphically useful radiation flux. On the other hand, for radiographic penetration of $10 \mathrm{~cm}$ of uranium, the 50-MeV beam must hav 7 about $80 \%$ 'more current than the 20-MeV beam. ' 10 higher energy beams offer no obvious advantage to whole-object radiodensitometry for peinetrating thick sections of uranium; the rea! yains in radiation intensity are obtained by increasing the electron-beam curient.

Vory few experiments in current radiography require a maximum of radiation energy. Most experiments depend on on-uxis intensity for success, and Fig. A- 1 is thus more appropriate.

In the present PHERMEX-Enhancement Progiam, greater radiation energy and radiation intensity are primary objectives. Radiation gnergy increases with the charge delivered to the target and with the bremsstrahlung production efficiency. Radiation intensity increases with the charge delivered to the target and with the bremsstrahlung production efficiency and varies inversely with the radiation cone angle. Deliverable charge varies with cavity field strength; production efficiency, with the 0.8 power of ejected-beam energy; and cone angle, inversely with the square of beam energy. The ejected-beam energy is roughly the sum of the injected-beam energy and the energy gain provided by the cavities, which varies with the field strength. As a very rough rule of thumb, the on-axis radiation intensity can vary with the 3.8

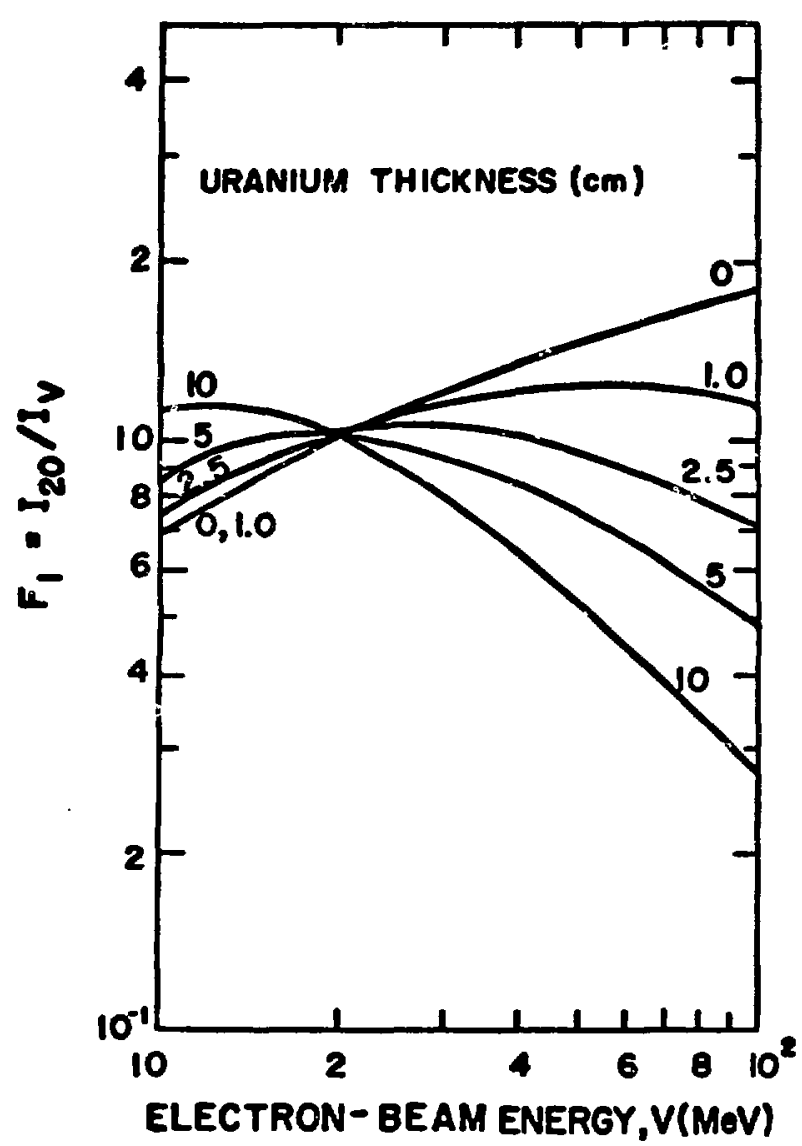

Fig. A-2.

Figure of merit for radiographic machines where maximum useful radiation flux is required to penetrate uranic $n$.

power of the cavity fields, provided the charge injected is consistent with the maximum acceptaole by the first accelerating cavity.

\section{APPENDIX $\mathrm{B}$}

\section{DETAILED COMPUTATIONS AND GRAPE SEED MODIFICATONS FOR A PREACCELERATOR-PREBUNCHING TECHNIQUE FOR PKERMEX}

\section{INTAODUCTION}

This appendix presents the detailed computations on which we based our conclusions about the utility of a $1-\mathrm{m}$-long, $50-\mathrm{MHz}$, preaccelerator bunching cavity for PHERMEX. Included are the GRAPE SEED code modifications implemented to study the variety of problems.

PHERMEX is designed to provide a highintensity pulse of bremsstrahlung having adequate 
flux with suitable distribution of quantum energy appropriately directed to satisfy certain flashradiography requirements. We considered an existing electron-gun design whose perveance was about $10^{-6}$ and which would produce about $1000 \mathrm{~A}$ at about $1 \mathrm{MeV}$. The gun could bepulsed with stateof-the-art pulsers to produce a programmed series of 40-ns-long pulses. We assumed that this gun, coupled with state-of-the-art magnetic lenses, could inject a monoenergetic electron beam of radius $A_{0}$ and uniform convergence into the preaccelerator. A block diagram in Fig. 8-1 illustrates the arrangements.

For a $50-\mathrm{MHz}$ preaccelerator, the maximum charge in a 40-ns output-beam pulse is determined by the charge that can be concentrated into two rf cycles. (For simplicity, we considered one if cycle only; considerations for the second cycle are the same as for the first except for the interaction caused by the beam, nergy depletion of the first cycle. For the beams studied here, we also ignored the effect of energy depletion on electrons within a cycle.) This charge is limited by characteristics of the electron gun and pulser, the design of which is partly determined by low-jitter and beam-optics considerations. Gun jitter, while important in experiments, was unimportant to our calculations and will be mentioned no further.

For given beam and cavity parameters, we did a preliminary GRAPE SEED study to determine the stable electron orbits (those terminating at the plane of the downstream wall of the preaccelerator cavity regardless of the radius, energy, and con- vergence of the electron beam there) as a function of injection phase angle or injection time. The computations then carried the stable-orbit beam electrons from injection into the preaccelerator to the downstream wall and through a field-free 1-mlong drift space where the beam was allowed to expand radially as a result of space charge. The change in beam quality as it traversed the drift space was then a measure of the need to consider beam optics there.

The function of the preaccelerator we studied was to increase the number of particles within acceptable ranges in phase space. We were thus interested in the quality of the boam (charge-density distribution, compactness, energy distribution, and convergence) that could be injected into PHERMEX from the sreaccelerator. This beam quality necessarily depends on parameters of the injected beam (radius, current, energy, and convergence) and preaccelerator-cavity field strength, and will eventually determine the flux produced at the target. Table $I$ in the main text lists the beam and preaccelerator-cavity parameters we studied. For comparison, we studied some beams injected directly into a regular 2.6-m-long PHERMEX cavity. In addition, we ran one beam through a two-cavity tandem arrangement in which a beam was injected into a 1-m-long cavity and then drifted $1 \mathrm{~m}$ before it was injected into a 2.6-m-long cavity.

We emphasize that no attempt was made in this report to optimize any parameters for subsequent design purposes, and no cost-cffectiveness arguments were considered.

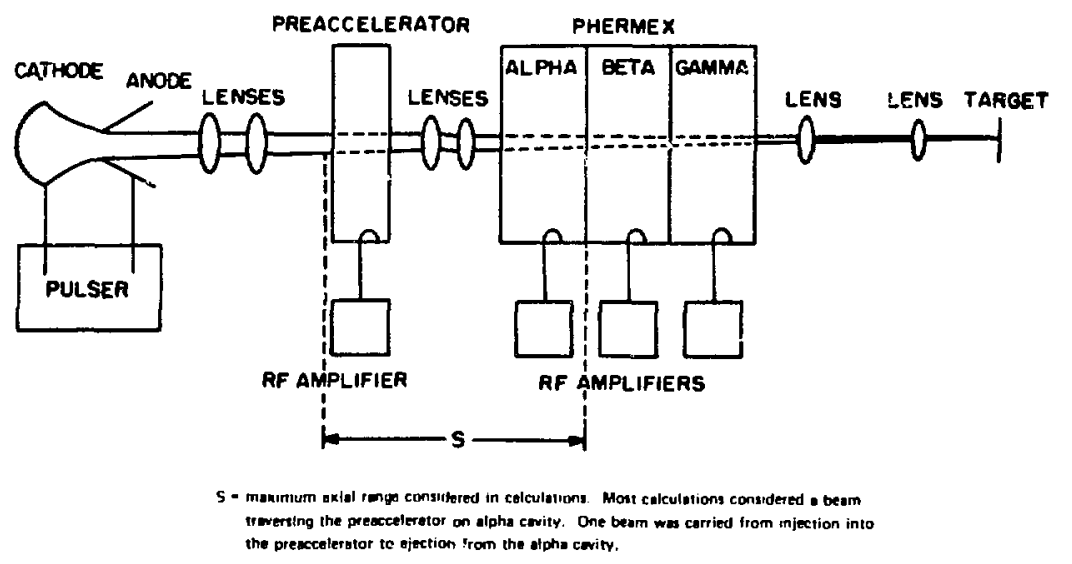

Fig. B-1.

Block diagram of experimental setup. 


\section{GRAPE SEED MODIFICATION}

D. O. Dickman described the principal features of GRAPE SEED in LASL report LA-3241. For our work, the GRAPE SEED code was substantially modified to accommodate the variety of problems of interest.

Assime that a cylinder of current $I_{0}$ and radius $A_{0}$, with its length much greater than its radius, is injected with electrons at energy $V_{o}$. The code considers a representative peripheral particle having en arbitrary injection phase angle, follows its path through a given accelerator cavity, and computes the attendant fields at each point. Provisions are made for aperture corrections and various instability tests.

The entrance and exit apertures act as two lenses. Depending on the direction of the electric field of the cavity and, hence, the injection phase angle, an electron receives a positive or negative contribution to its radial mo nentum as a result of the aperture effect. In our cilculations, we applied the aperture effect to the electron in the plane of each aperture.

To compute the current densily at ine and of a cavity when a uniform-current-density beam of radius $A_{0}$ and energy $V_{0}$ is injected, the input data for GRAPE SEED divide the injected beam into cylinders of uniform current density with radii $A_{1}$ (= $\left.A_{0}\right) A_{2} \ldots A_{n}$ enclosing currents $I_{1}\left(=I_{0}\right) I_{2} \ldots I_{n}$, where $\mathrm{n}$ is typically 10 to 13 . The acceptance phase-angle window (the interval over $360^{\circ}$ of injection phase angles $\Phi$ having stable orbits) was about $185^{\circ}$ for the beam and cavity parameters. This acceptance window was sampled at $5^{\circ}$ intervals. For each set $\left(I_{n}, A_{n}, V_{0}\right)$, there were about 38 phase angles and about 494 GRAPE SEED problems that were run for a given beam and given cavity parameters.

Two subroutines, NEWSUB and CURDEN, were added to the modified GRAPE SEED code, and a program SIGSUM was written to obtain the data required in this study.

\section{A. NEWSUB}

A Runge-Kutta integration routine, which determines the time interval best suited to the problem for the accuracy specified, is used in GRAPE SEED to integrate the equations of motion in a cylindricalcoordinate system. As the equations of motion are integrated for a given set of $\phi$ and $V_{0}$ and initial $I_{0}$ and $A_{n}$, the quantities $T$ (time), $Z$ (axial posit:on), $R$ (radius), $\theta$ (angular position), $\dot{Z}, \dot{R}, \dot{\theta}$, and $V$ (energy) are computed and stored. NEWSUB uses a spline function routine to interpolate these quantities at $Z_{1}$ and $Z_{2}$, the end of the cavity and drift tube, respectively. $\left(Z_{1}=1.0 \mathrm{~m}\right.$ and $Z_{2}=2.0 \mathrm{~m}$ for the 1-m-long preaccelerator plus $1-\mathrm{m}$-long drift space; $Z_{1}=2.6 \mathrm{~m}$ and $Z_{2}=3.2 \mathrm{~m}$ for the case where a regular PHERMEX cavity and $0.6-\mathrm{m}$ drift space are of interest.) These quantities are then stored. From the interpolated values of $\dot{R}, \dot{Z}$, and $R$ at $Z_{1}$ and $Z_{2}$, NEWSUB calculates and stores the convergence $C$, defined as $C=-(\dot{R} / Z) / R$.

These quantities are then printed. Typical outputs are shown in Figures B-2 and B-3.

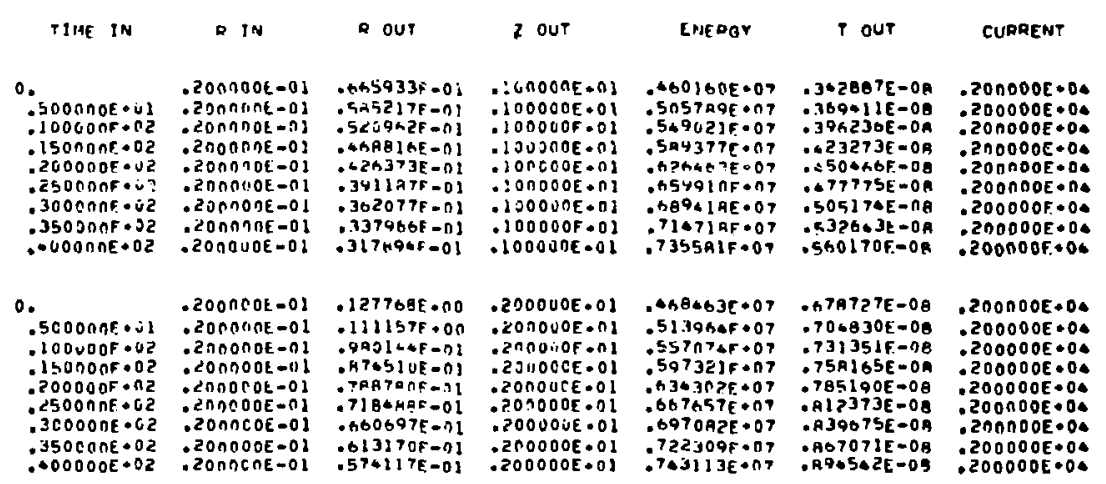

Fig. B-2.

Typical output trom NEWSUB. 


\begin{tabular}{|c|c|c|c|c|c|c|c|}
\hline TIME IN & 2 & THETA & R-UOT & Z-00T & THE TA-DOT & $n p / 02$ & CONVERGEMCE \\
\hline 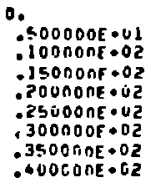 & 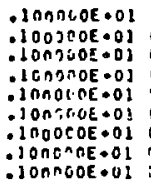 & $\begin{array}{l}n: \\
i: \\
n: \\
i: \\
0: \\
i: \\
i:\end{array}$ & 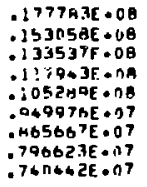 & 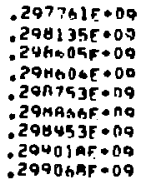 & $\begin{array}{l}0 . \\
0 . \\
0 . \\
0 . \\
0 . \\
0 . \\
0 . \\
0 . \\
0 .\end{array}$ & 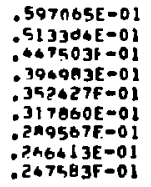 & 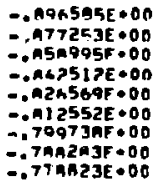 \\
\hline 0. & $.2000 S 0 E \cdot 01$ & 0. & - IASBITE OB & $.2077 n 1 E \cdot n 9$ & 0. & $.62337 .0-01$ & $\because \triangle A>B 9 B E+00$ \\
\hline $\begin{array}{l}.500000 E+01 \\
.100000 E 0.02 \\
.15000 \text { OE } 62\end{array}$ & 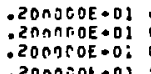 & j.: & $\begin{array}{l}.1611001 E+A 8 \\
.139409 E \bullet 08 \\
.123709 E+0 B\end{array}$ & 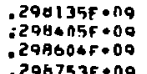 & $\begin{array}{l}0 . \\
0 . \\
0 .\end{array}$ & $\begin{array}{l}\text { 53A674E-01 } \\
\because 4+4522 E-01 \\
\because 14 P 92 E-01\end{array}$ & 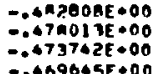 \\
\hline $\begin{array}{l}-200000 E+62 \\
-250000 E=02 \\
.300000 E+02\end{array}$ & 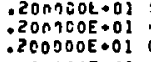 & $\begin{array}{l}3 . \\
0 .\end{array}$ & $\begin{array}{l}110672 E * 08 \\
1106096 E-0 B \\
.9166 B 2 E \rightarrow 07\end{array}$ & $\begin{array}{l}.206753 E+09 \\
.29 H A B A E+00 \\
.29895 .1 E+119\end{array}$ & $\begin{array}{l}0 . \\
0 . \\
0 .\end{array}$ & $\begin{array}{l}.370646 E-01 \\
334930 E=01 \\
.375956 E-01\end{array}$ & $\begin{array}{l}=\triangle A 9605 E+00 \\
=\triangle A R 159 E * B C \\
=\triangle A 30 C D E+00\end{array}$ \\
\hline $\begin{array}{l}.350 \text { T506.0द } \\
.400000 E-02\end{array}$ & $\begin{array}{l}-\angle 00 n c 0 E-01 \\
2 \operatorname{2nOnOOE} 001\end{array}$ & ".. & 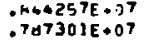 & $\begin{array}{l}.29901 A F+09 \\
.29906 A E+0 Q\end{array}$ & 0. & $\begin{array}{l}.2 A 2343 F-01 \\
.263252 E-01\end{array}$ & $\begin{array}{l}\because 4 h n 465 E \bullet 0 n \\
\because 65 n 533 E \bullet 00\end{array}$ \\
\hline
\end{tabular}

Fig. B-3.

Typical output from NEWSUB.

\section{B. CURDEN}

Subroutine CURDEN computes : $1 e$ contribution of current density $\sigma$ (referred to as SIGI in CURDEN) for a given $\phi$ and $Z_{1}$ (or $Z_{2}$ ). We obtain $\sigma$ by the relation

$\sigma_{n}=\frac{\left(I_{n}-I_{n+1}\right)}{\pi\left(R_{n}^{2}-R_{n+1}^{2}\right)}$

where $R_{n}$ and $R_{n+1}$ are the radii at $Z_{1}$ (or $Z_{2}$ ) and were initially $A_{n}$ and $A_{n+1}$, respectively. ( $A$ and $R$ are called RO and ROUT, respectively, in CURDEN.)
For a given $\Phi$ and $Z_{1}$ (or $Z_{2}$ ), the quantities $A, R$, $V, T, \sigma, R, \dot{Z}, \dot{\theta}, \dot{R} / \dot{Z}$, and $C$ are printed. Figure $B-4$ shows a typical output. CURDEN also punches $\left(R_{n}\right.$, $\sigma_{n}$ ) for a given $\sigma, Z_{1}$ and $Z_{2}$. A program called SIGSUM then uses these data.

\section{SIGSUM}

For a given $Z_{1}$ and $\left(Z_{2}\right)$, the SIGSUM program takes the current-density-distribution $\left(R_{n}, \sigma_{n}\right)$ output from CURDEN for all the injection phase angles (in $5^{\circ}$ intervals) within the acceptance window and

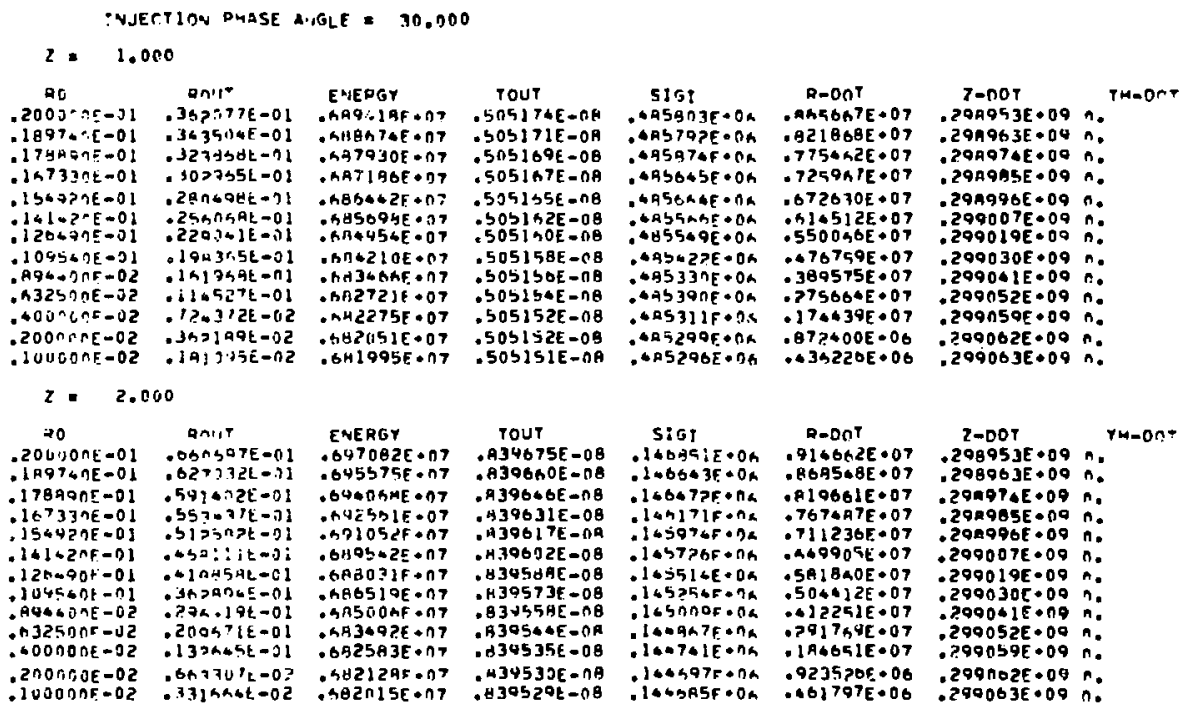

Flg. B-4.

Typical output from CURDEN. 
computes and prints the charge-density distribution, the integral of charge density vs radius, and the scaled integral of charge density vs radius (the fraction of total charge within radius $R$ vs $R$ ). The total or net charge-density distribution and the scaled integral vs radius are also plotted on film.

Briefly, SIGSUM proceeds as follows. Calling $\left(R_{n}, \sigma_{n}\right)$ for a given $\Phi$ set of data, the sets of data for all acceptable $\Phi^{\prime} s$ are read in and scaied by $\left(5^{\circ} / 360^{\circ}\right) \times 20 \mathrm{~ns}$. The sets of data are thus converted to sets of charge-Jensity distributions. The individual sets are then fit with spline functions and the charge-density contributions for each set are summed to yield the total charge-density distribution, which is then integrated and scaled.

\section{RESULTS}

Figures B-5 through B-134 display the results of this computational study graphically. Figures B-5 through B-114 apply to studies of a 1-m-long cavity, Figures B-115 through B-129 to a $2.6-\mathrm{m}$ long cavity, and Figs. B-130 through B-134 to a tandem arrangement where the output of a $1-\mathrm{m}$ long cavity is injected into a $2.6-\mathrm{m}$-long cavity. We considered no externally imposed magnetic fields except for the two-cavity-tandem study, for which our computations assumed that a magnetic fielo of appropriate distribution in the $1-\mathrm{m}$ drift space between cavities kept $R$ equal to 0 . The computations explored variations in the beam parameters of injected beam current, energy, and convergence and in cavity field strength. The initial beam radius was held constant at $2.0 \mathrm{~cm}$ at all times. Each graph shows the pertinent variables. For given beam and cavity parameter, some characteristics of the resulting beam eiected from the appropriate cavity and drift space are illustrated in successive sets of five figures each beginning with Figs. B-5 (chargedensity distribution), B-6 (fraction of total charge nitting downstream wall with radius $\mathrm{A}$ ), B-7 (energy of ejected beam vs injection phase angle), B-8 (convergence of ejected beam vs injection phase angle), and B-9 (energy of ejected beam vs radius).

Increased injection energy, higher field strength, or both will produce a tighter beam and, of course, a higher peak beam energy. Another benefit of higher field strengths is less energy depletion for a given injected beam. Variations in injection convergence $\left(C_{o}\right)$ indicate that small, positive, initial convergences can result in tighter beams. The exit convergence spectrum is also affected and could influence beam optics for the ejected beam. 


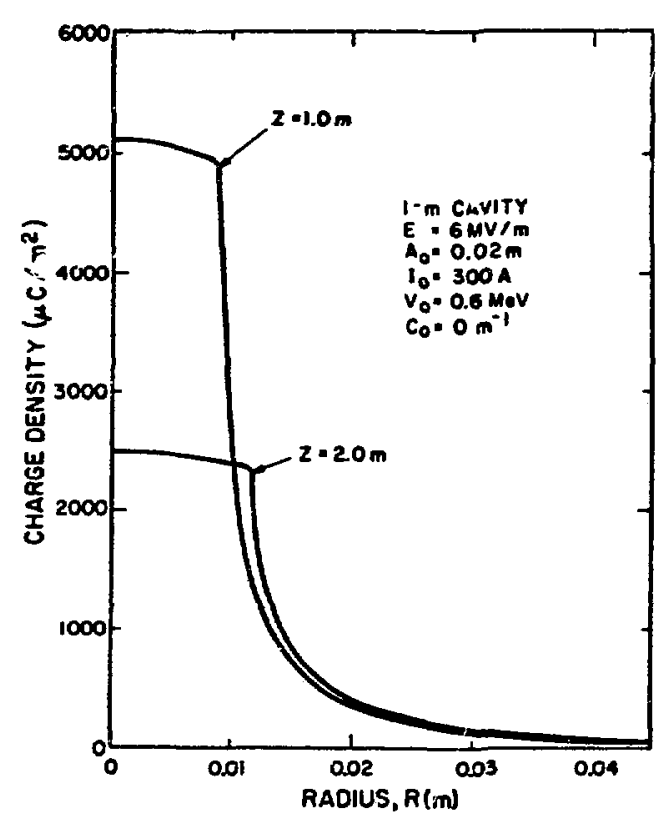

Fig. B-5.

Charge density vs radius.

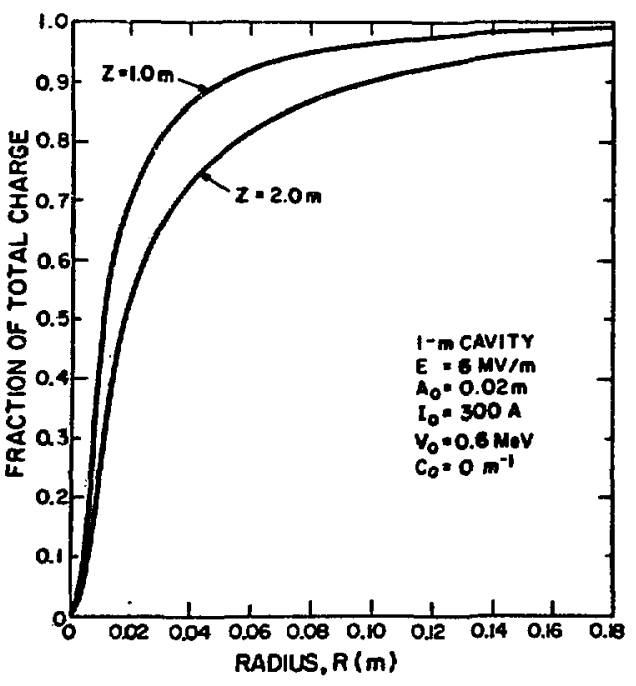

Fig. B-6.

Fraction of total charge hitting downstream wall within radius $R$.

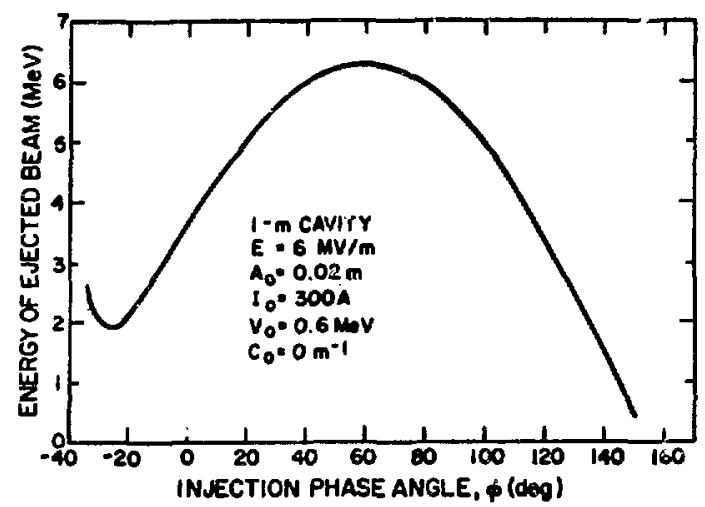

Fig. B-7.

Energy of ejected beam vs injection pinase angle.

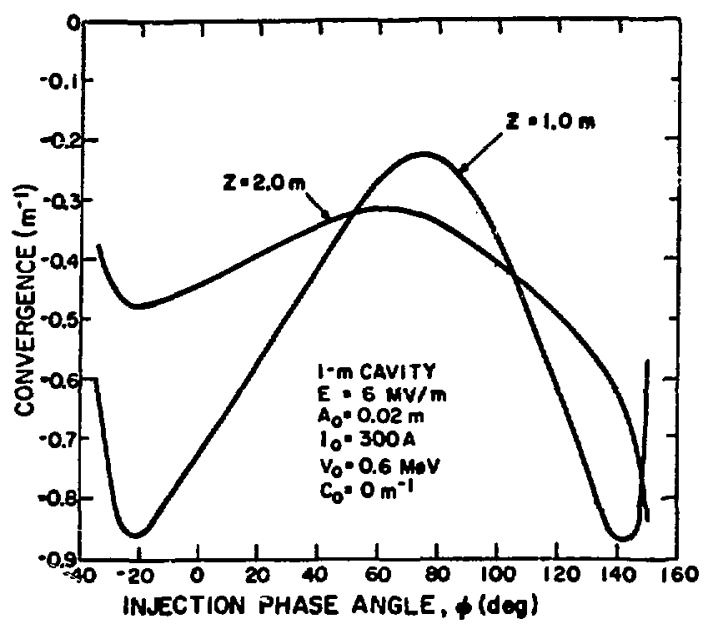

Fig. B-8.

Convergence of ejected beam vs injection phase angle.

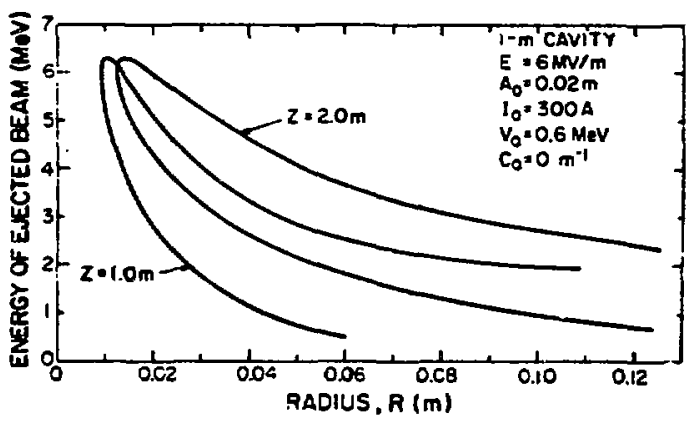

Fig. B-9.

Energy of ejected beam vs radius. 


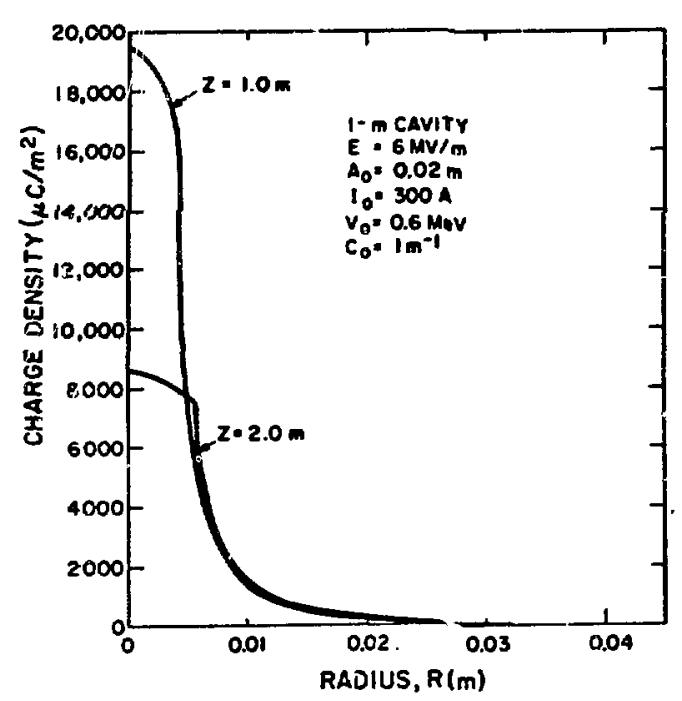

Fig. B-10.

Charge density vs radius.

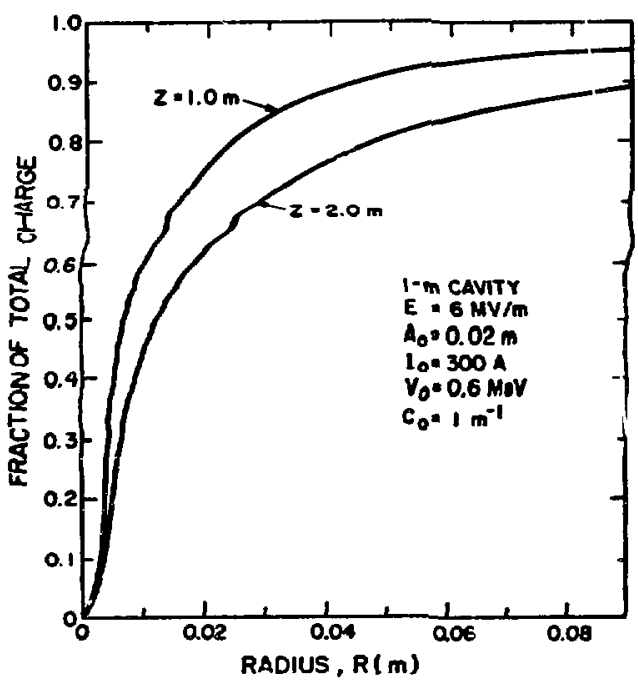

Fig. 8-11.

Fraction of total charge hitting downstream wall within radlus $R$.

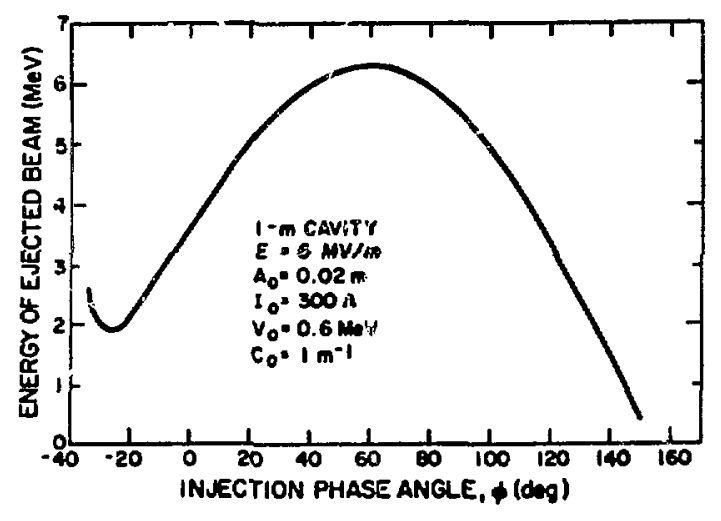

Fig. B-12.

Energy of ejected beam vs injection phase angle.

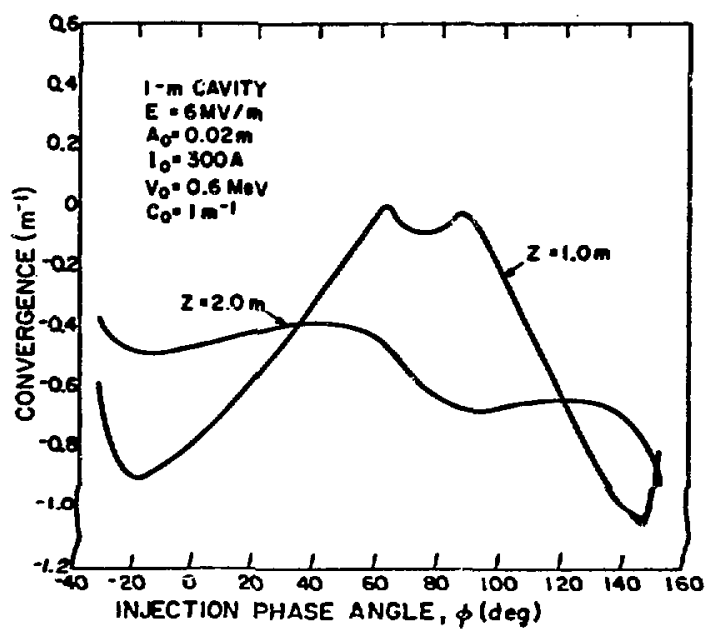

Fig. B-13.

Convergence of ejected beam vs injection phase angle.

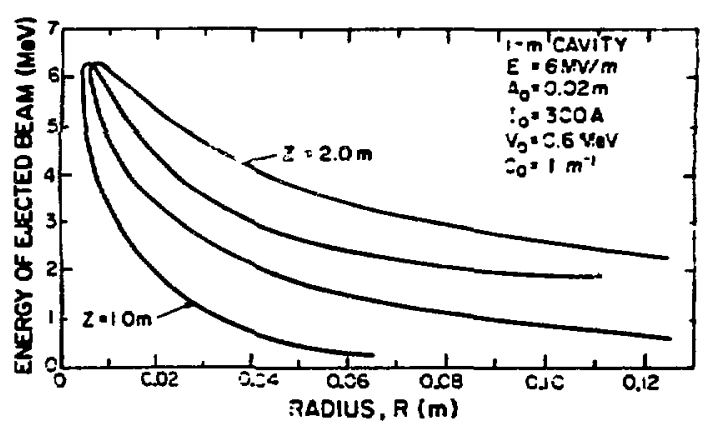

Fig. $B-14$.

Energy of ejected beam vs radius. 


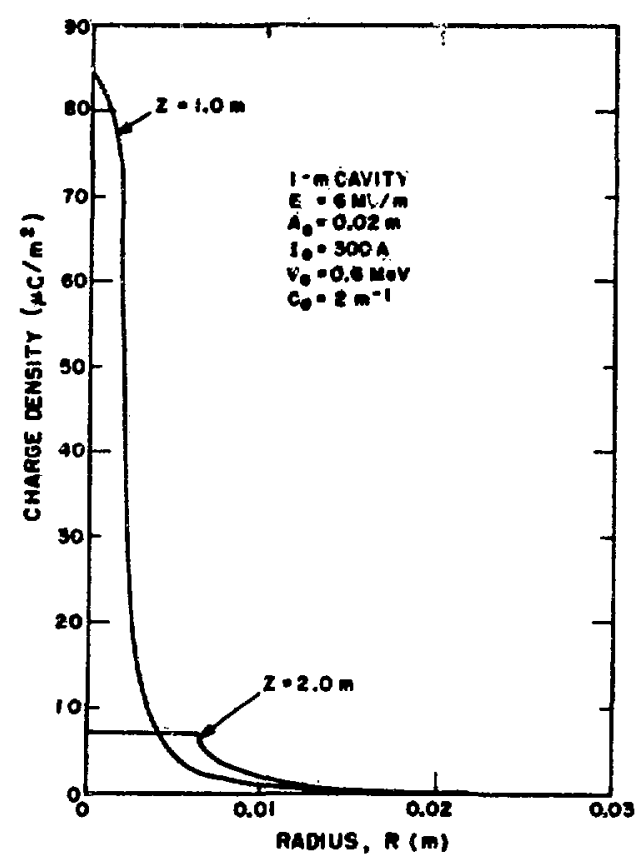

Fig. B-15.

Charge density vs radius.

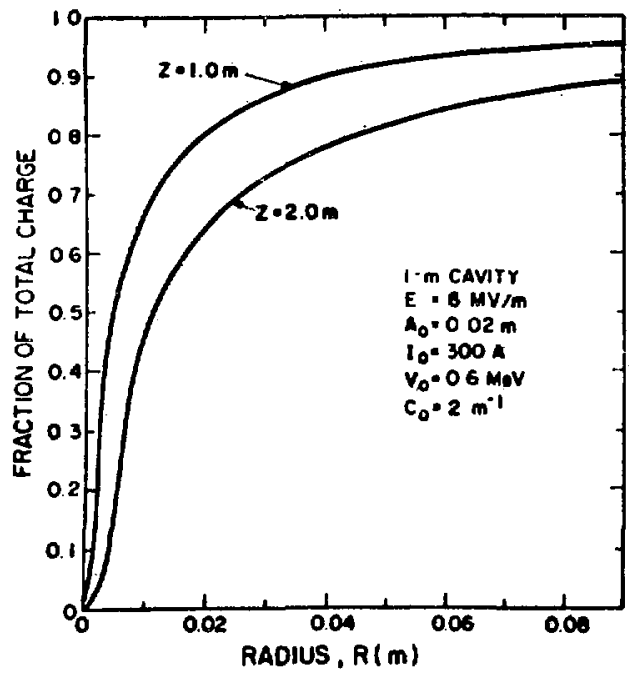

Fig. B-16.

Fraction of total charge hitting downstream wall within radius $R$.

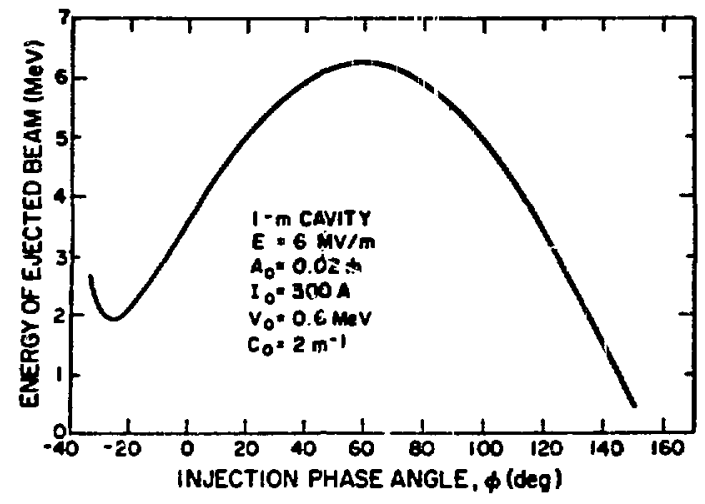

Fig. B-17.

Energy of ejected beam vs injection phase angle.

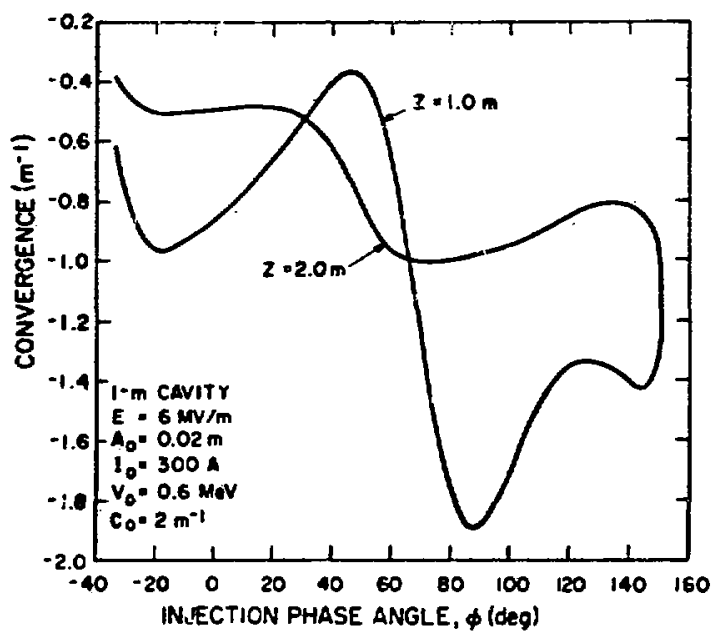

Fig. B-18.

Convergence of ejected beam vs injection phase angle.

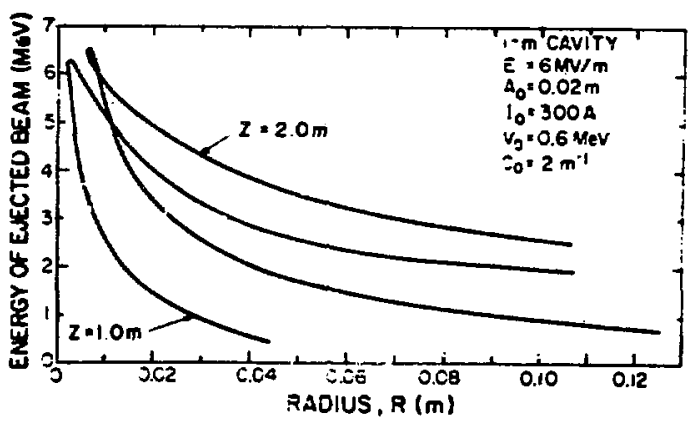

Fig. B-19.

Energy of ejected beam vs radius. 


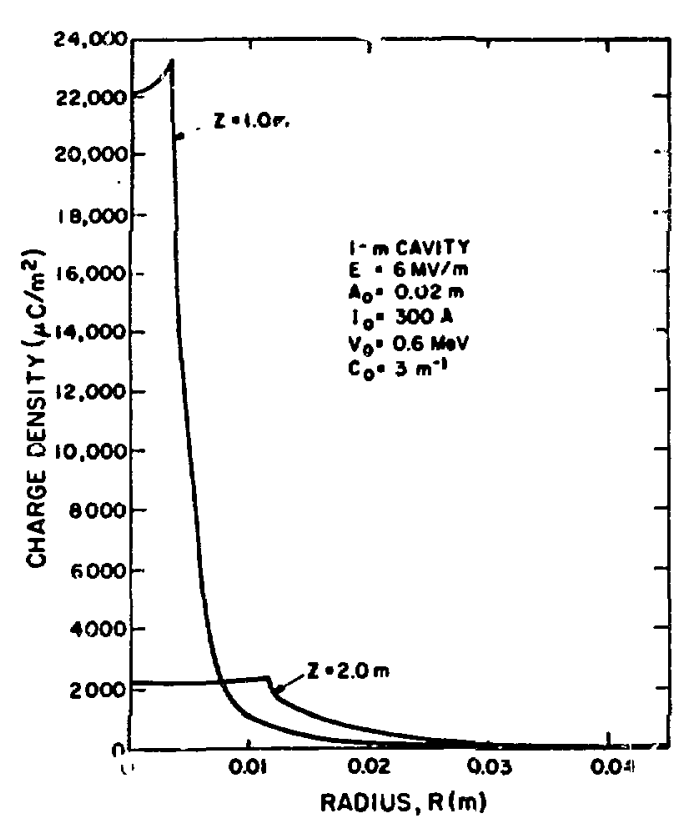

Fig. B-20.

Charge density vs radius.

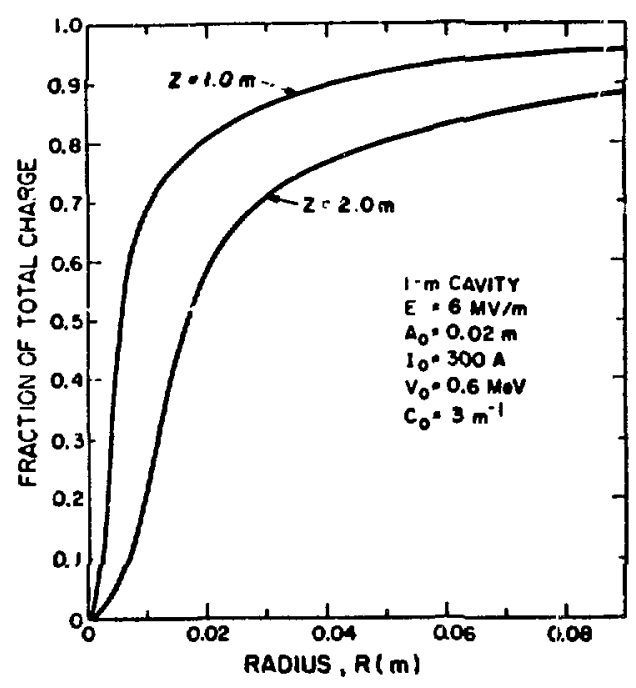

Fig. B-21.

Fraction of total charge hitting downstream wall within radius $R$.

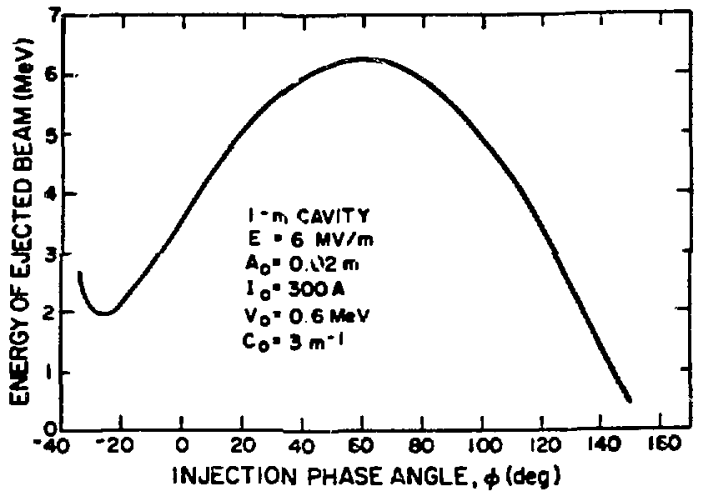

Fig. B-22.

Energy of ejected bi:m vs injection phase angle.

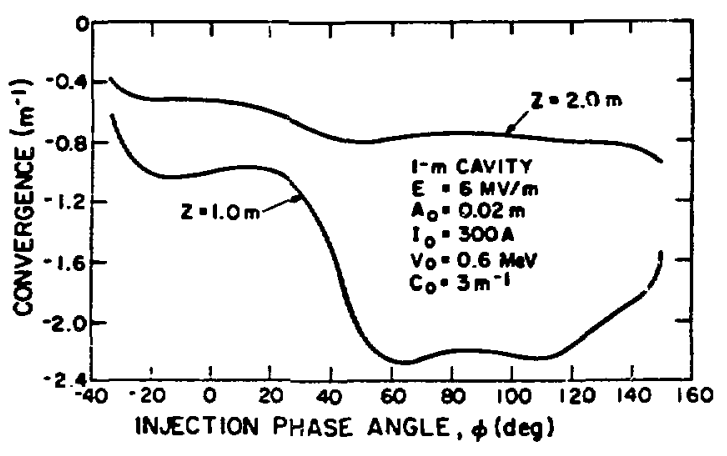

Fig. B-23.

Convergence of ejected beam vs injection phase angle.

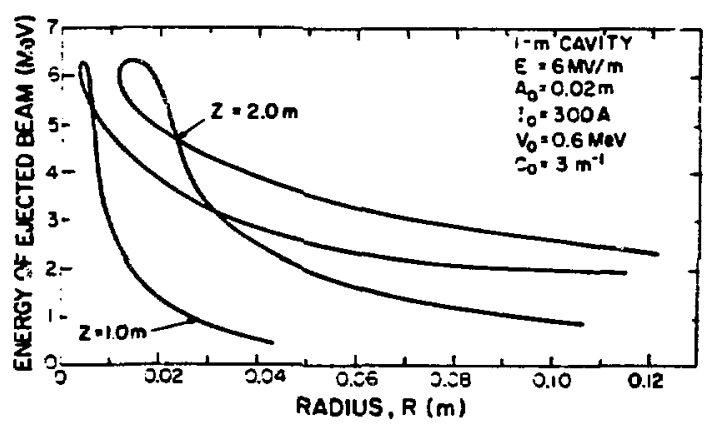

Fig. B-24.

Energy of ejected beam vs radius. 


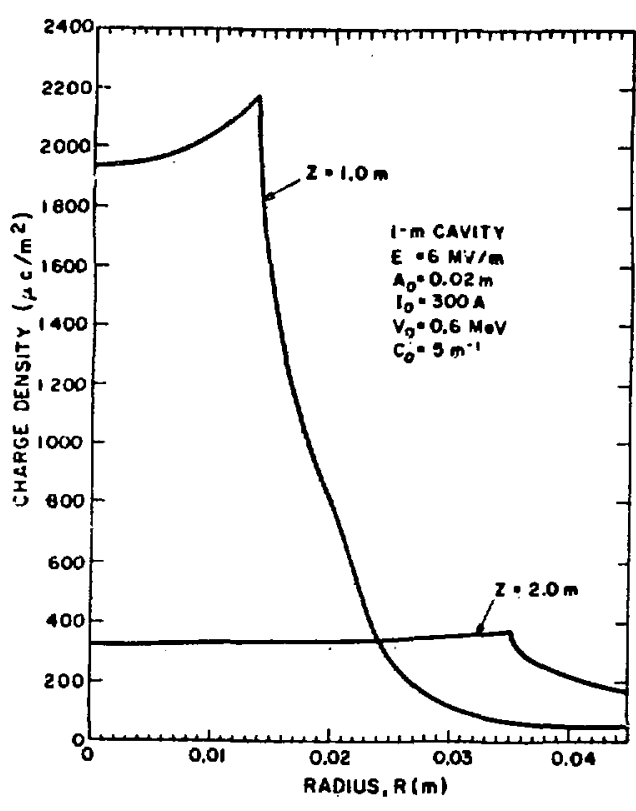

Fig. B-25.

Charge densily vs radius.

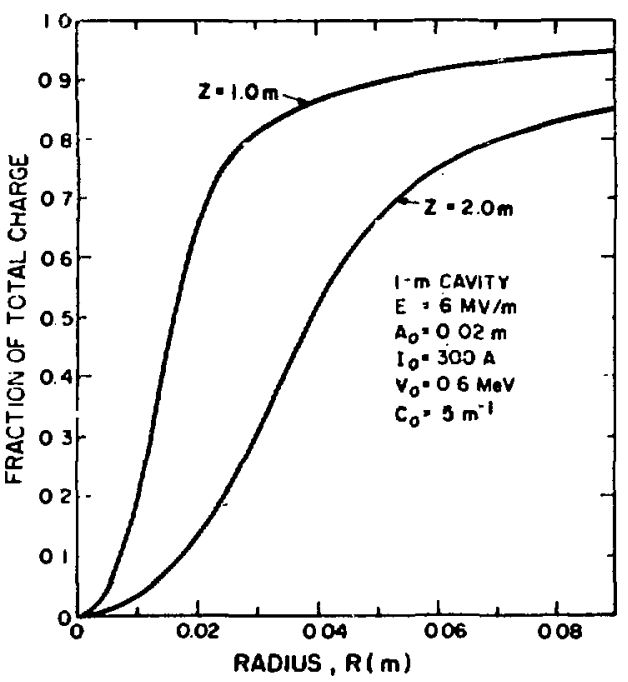

Fig. B-26.

Fraction of total charge hitting downstream wall within radius $R$.

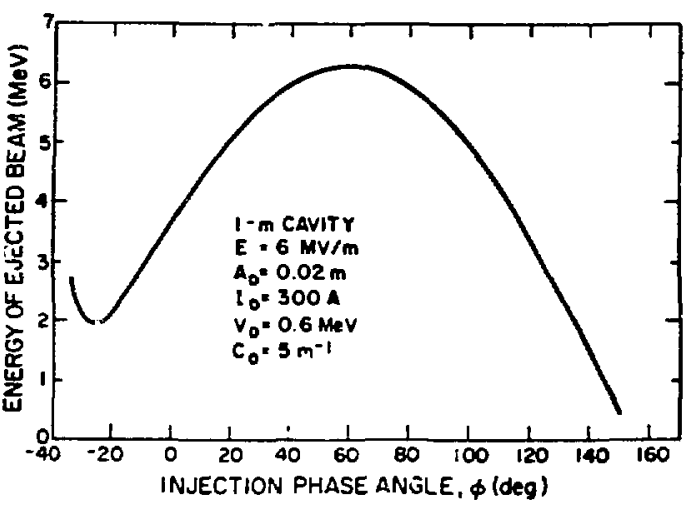

Fig. B-27.

Energy of ejected beam vs injection phase angle.

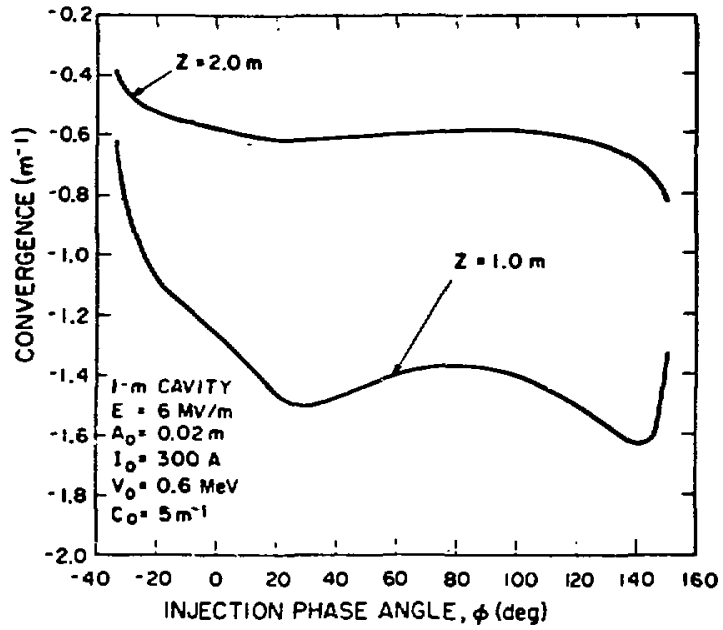

Fig. B-28.

Convergence of ejected beam vs injection phase angle.

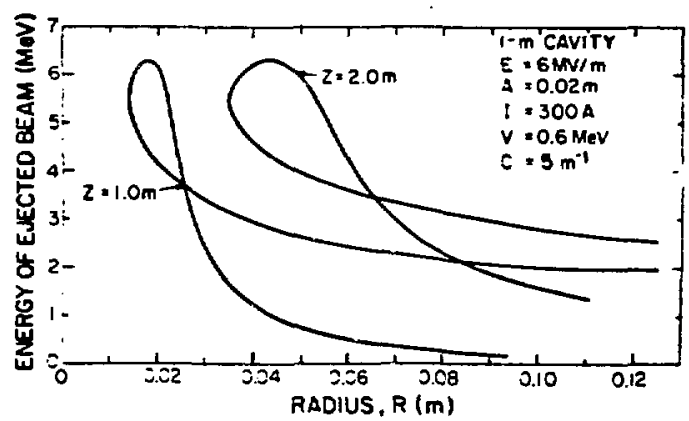

Fig. B-29.

Energy of ejected beam vs radius. 


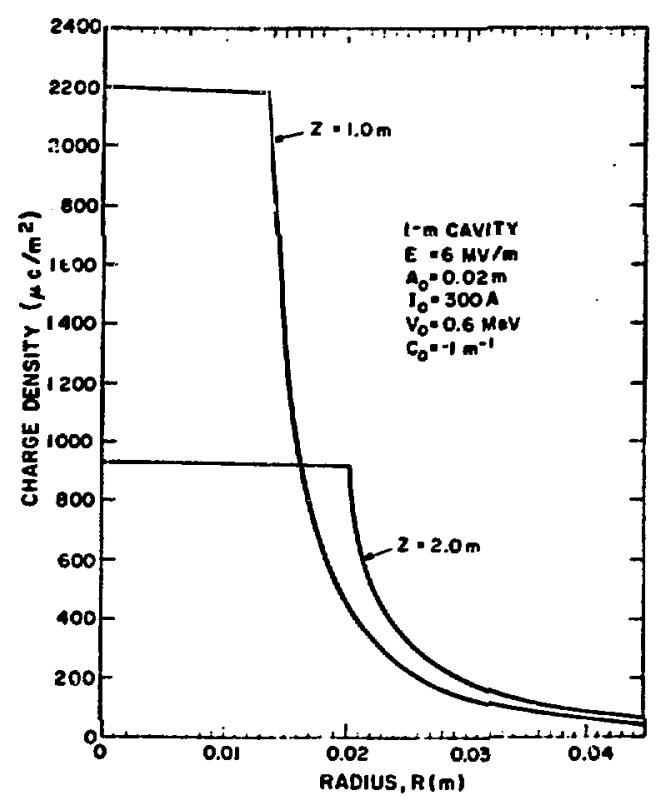

Fig. B-30.

Charge density vs radius.

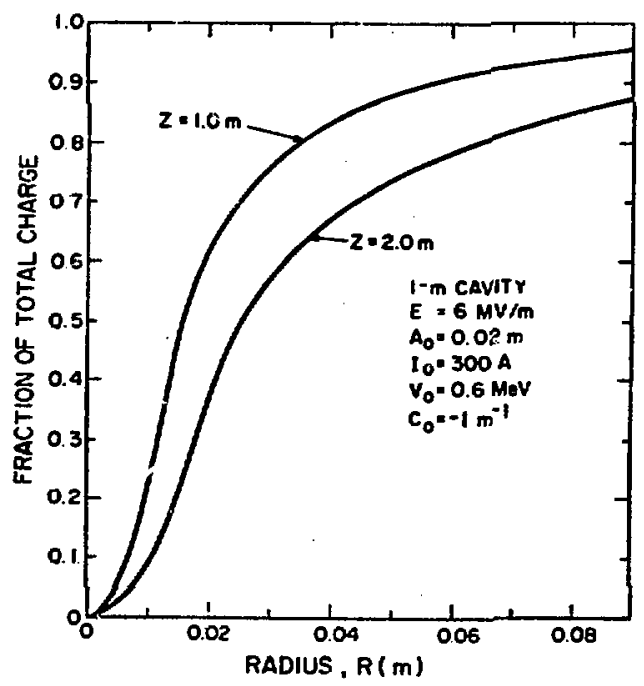

Fig. B-31.

Fraction of total charge hitting downstream wall within radius $R$.

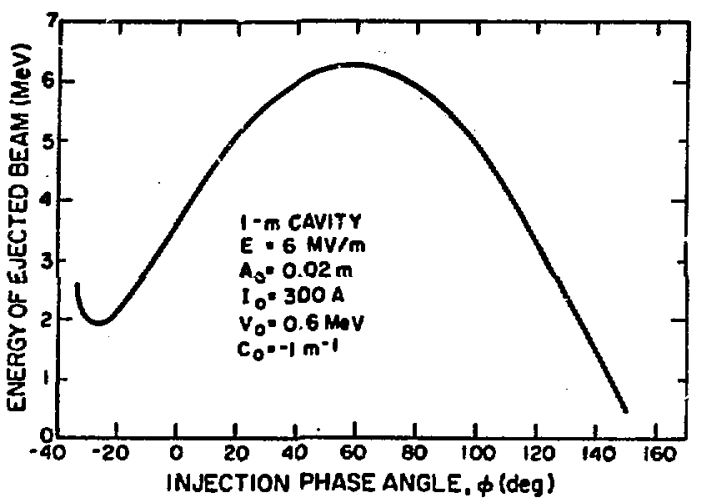

Fig. B-32.

Energy of ejected beam vs injection phase angle.

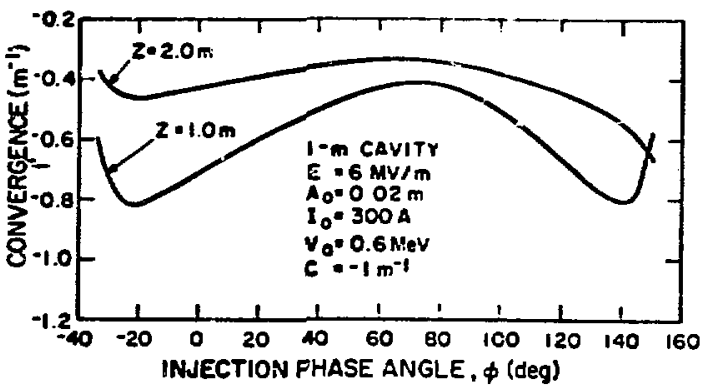

Fig. B-33.

Convergence of ejected beam vs injection phase angle.

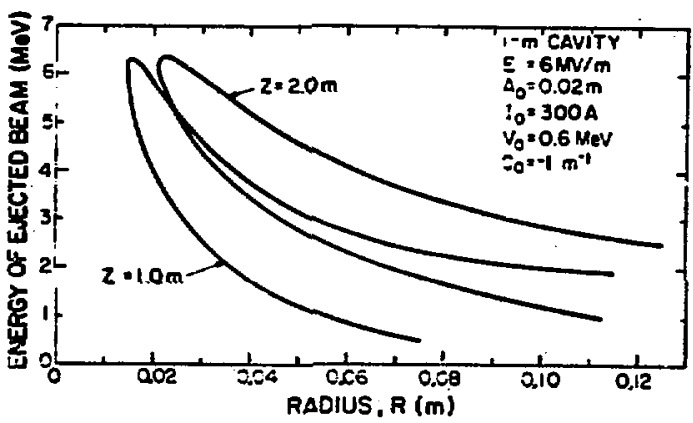

Fig. B-34.

Energy of ejected beam vs radius. 


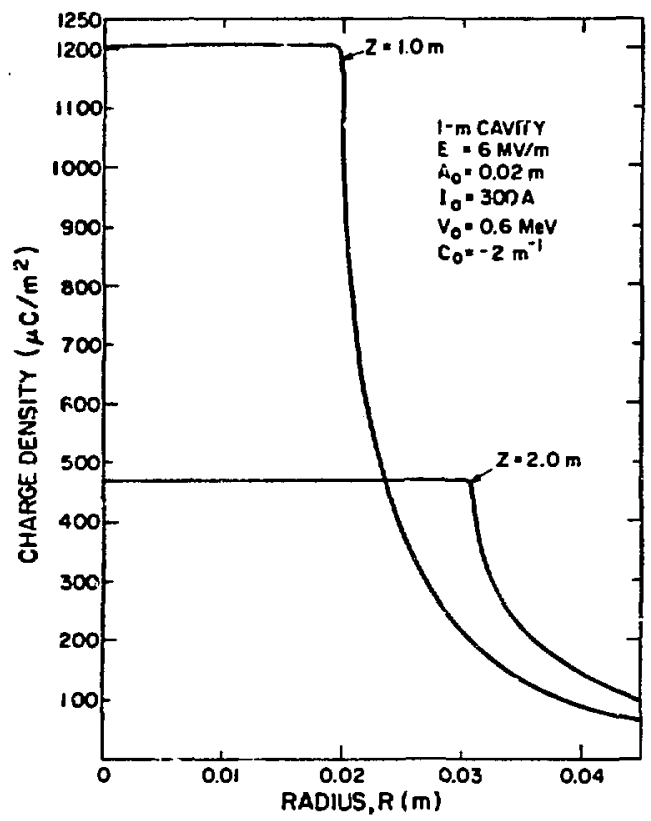

Fig. B-35.

Charge density vs radius.

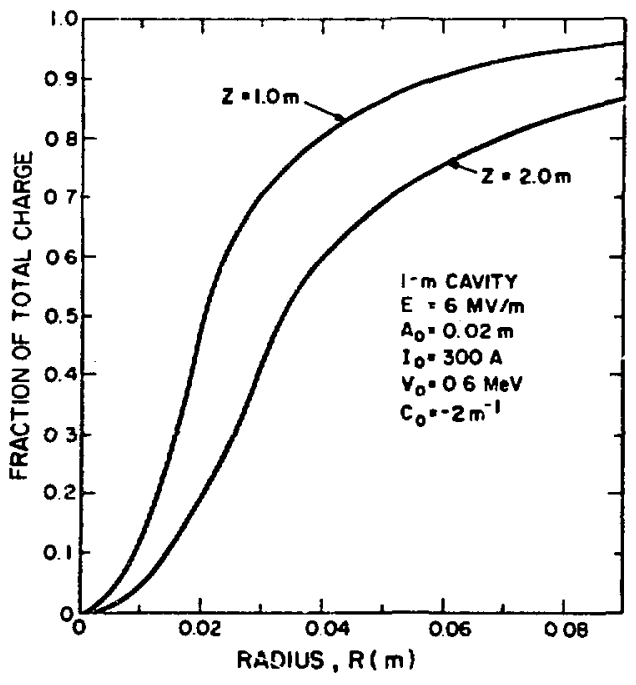

Fig. B-36.

Fraction of total charge hitting downstream wall within radius $R$.

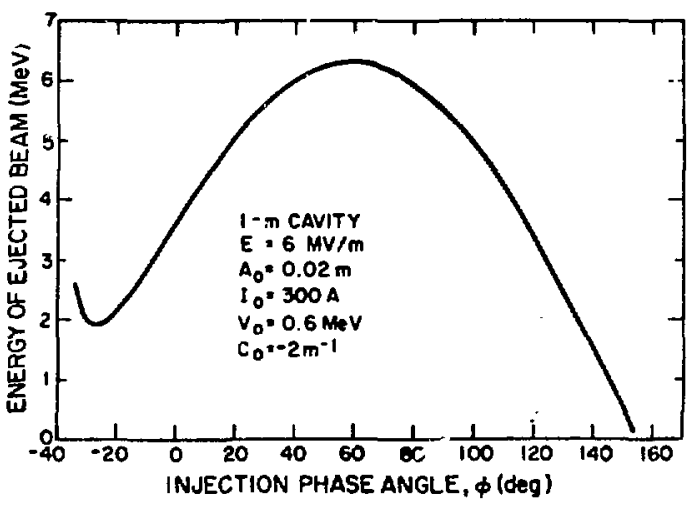

Fig. B-37.

Energy of ejected beam vs injection phase angle.

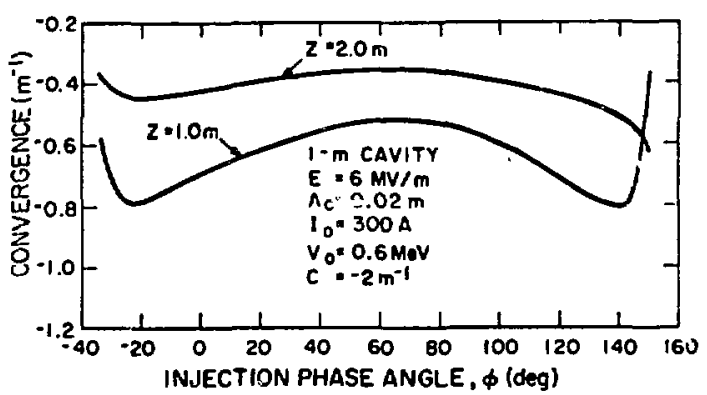

Fig. B-38.

Convergence of ejected beam vs injection phase angle.

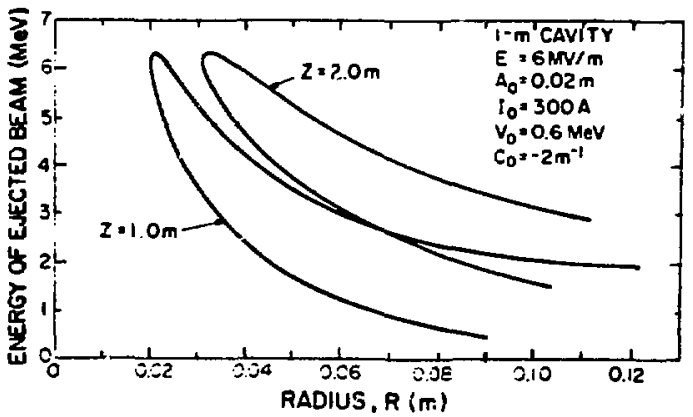

Fig. B.39.

Energy of ejected beam vs radius. 


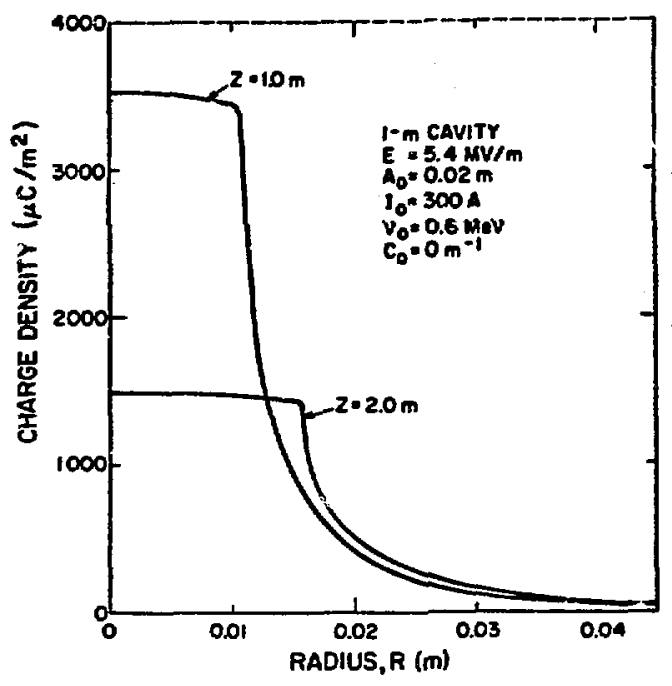

Fig. B-40.

Charge density vs radius.

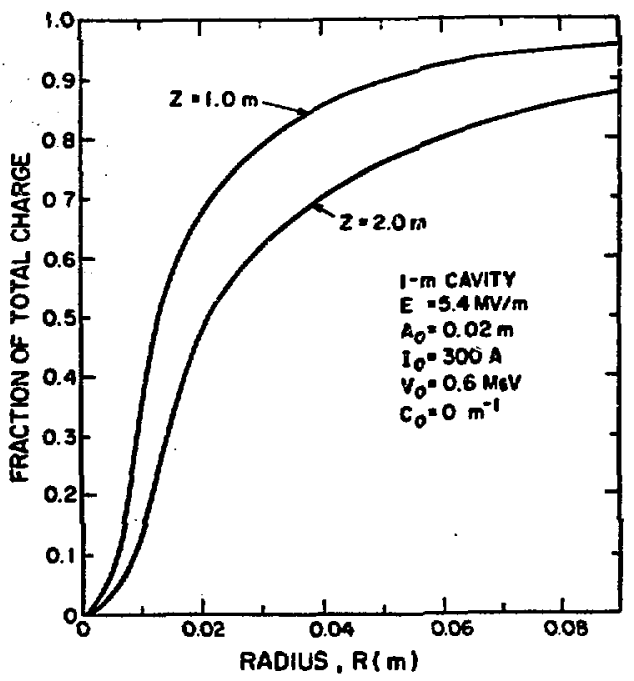

Fig. B-41.

Fraction of total charge hitting downstream wall within radius $\boldsymbol{R}$.

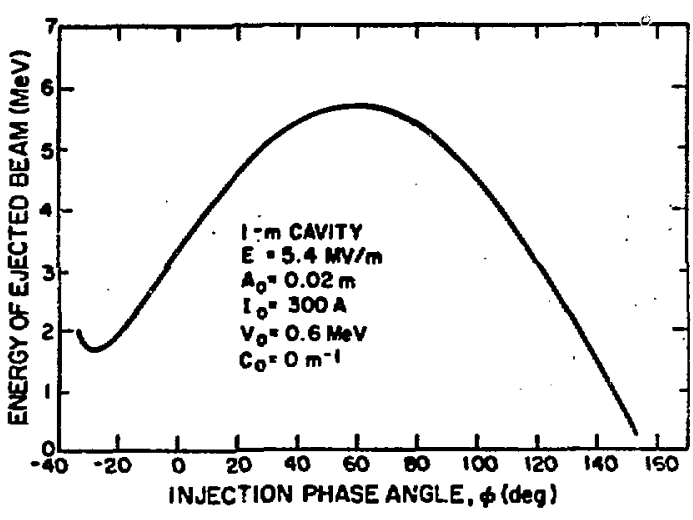

Fig. $8-42$.

Energy of ejected beam vs injection phase angle.

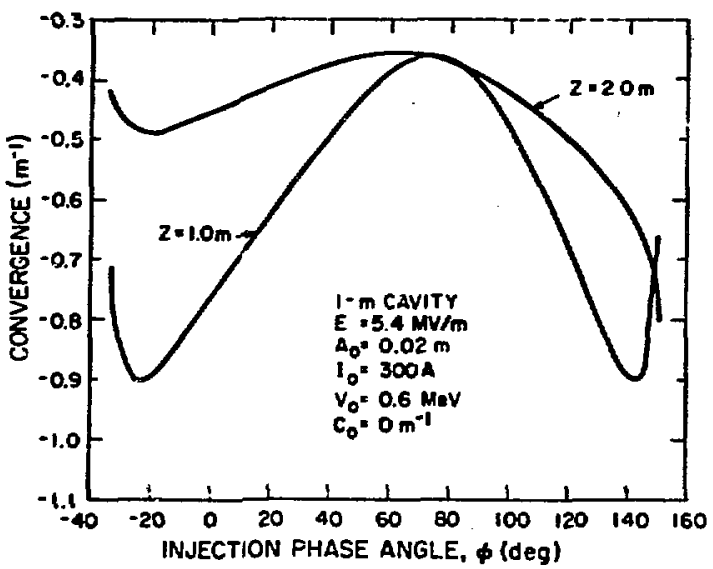

Fig. B-43.

Convergence of ejected beam vs injection phase angle.

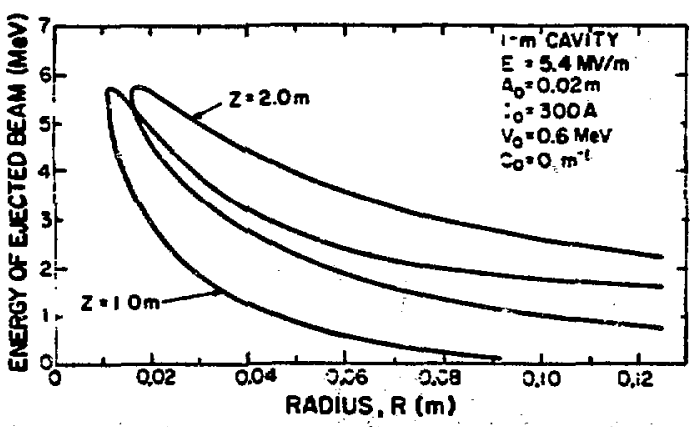

Fig. B-44.

Energy of ejected beam vs radius. 


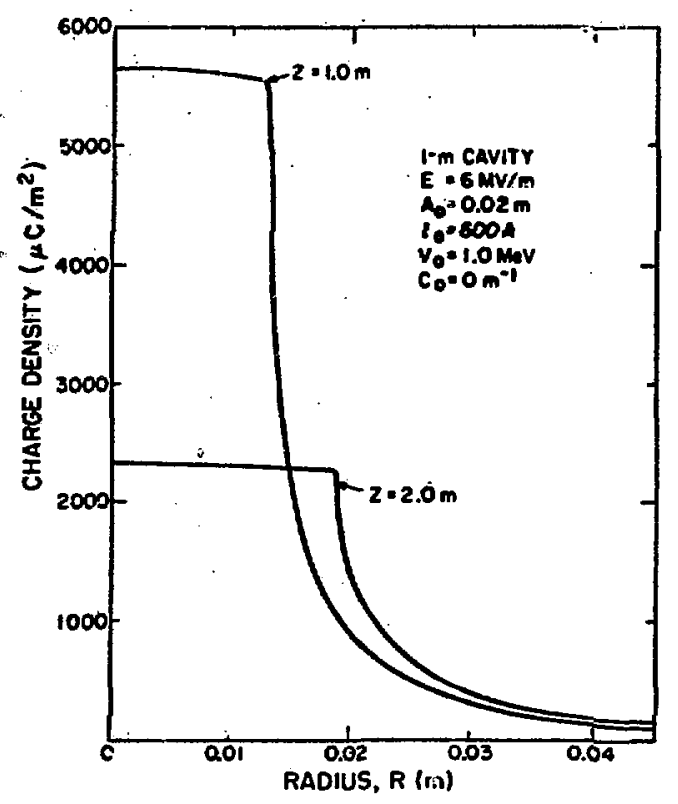

Fig. B-45.

Charge densify vs radius.

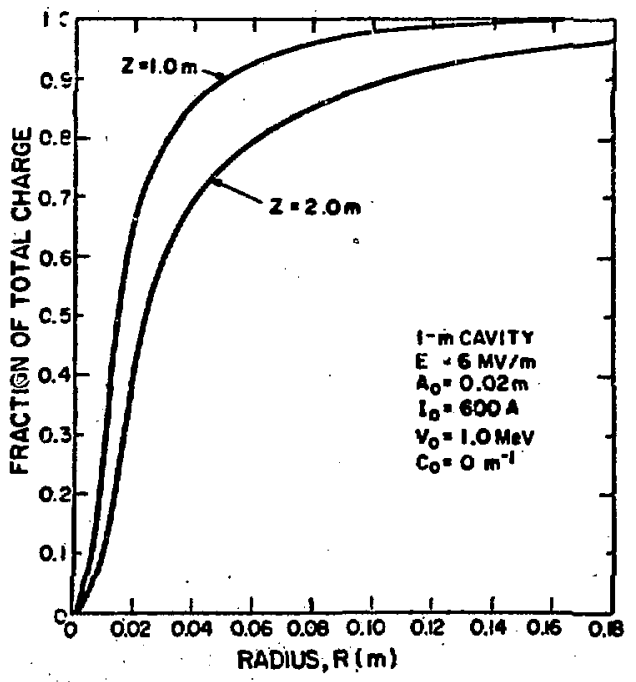

Fig. B-46.

Fraction of total charge hitting downstream wall within radius $\boldsymbol{A}_{\text {. }}$

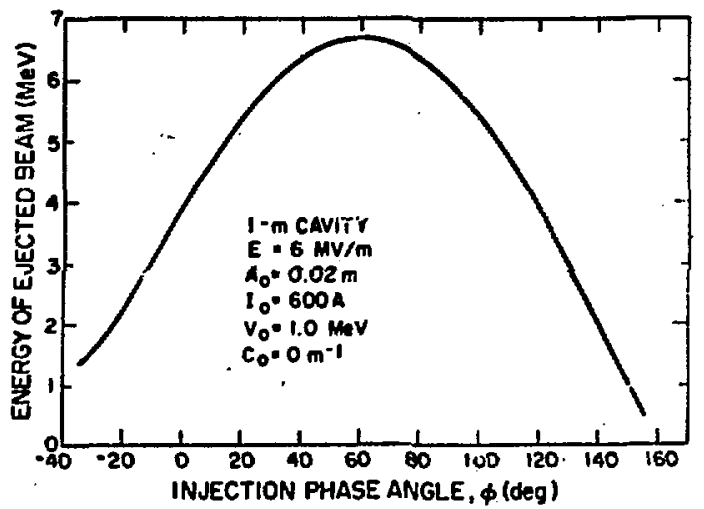

Fig. B-47.

Energy of ejected beam vs injection phase angle.

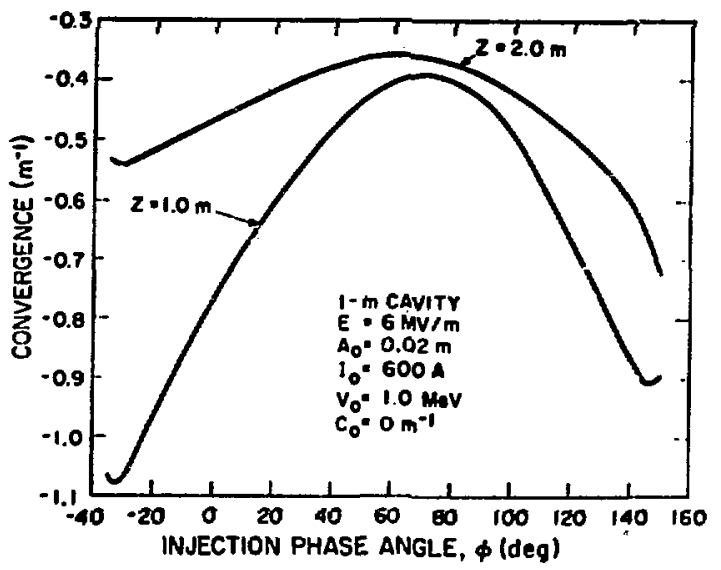

Fig. B-48.

Convergence of ejected beam vs injection phase angle.

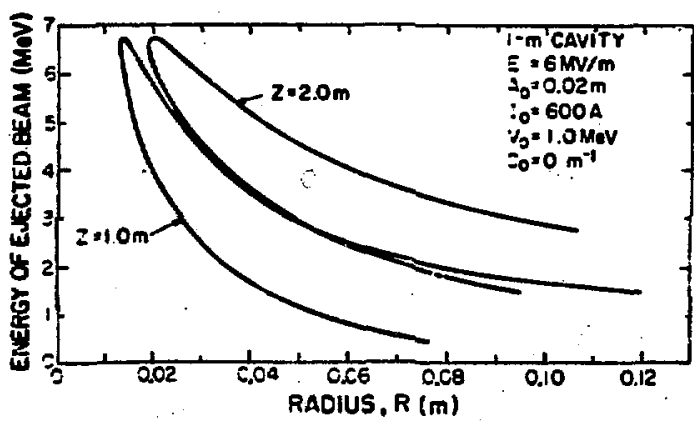

Fig. B-49.

Energy of ejected beam us radius. 


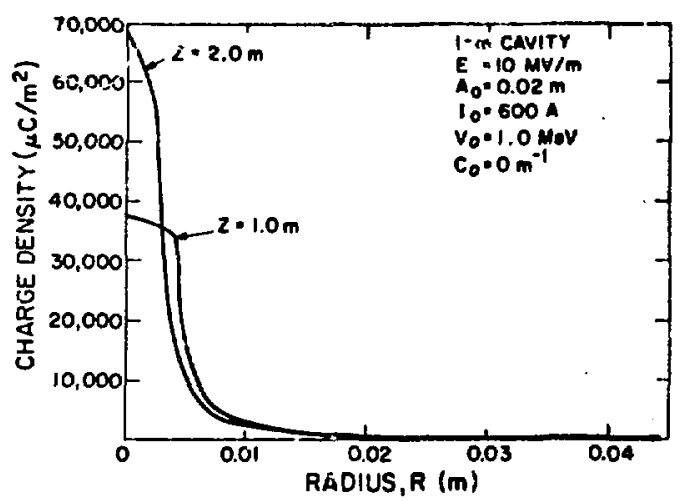

Fig. B-50.

Charge density vs radius:

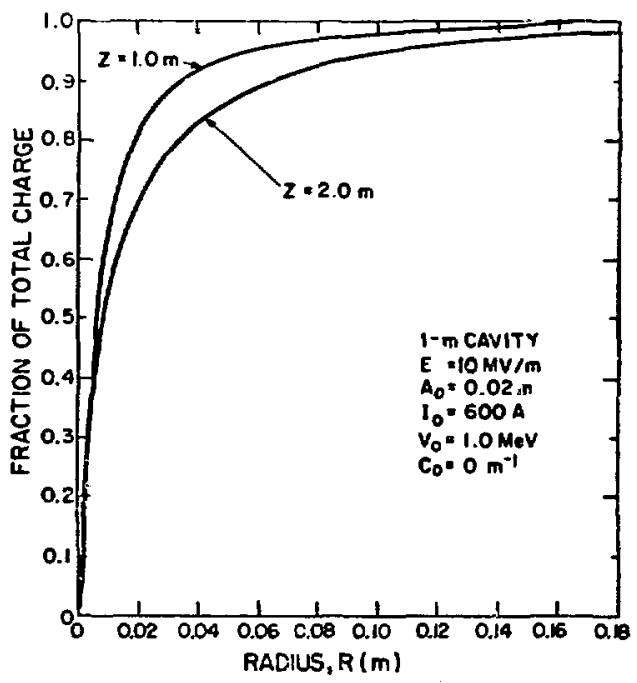

Fig. B-51.

Fraction of total charge hitting downstream wall within radius $R$.

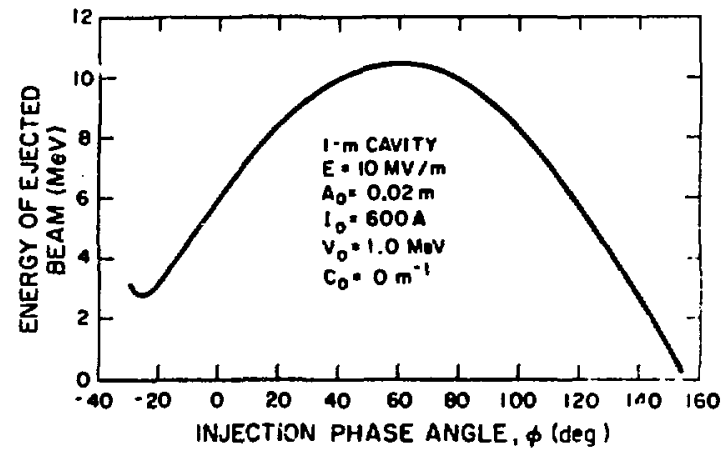

Fig. B-52.

Energy of ejected beam vs injection phase angle.

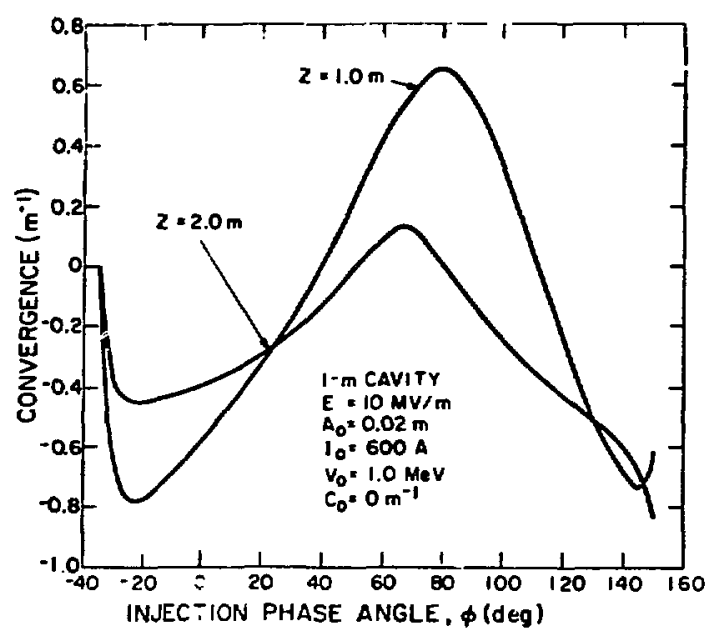

Fig. B-53.

Convergence of ejected beam vs injection phase angle.

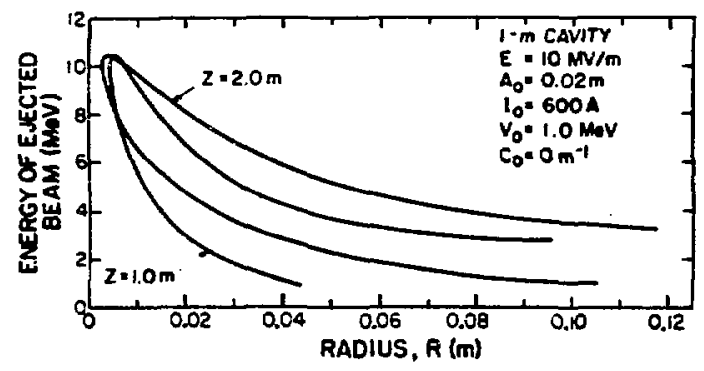

Fig. B-54.

Energy of ejected beam vs radius. 


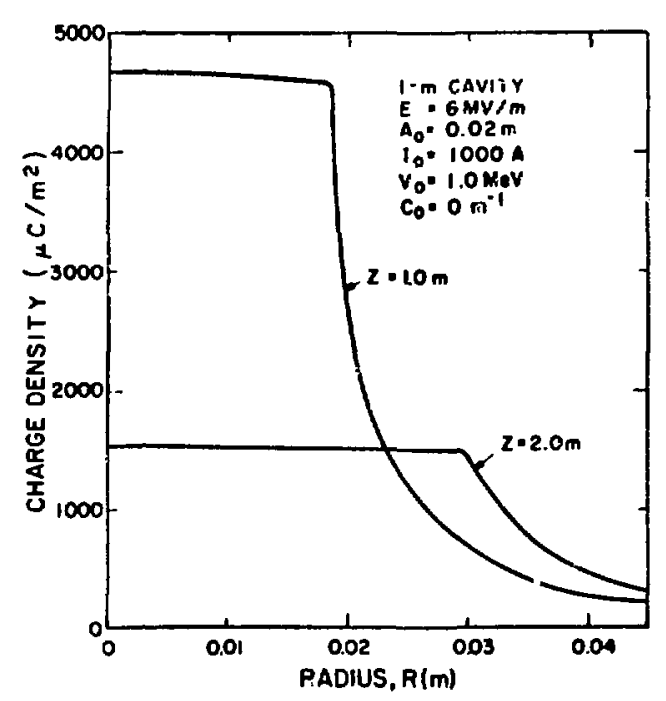

Fig. B-55.

Charge density vs radius.

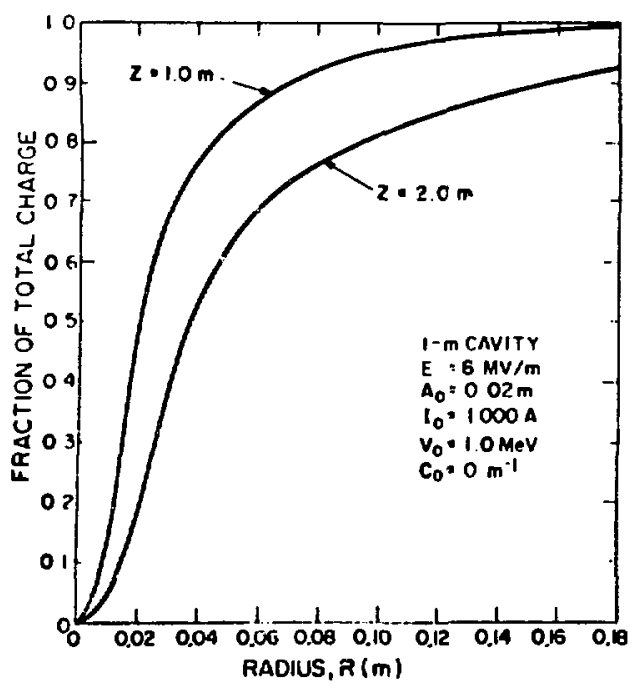

Fig. B-56.

Fraction of total charge hitting downstream wall within radius $A$.

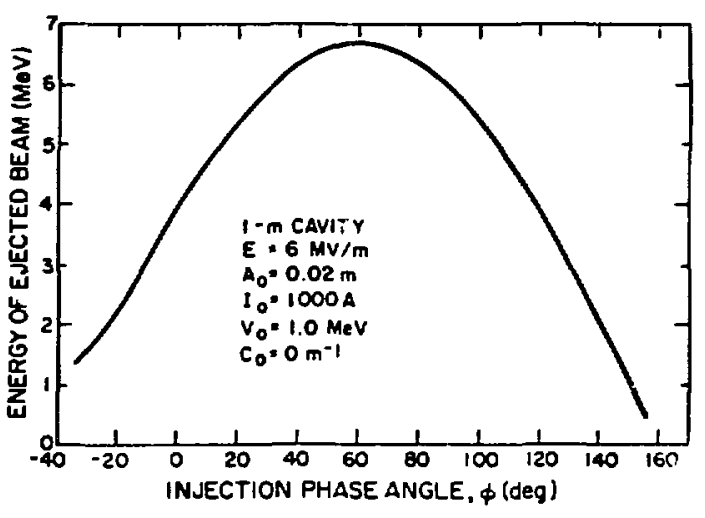

Fig. B-57.

Energy of ejected beam vs injection phase angle.

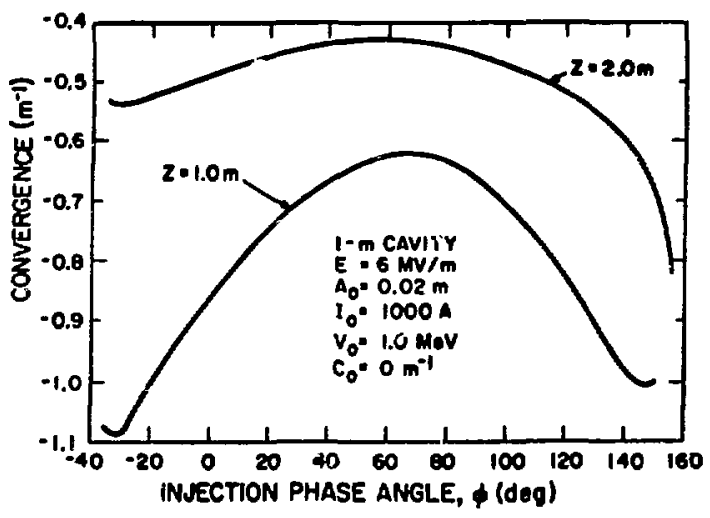

Fig. 8-58.

Convergence of ejected beam vs injection phase angle.

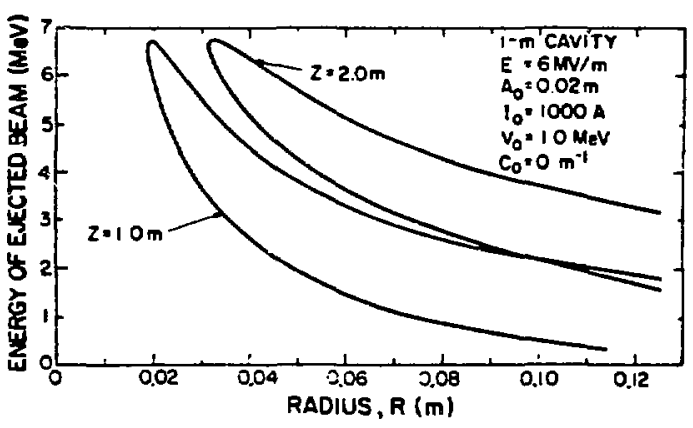

Fig. 8-59.

Energy of ejected beam vs radius. 


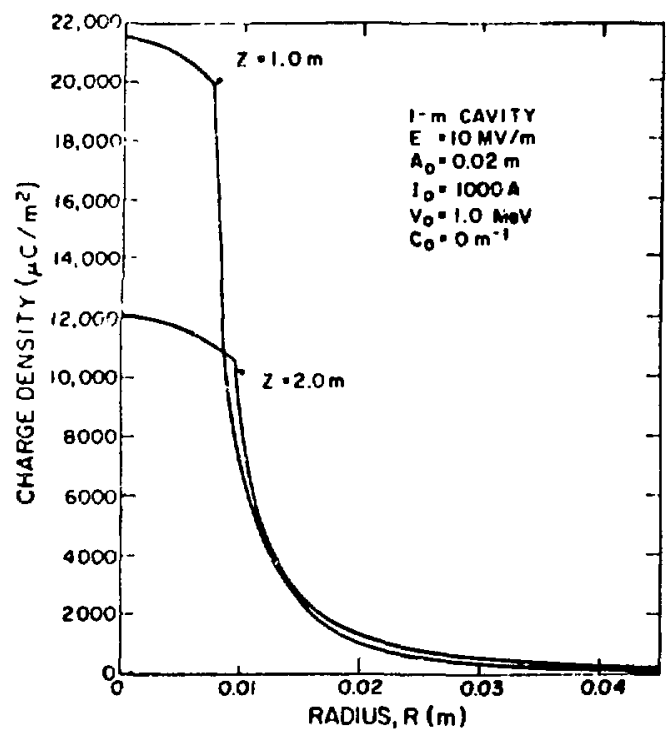

Fig. $B-60$

Charge density vs radius.

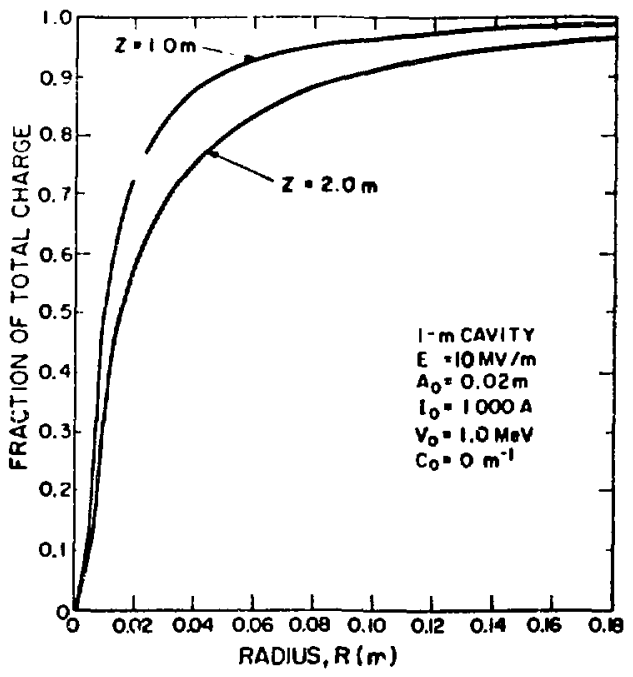

Fig. 8-61.

Fraction of total charge hitting downstream wall within radius $R$.

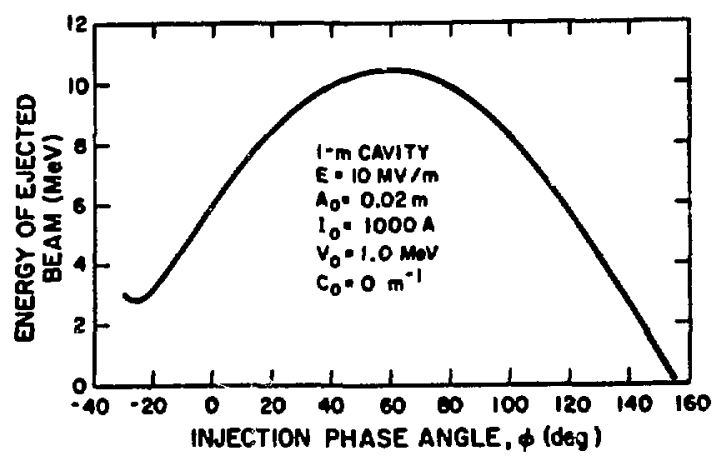

Fig. 8-62.

Energy of aiected team vs injection phase angle.

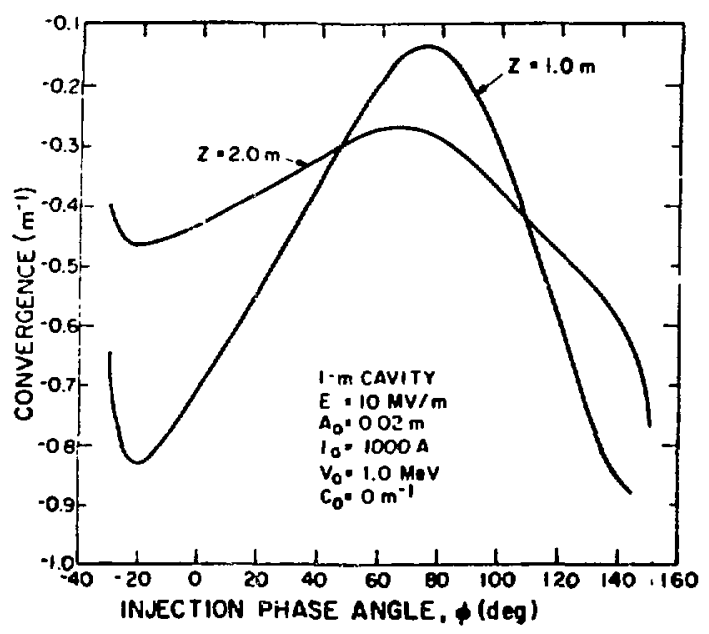

Fig. B-63.

Convergence of ejected bearr. vs injection phase angle.

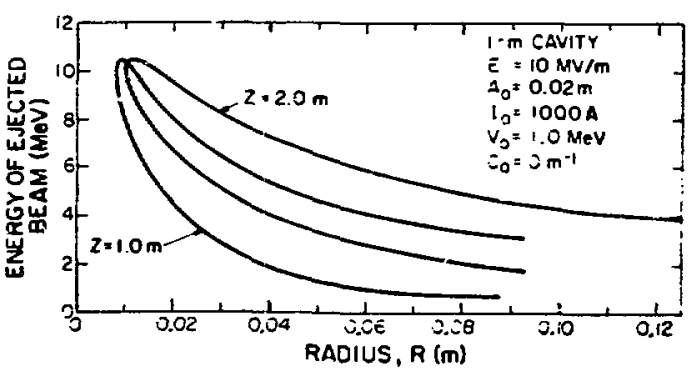

Fig. 8-64.

Energy of ejected beam vs radius. 


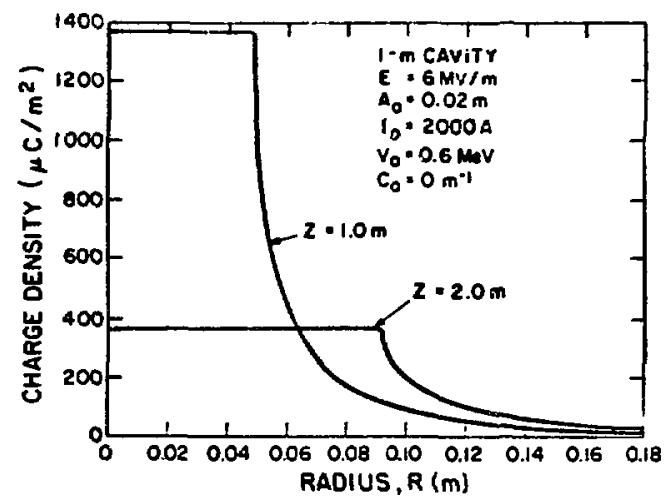

Fig. B-65.

Charge density vs radius.

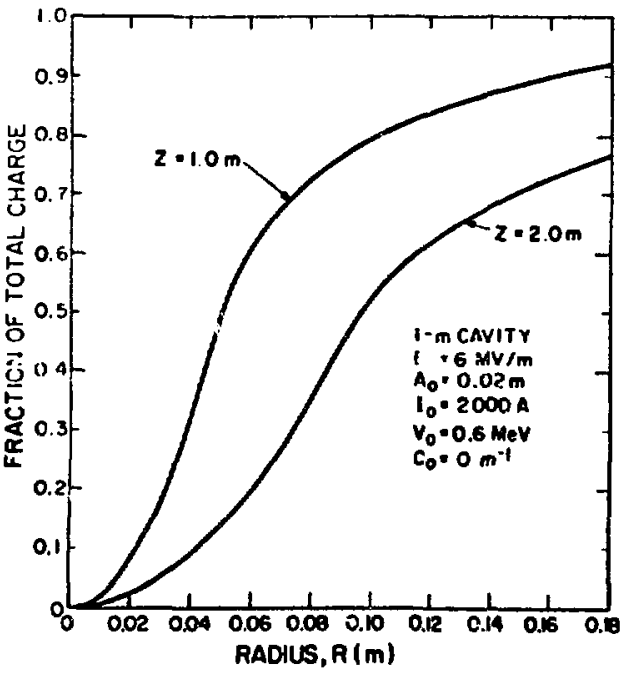

Fig. B-66.

Fraction of total charge hitting downstream wall within radius $R$.

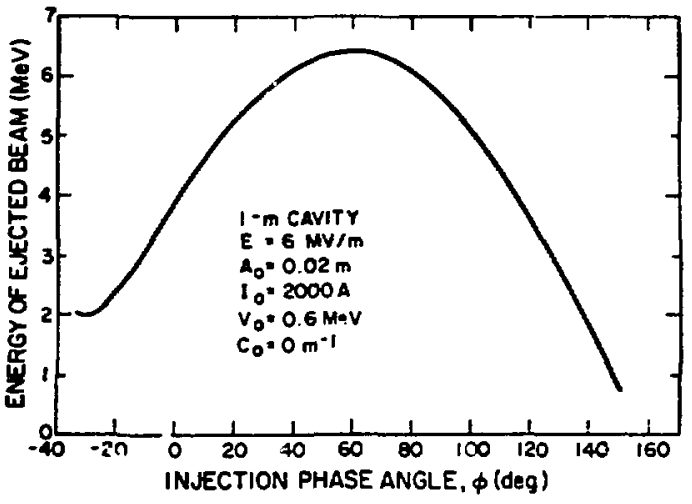

Fig. B-67.

Energy of ejected beam vs injection phase angle.

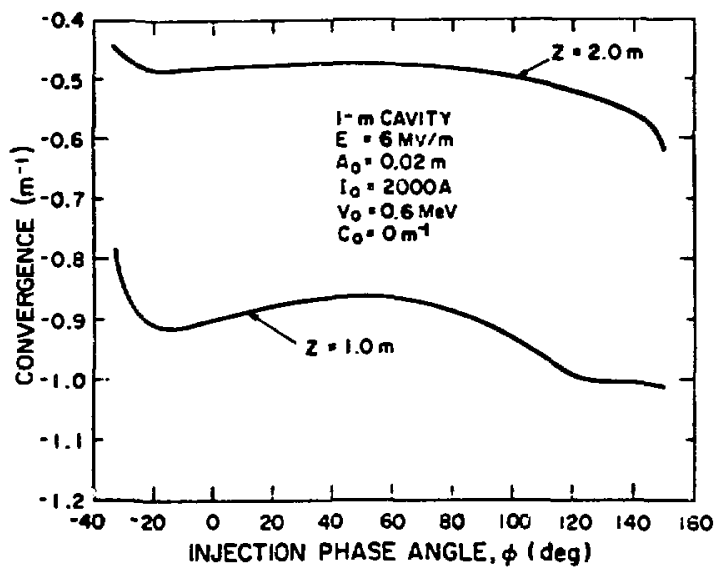

Fig. B-68.

Convergence of ejected beam vs injection phase angle.

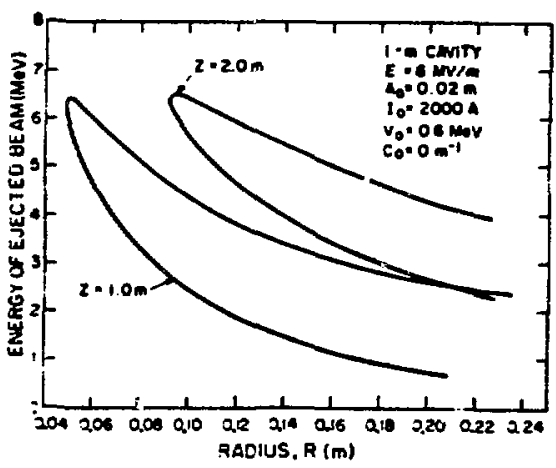

Fig. B-69.

Energy of ejected beam vs radius. 


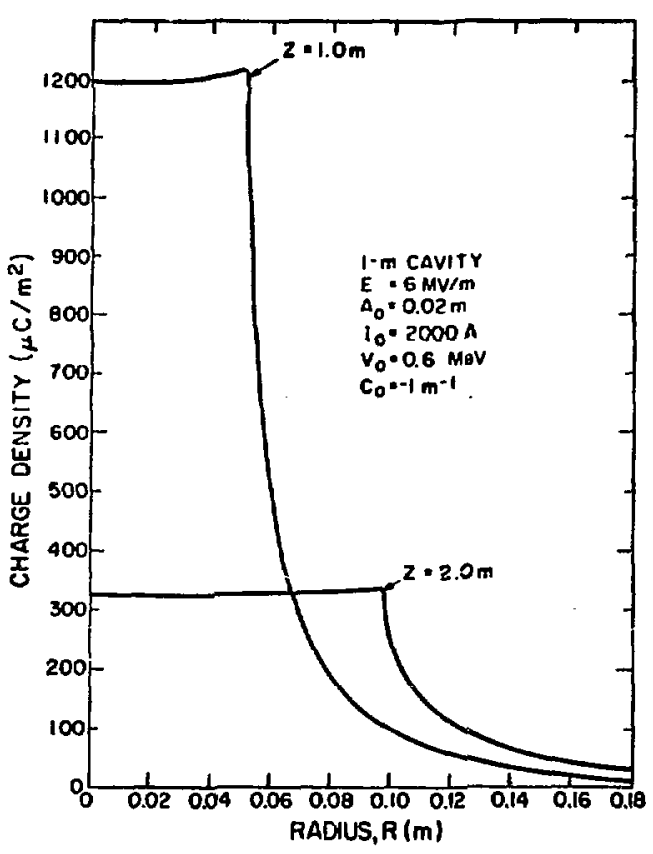

Fig. B-70.

Charge density vs radius.

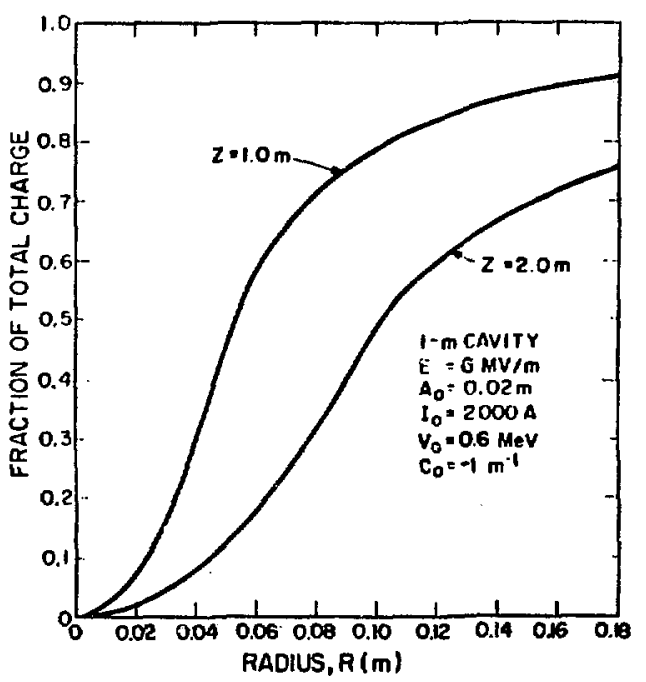

Fig. 8-71.

Fraction of total charge hitting downstream wall within radius $A$.

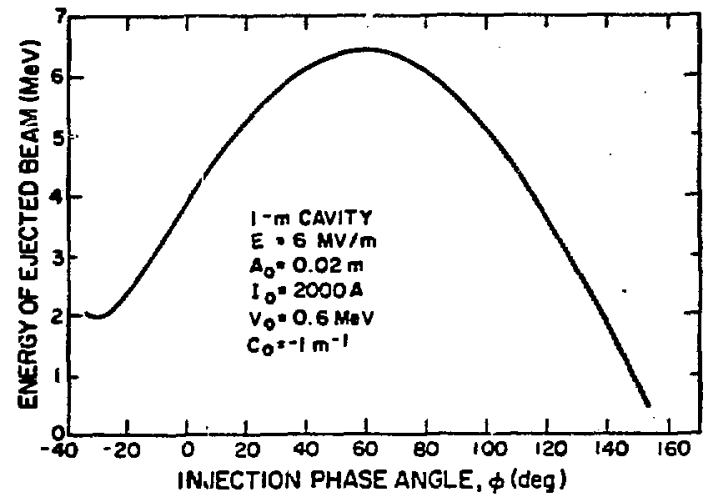

Fig. B-72.

Energy of ejected beam vs injection phase angle.

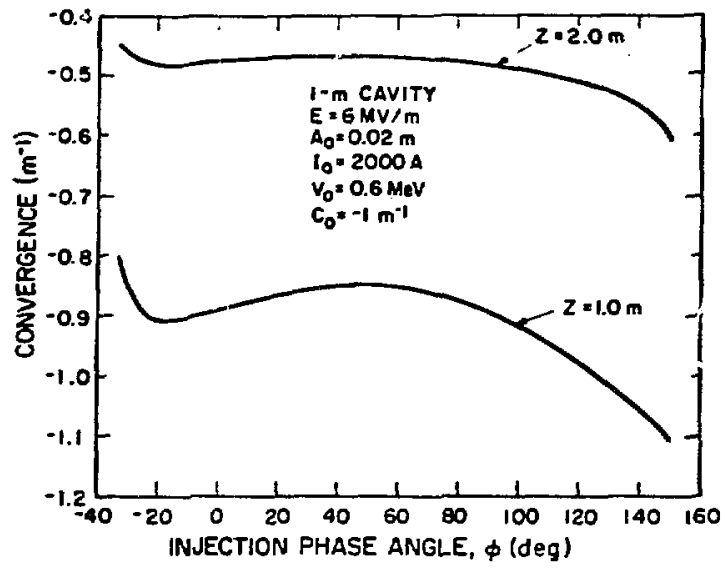

Fig. B-73.

Convergence of ejected beam vs injection phase angle.

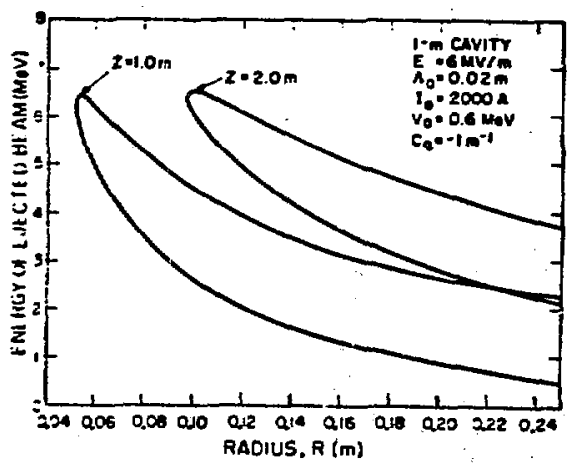

Fig. B-74.

Energy of ejected beam vs radius. 


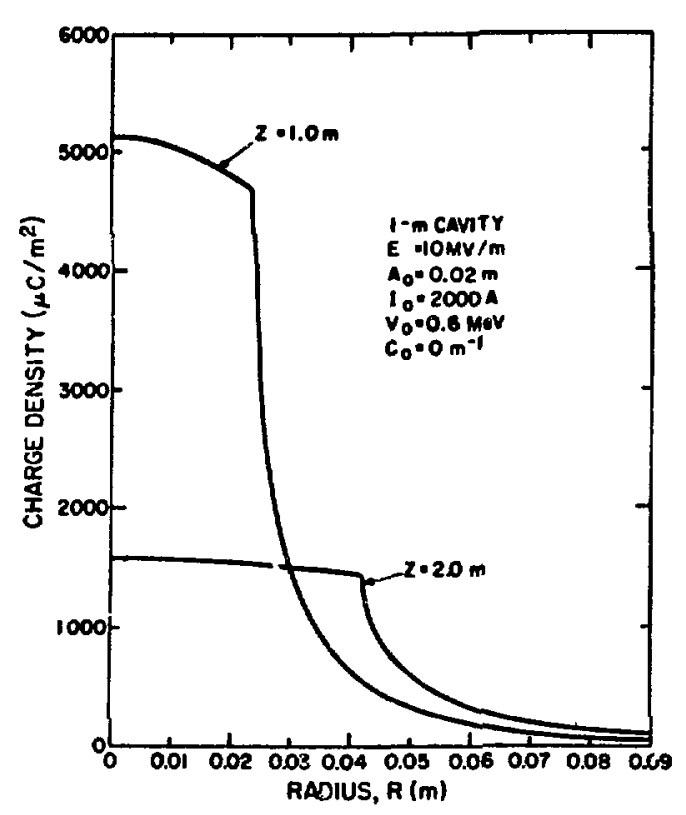

Fig. B-75.

Charge density vs radius.

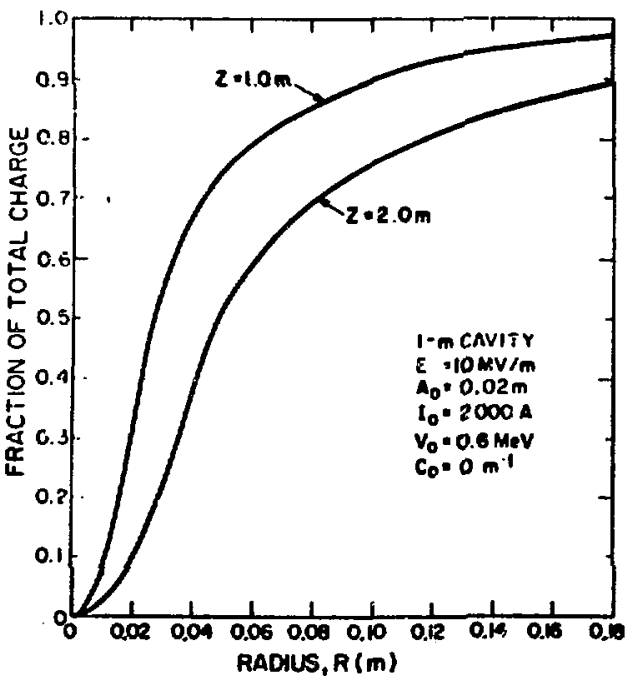

Fig. B-76.

Fraction of total charge hitting downstream wall within radius $R$.

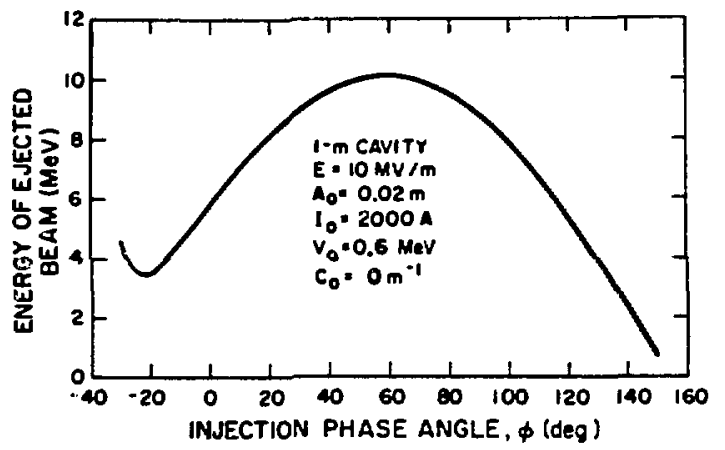

Fig. B-77.

Energy of ejected beam vs injection phase angla.

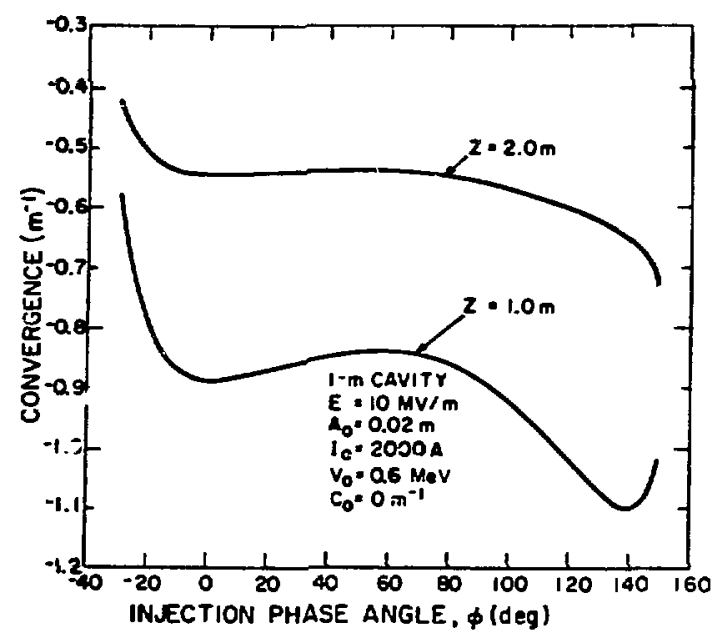

Fig. B-78.

Convergence of ejested beam vs injection phase angle.

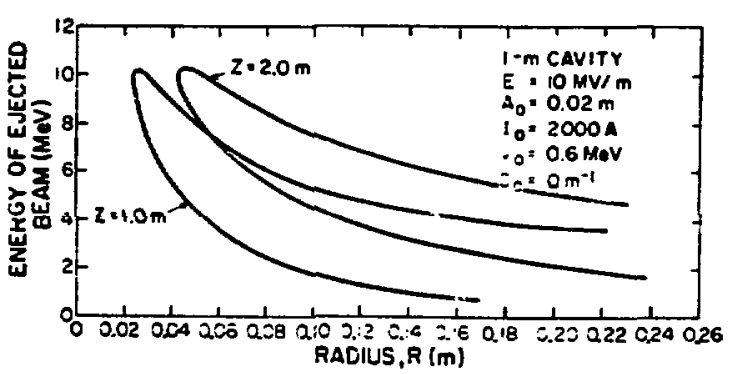

Fig. B-79.

Energy of ejected beam vs radius. 


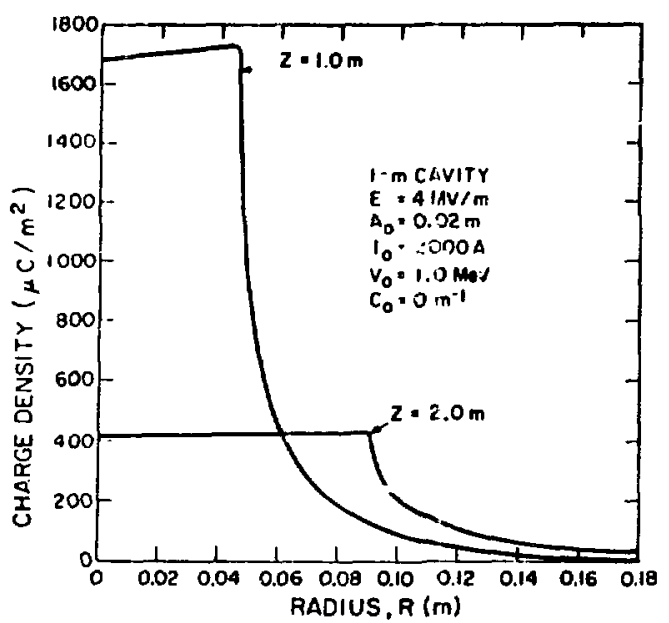

Fig. B-80.

Charge density vs radius.

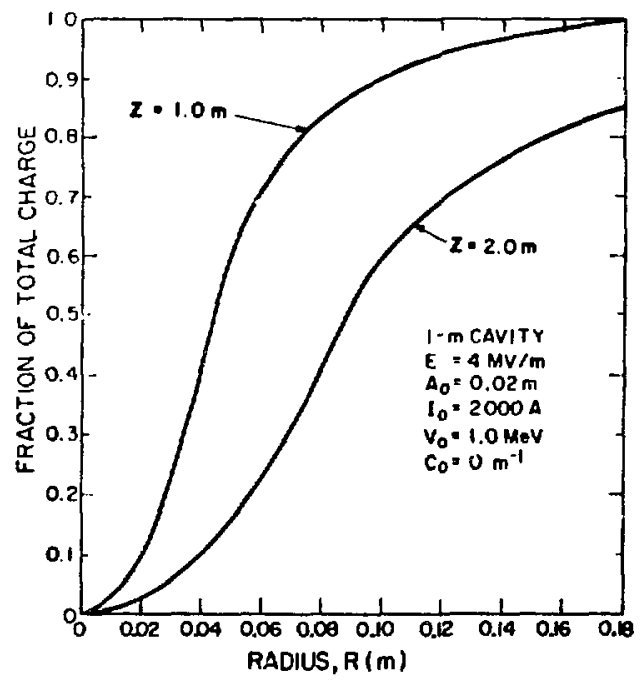

Fig. B-81.

Fraction of total charge hitting downstream wall within radius $R$.

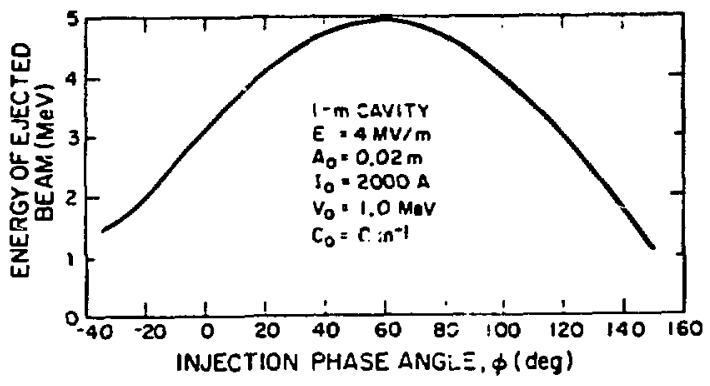

Fig. B-82.

Energy of ejected beam vs injection phase angle.

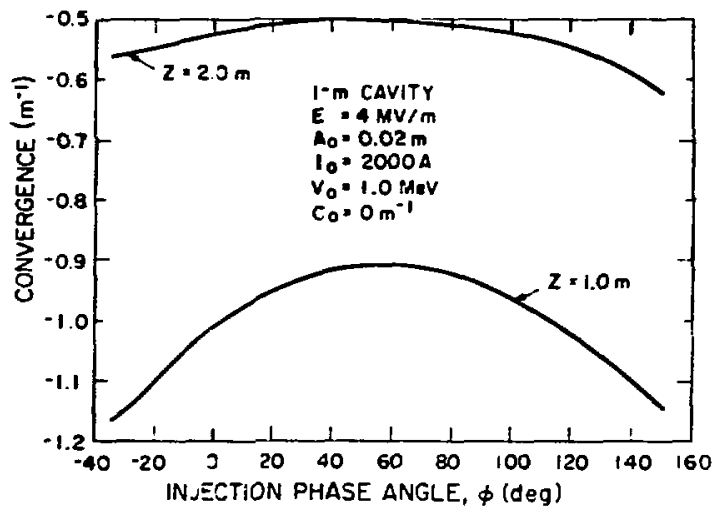

Fig. B-83.

Convergence of ejected beam vs injection phase angle.

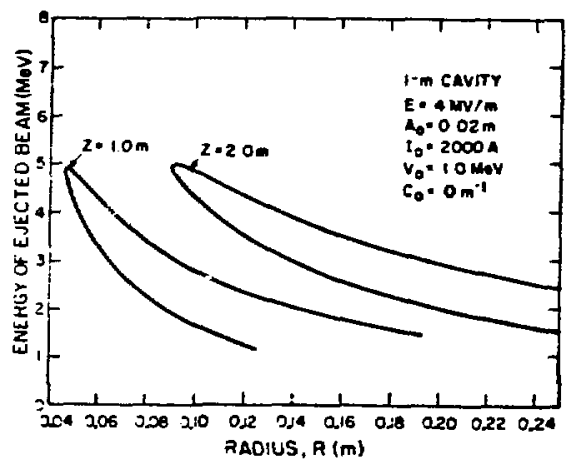

Fig. B-84.

Energy of ejected beam vs radius. 


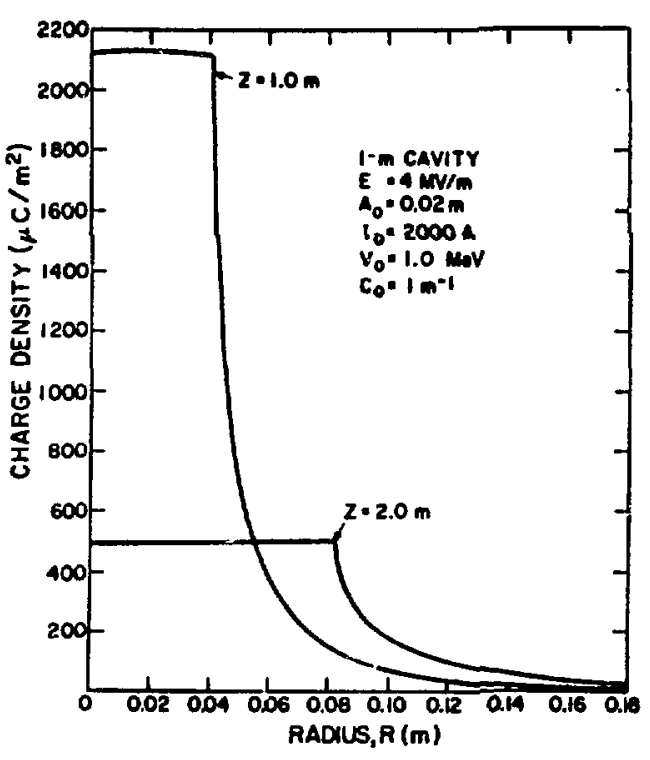

Fig. B-85.

Charge density vs radius.

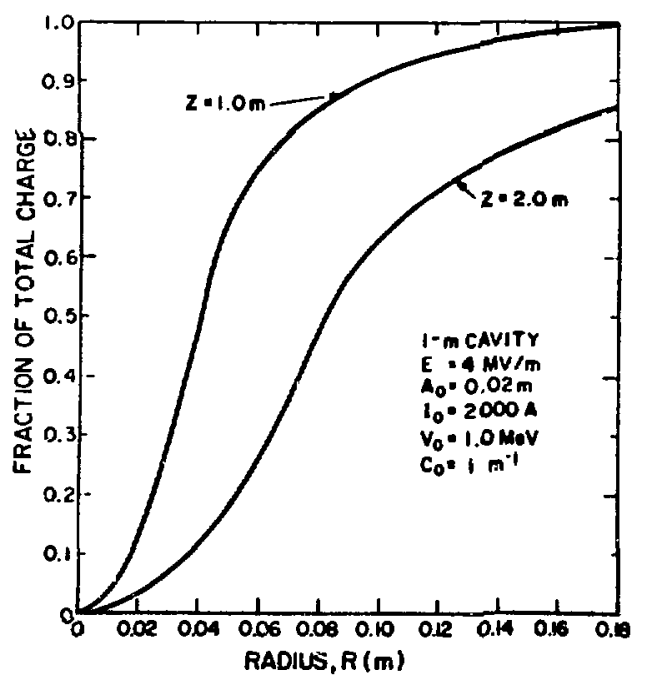

Fig. B-86.

Fraction of total charge hitting downstream wall within radlus $R$.

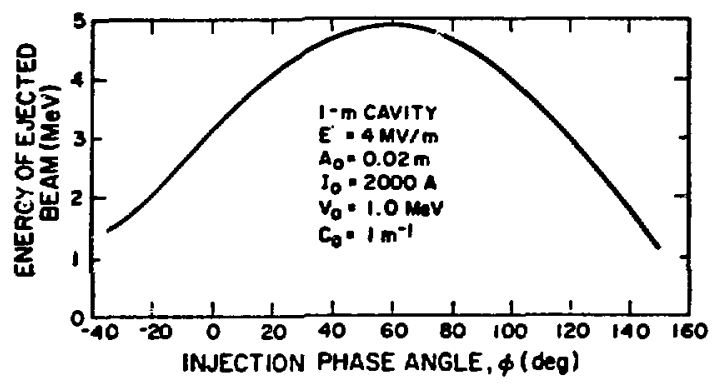

Fig. B-87.

Energy of ejected beam vs injection phase angle.

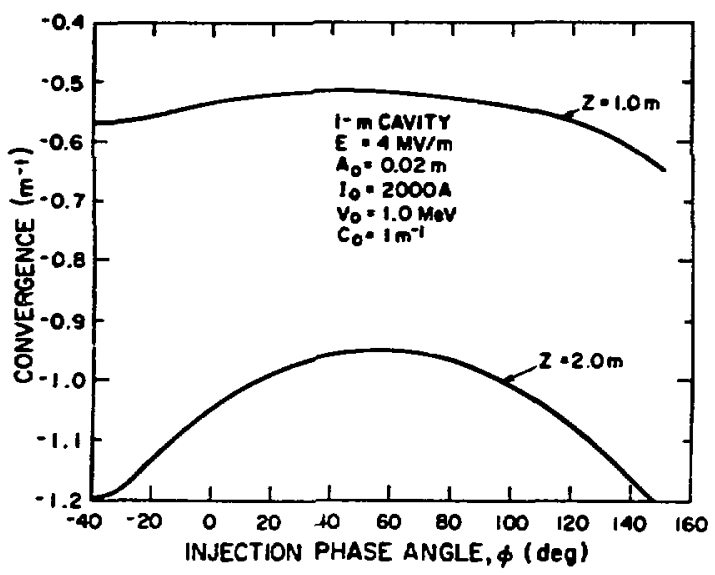

Fig. B-88.

Convergence of ejected beam vs injection phase angle.

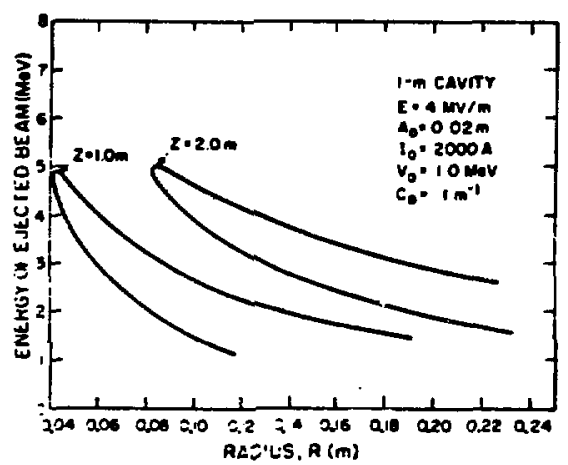

Fig. B-89.

Energy of ejected beam vs radius. 


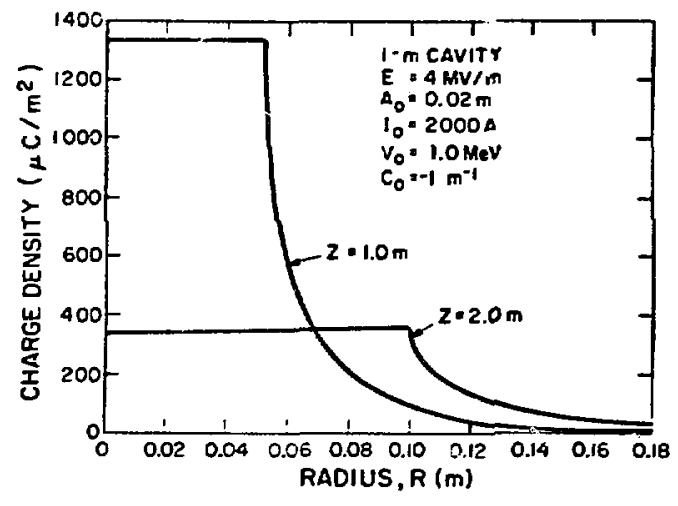

Fig. B-90.

Charge density vs radius.

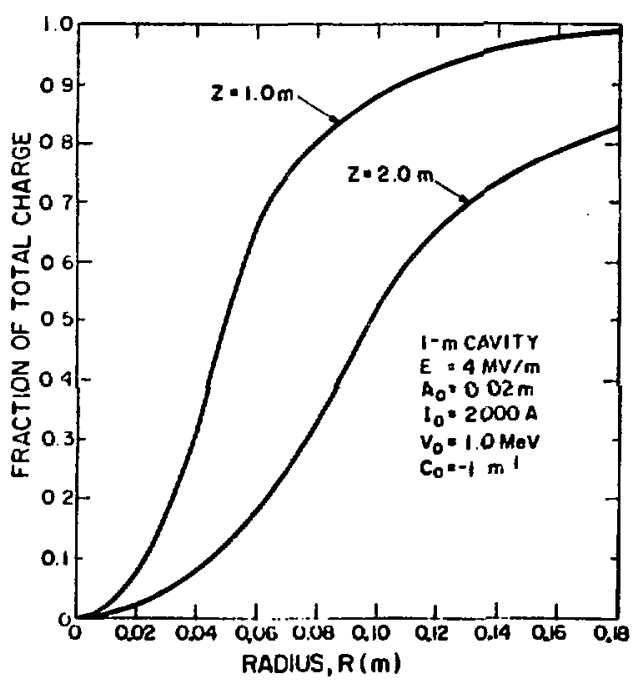

Fig. B-91.

Fraction of total charge hitting downstream wall within radius $R$.

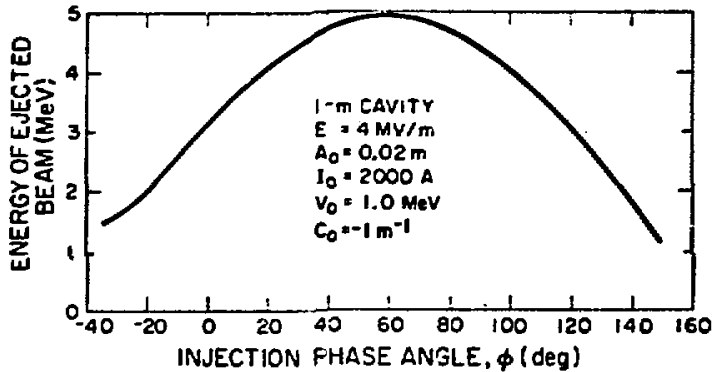

Fig. B-92.

Energy of ejected beam vs injection phase angle.

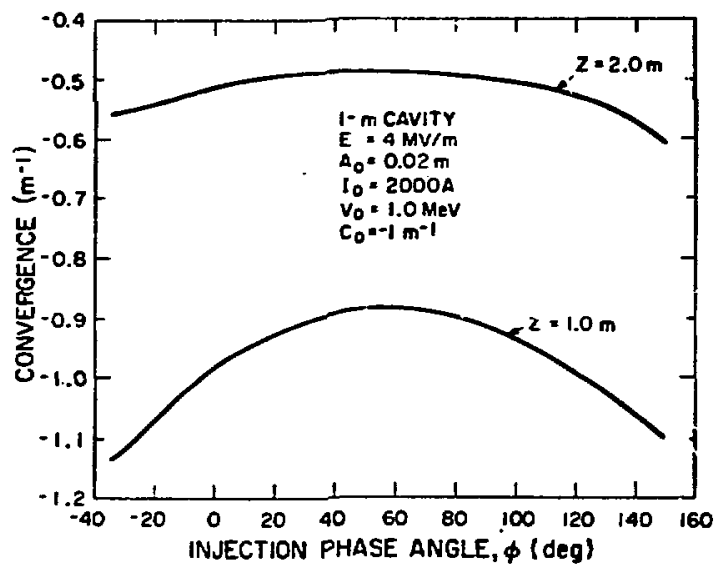

Fig. B-93.

Convergence of ejected beam vs injection phase angle.

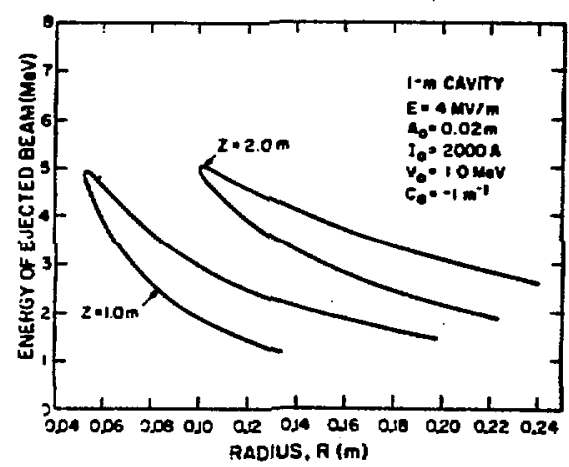

Fig. B-94.

Energy of ejected beam vs radius. 


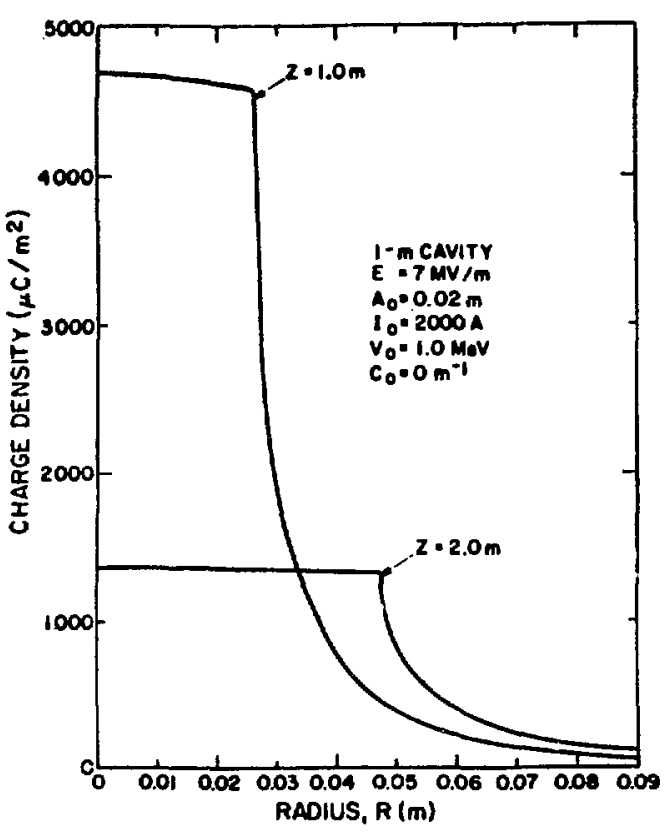

Fig. B-95.

Charge density vs radius.

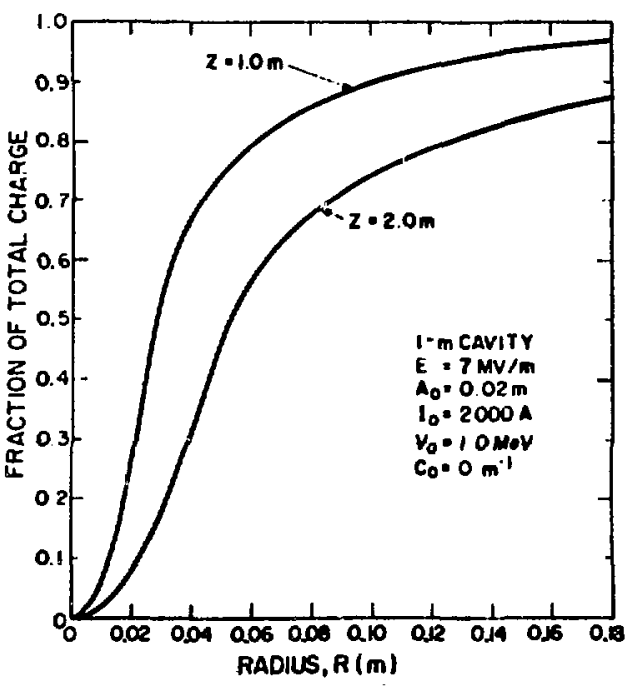

Fig. 8-96.

Fraction of total charge h/tting downstream wall within radius $R$.

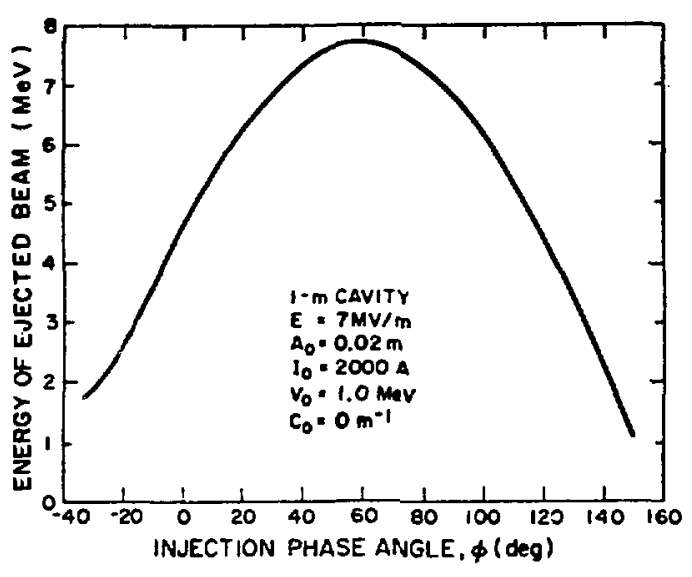

Fig. B-97.

Energy of ejected beam vs injection phase angle.

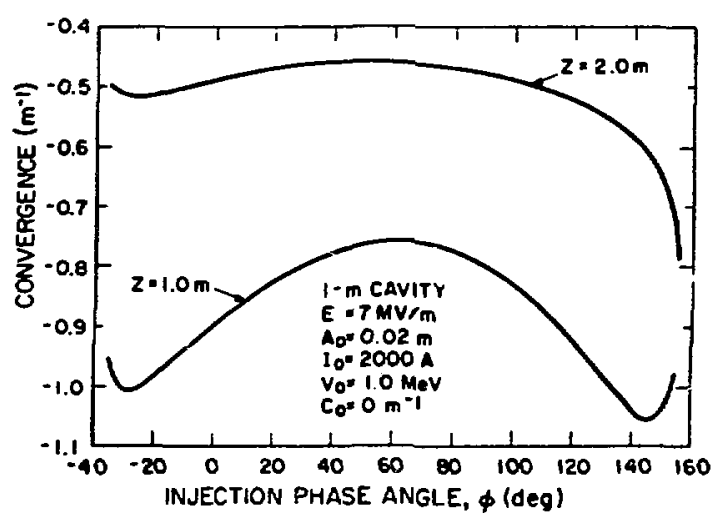

Fig. B-98.

Convergence of ejected beam vs injection phase angle.

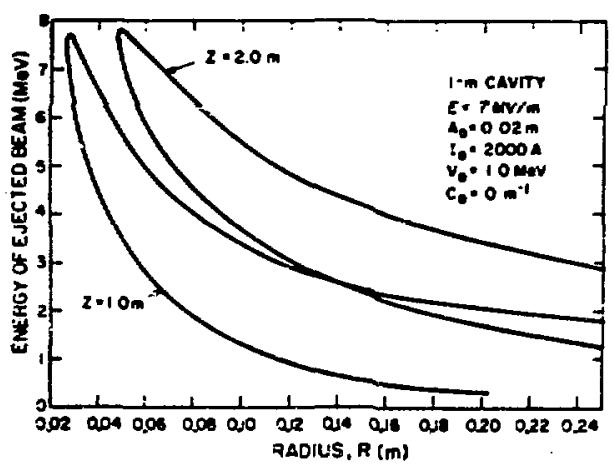

Fig. B-99.

Energy of ejected beam vs radius. 


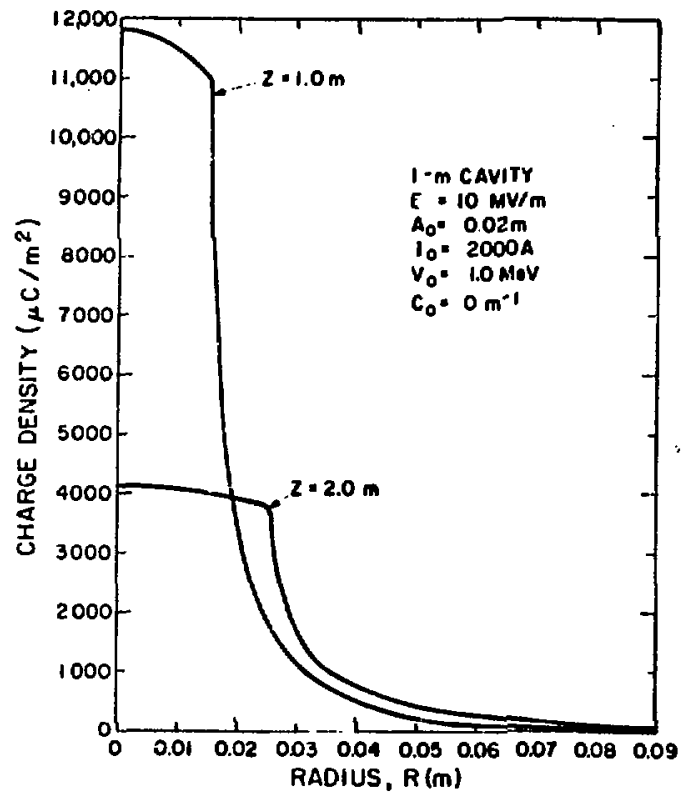

Fig. E-100.

Charge density vs radius.

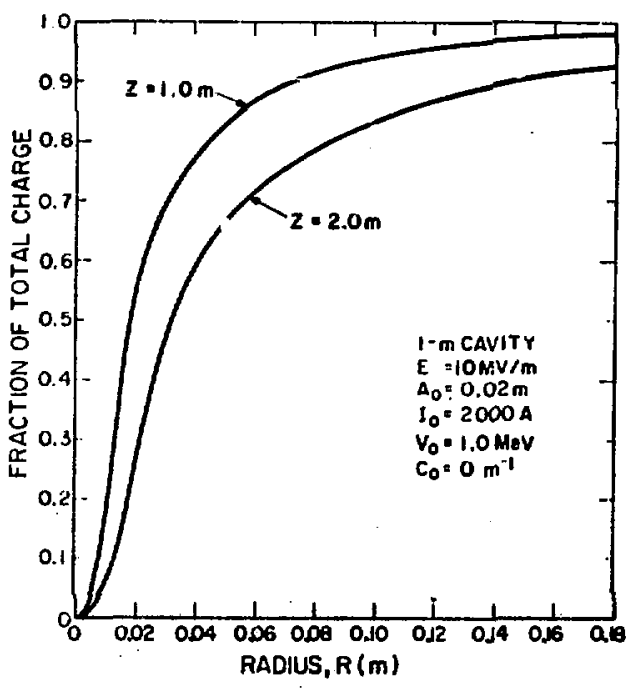

Fig. B-101.

Fraction of total charge hitting downstream wall within radius $R$.

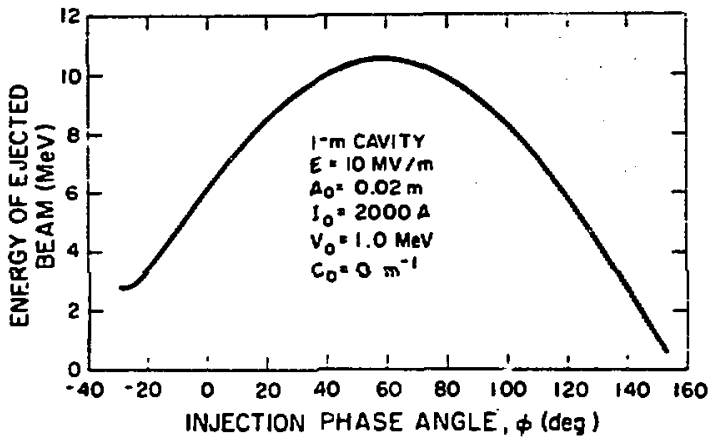

Fig. B-102.

Energy of ejected beam vs injection phase angle.

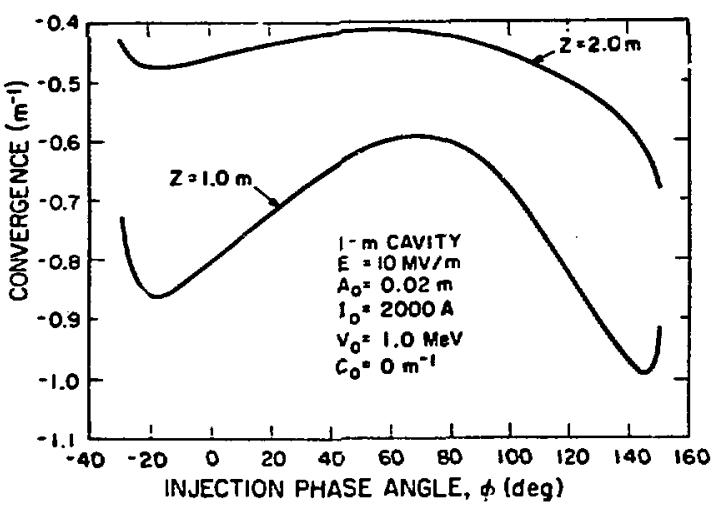

Fig. B-103.

Convergence of ejected beam vs injection phase angle.

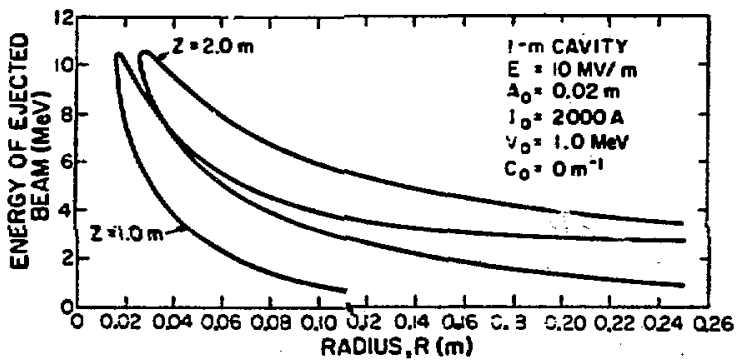

Fig. B-104.

Energy of ejected beam vs radius. 


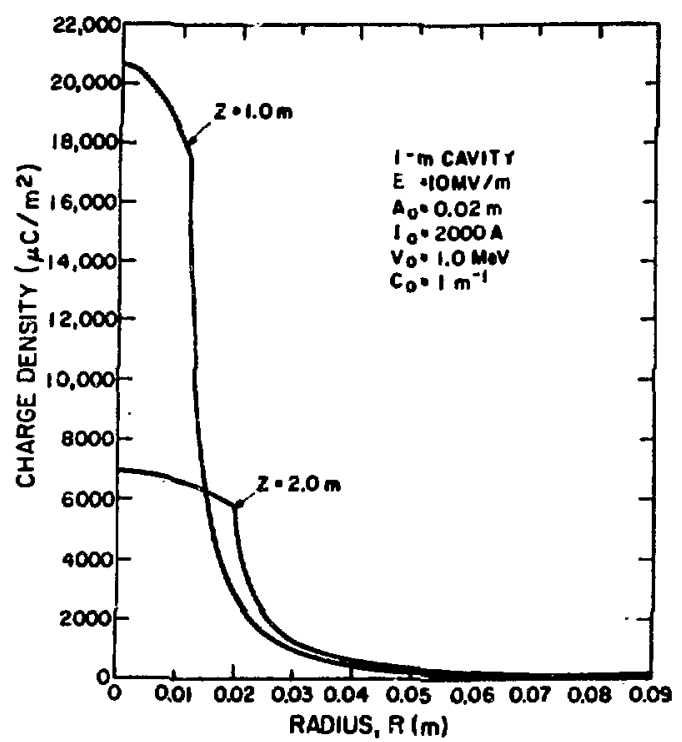

Fig. B-105.

Charge density vs radius.

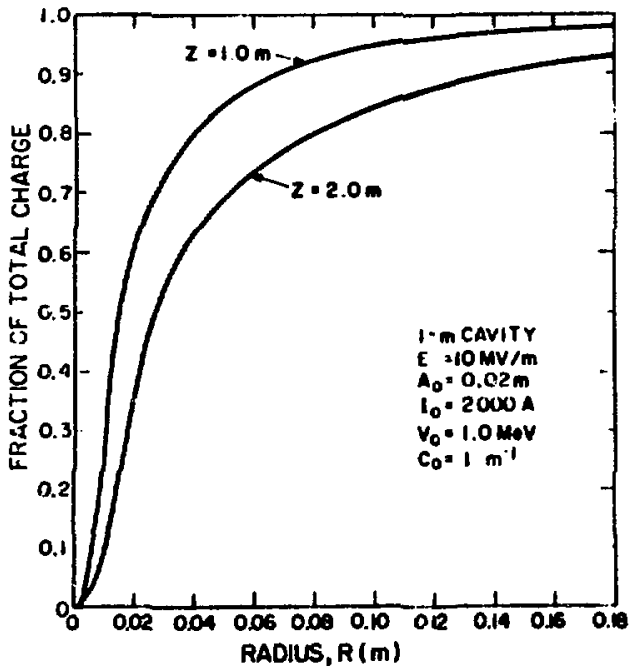

Fig. B-106.

Fraction of total charge hitting downstream wall within radius $R$.

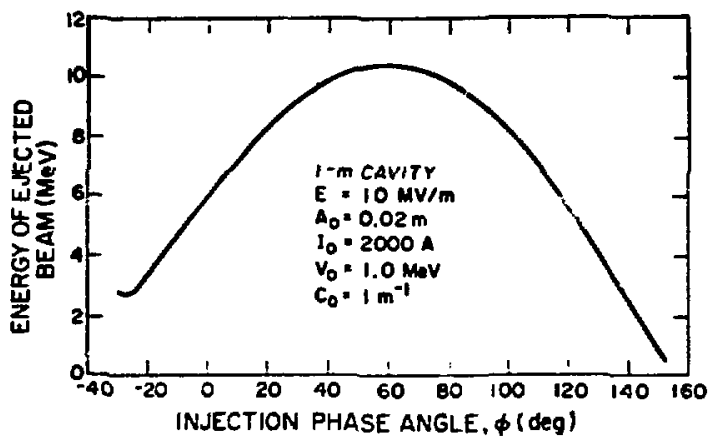

Fig. B-107.

Energy of ejected beam vs injection phase angle.

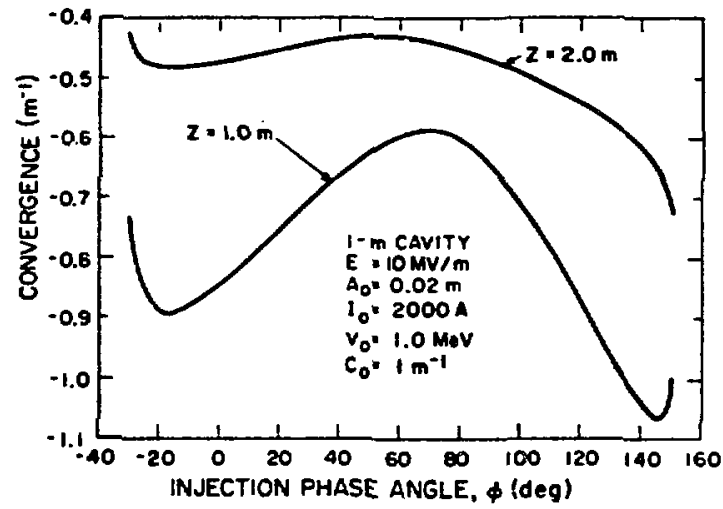

Fig. B-108.

Convergence of ejected beam vs injection phase angle.

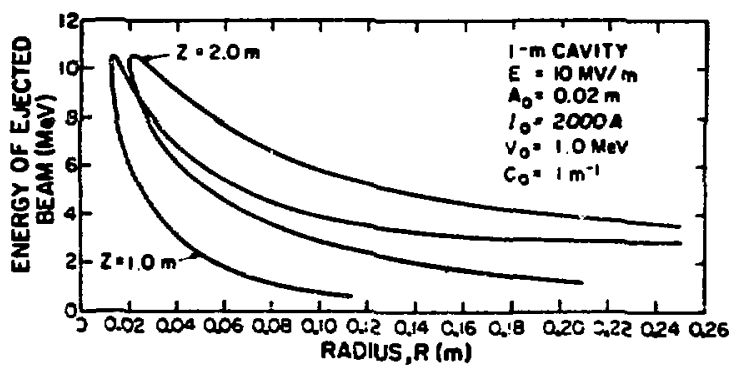

Fig. B-109.

Energy of ejected beam vs radius. 


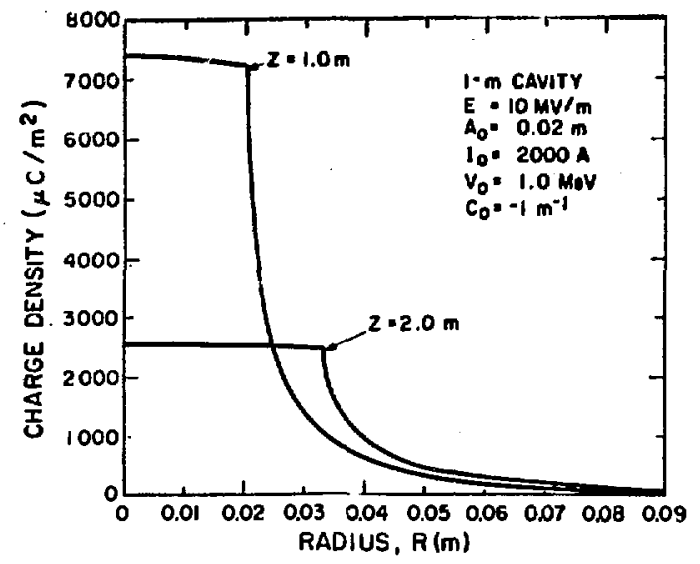

Fig. B-110.

Charge density vs radius.

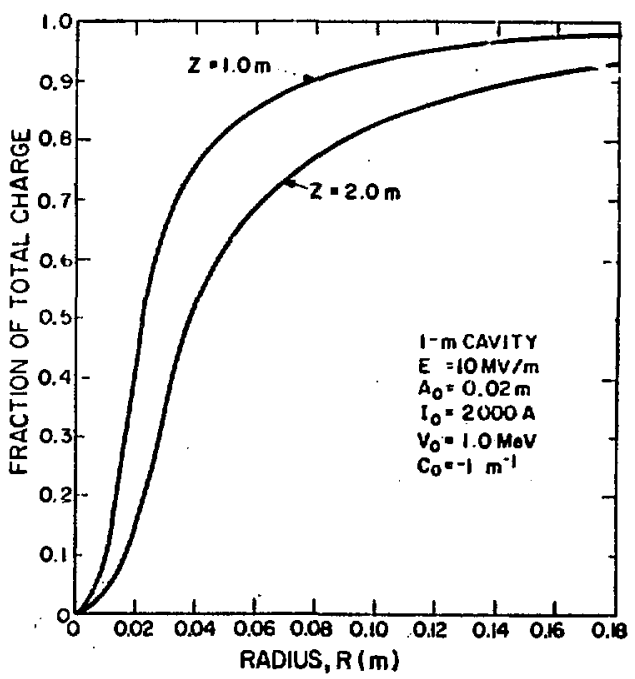

Fig. B-111.

Fraction of total charge hitting downstream wall within radius $R$.

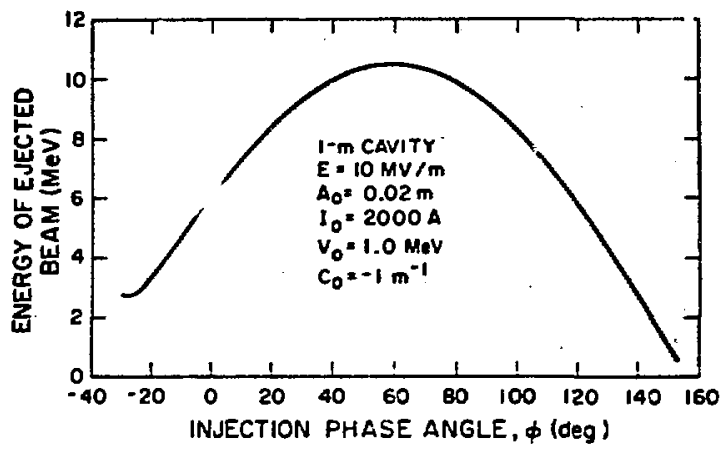

Fig. B-112.

Energy of ejested beam vs injection phase angle.

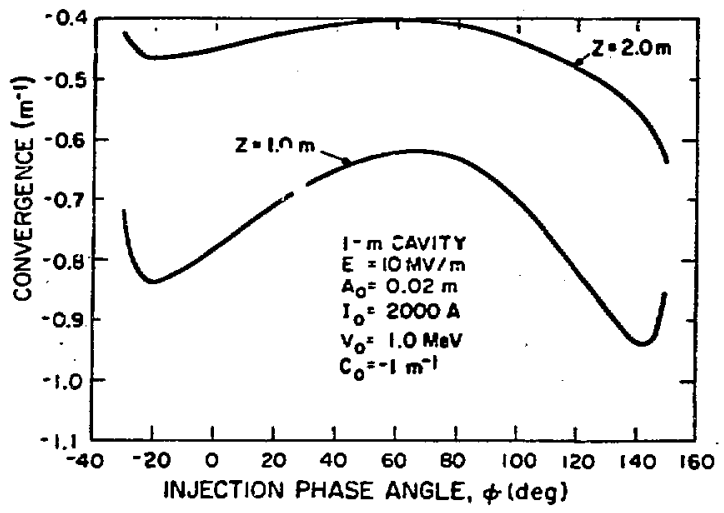

Fig. B-113.

Convergence of ejected beam vs injection phase angle.

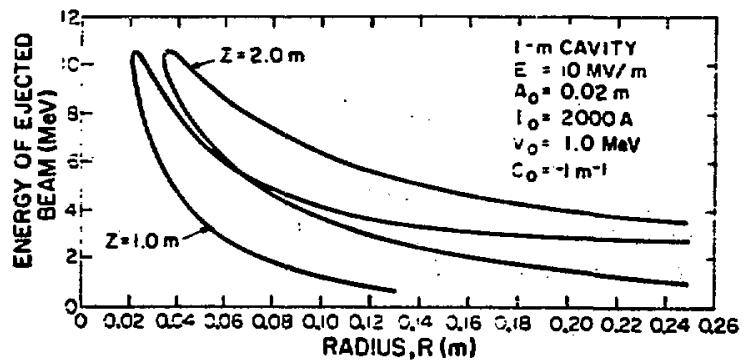

Fig. B-114.

Energy of ejected beam vs radius. 


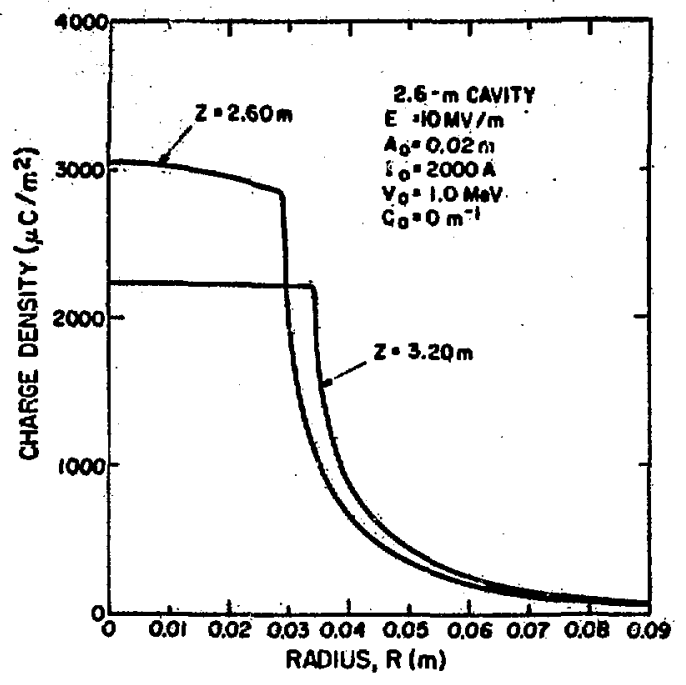

Fig. 8-115.

Charge density vs radius.

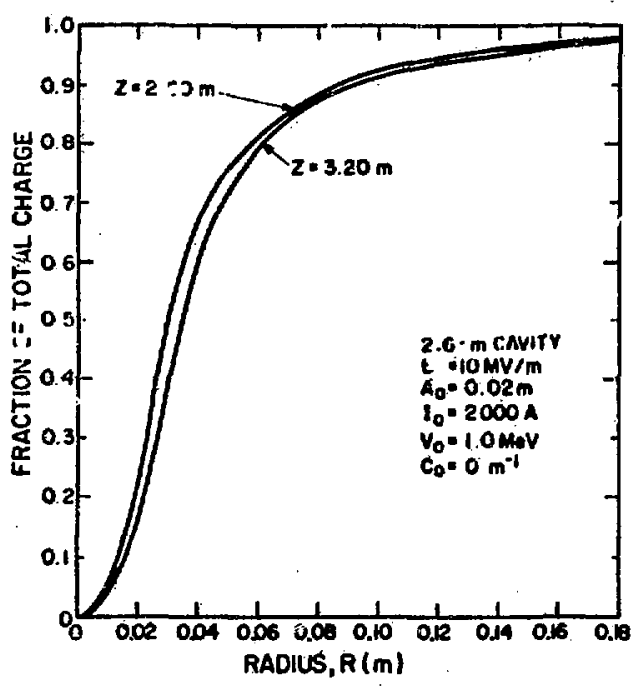

Fig. B-116.

Fraction of total charge hitting downstream wall within radius $R$.

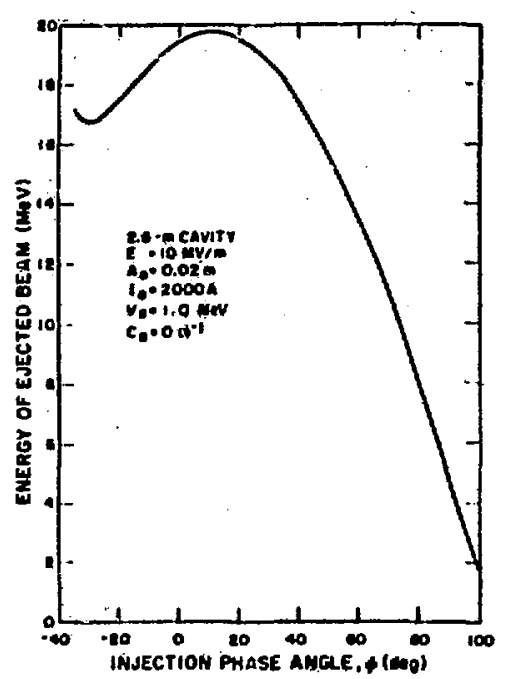

Fig. B-117.

Energy of ejecrad beam vs injection phase angle.

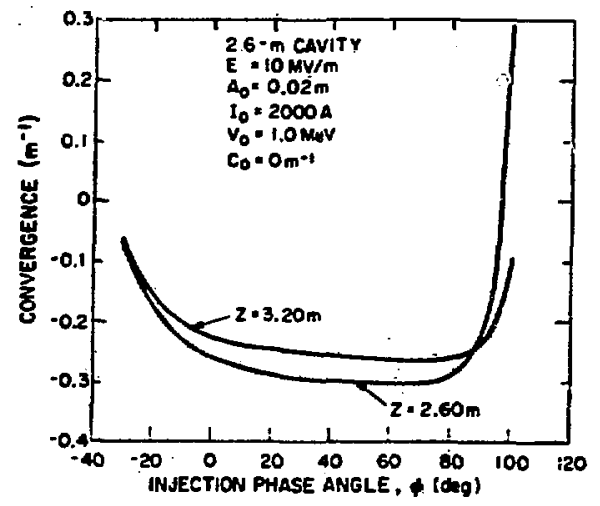

Fig. $B-118$.

Convergence of ejected beam vs injection phase angle.

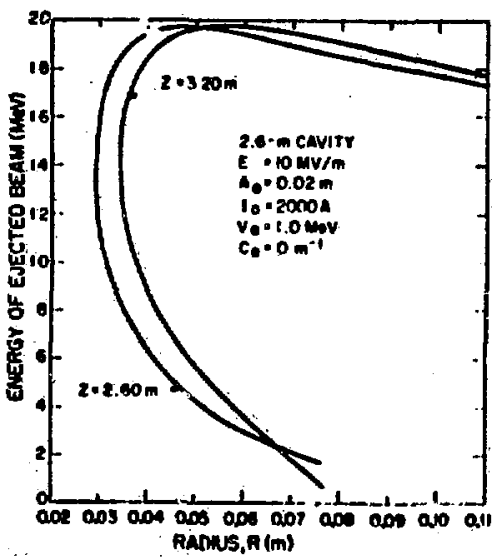

Rinus, $\mathrm{A}$ (m)
Fig. B-119:

Energy of ejected beam vs radlus. 


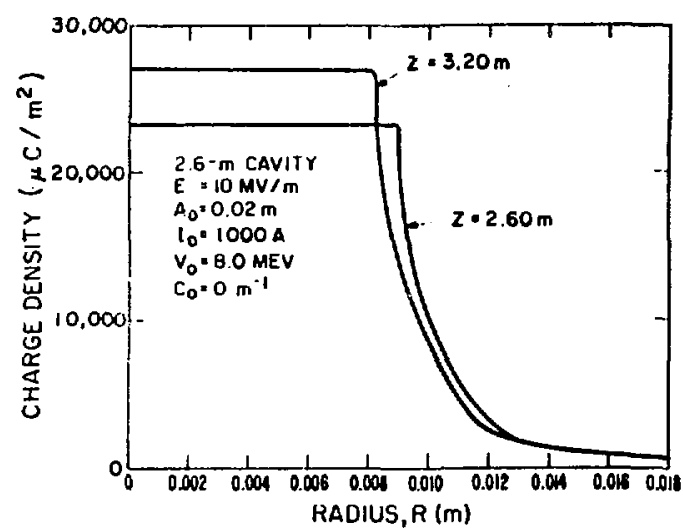

Fig. B-120.

Charge density vs radius.

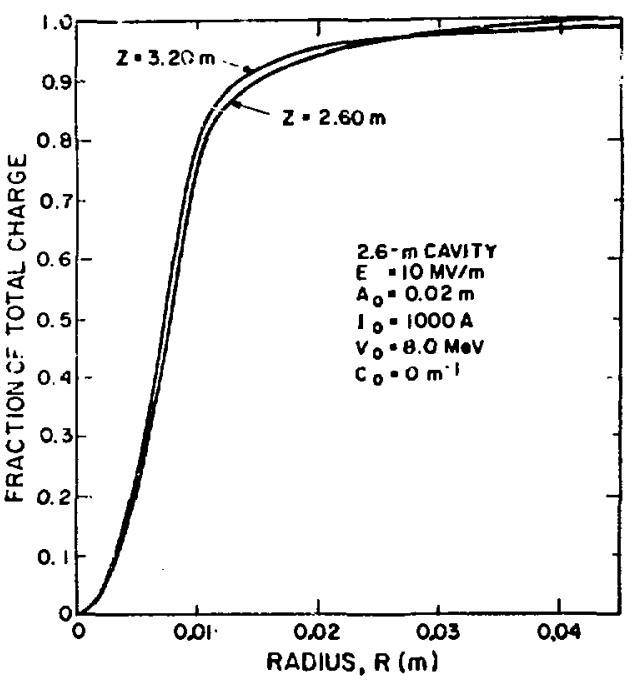

Fig. B-121.

Fraction of total charge hitting downstream wall within radius $R$.

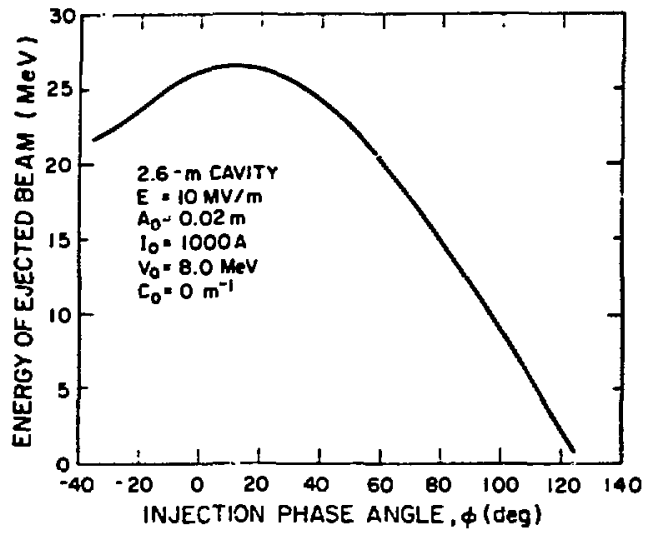

Fig. B-122.

Enorgy of ejected beam vs injection phase ancle

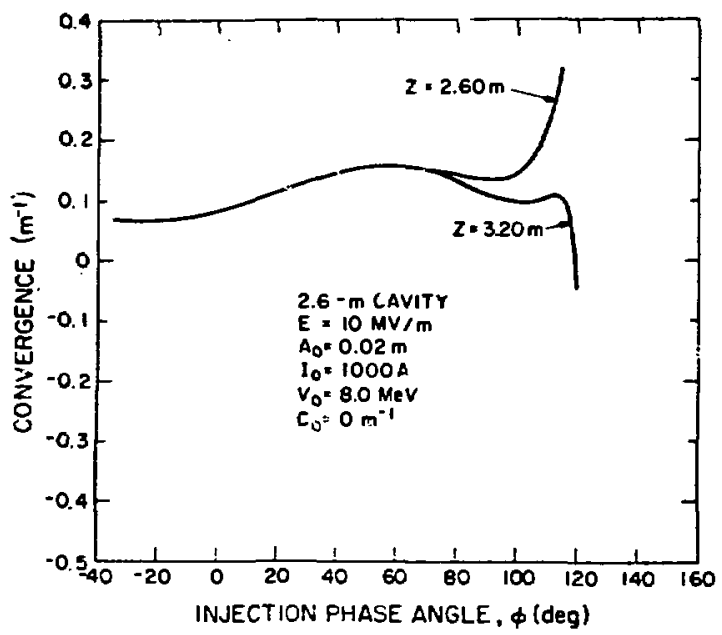

Fig. B-123.

Corvifrgence of ejected beam vs injection phase angle.

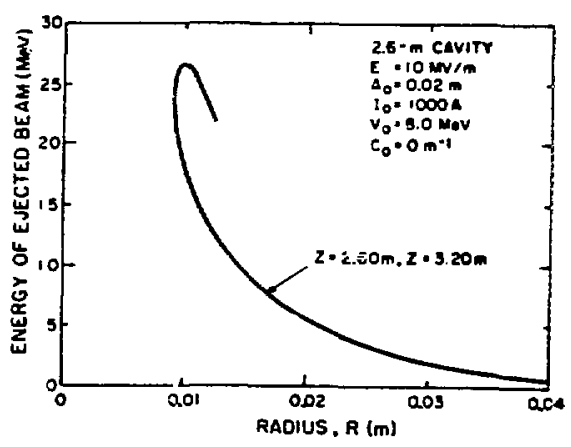

Fig. $B-12$ s.

Energy of ejected heam vs radius. 


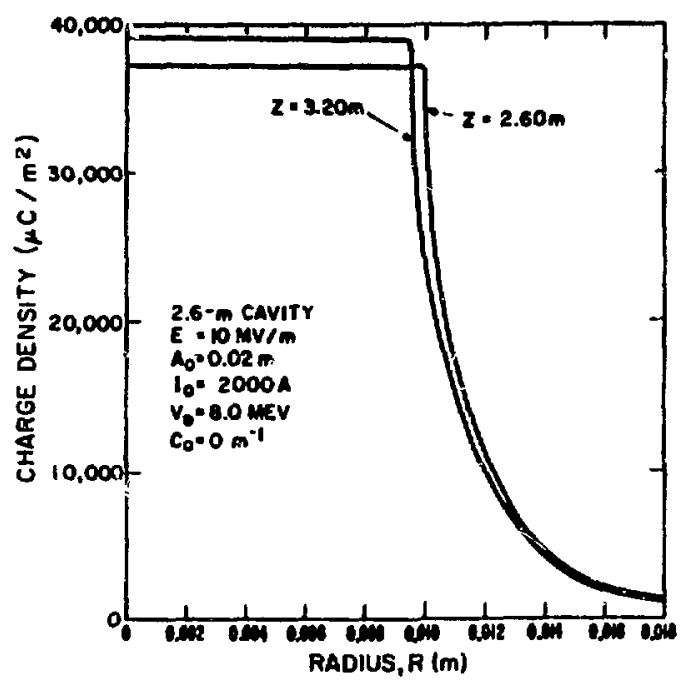

Fig. B-125.

Charge density vs radius.

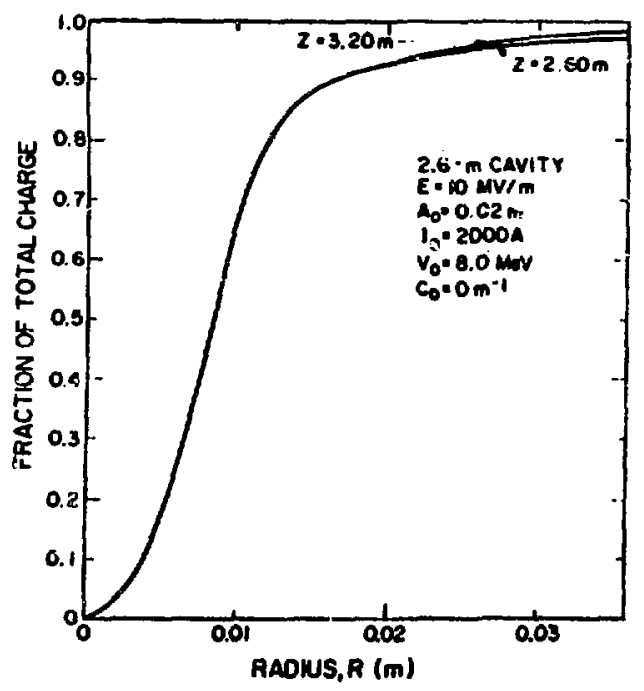

Fig. B-126.

Fraction of total charge hitting downstream wall within radius $R$.

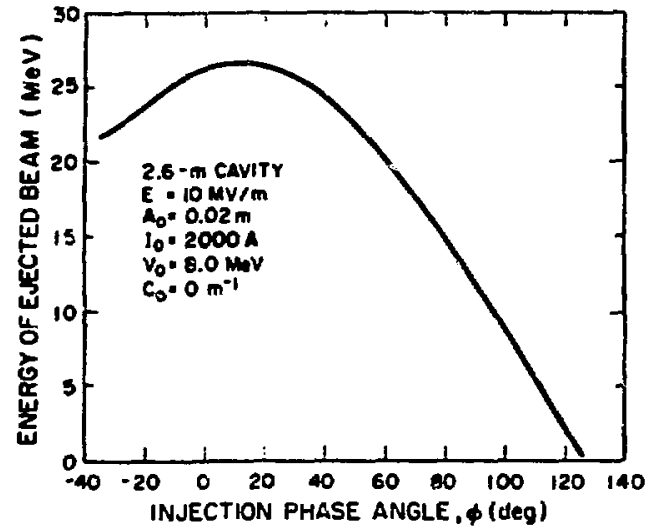

Fig. B-127.

Energy of ejacted beam vs injection phase angle.

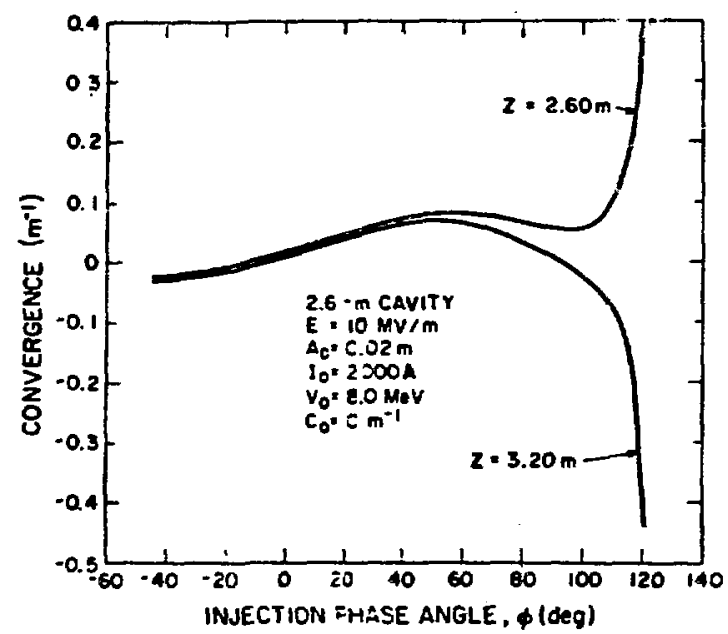

Fig. B-128.

Convergence of ejected beam vs injection phase angle.

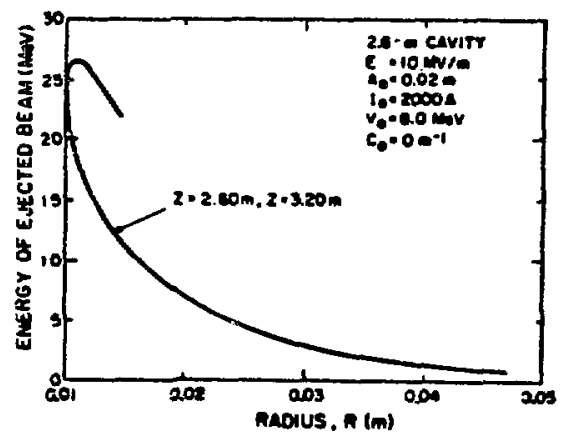

Fig. B-129.

Energy of ejected beam vs radius. 


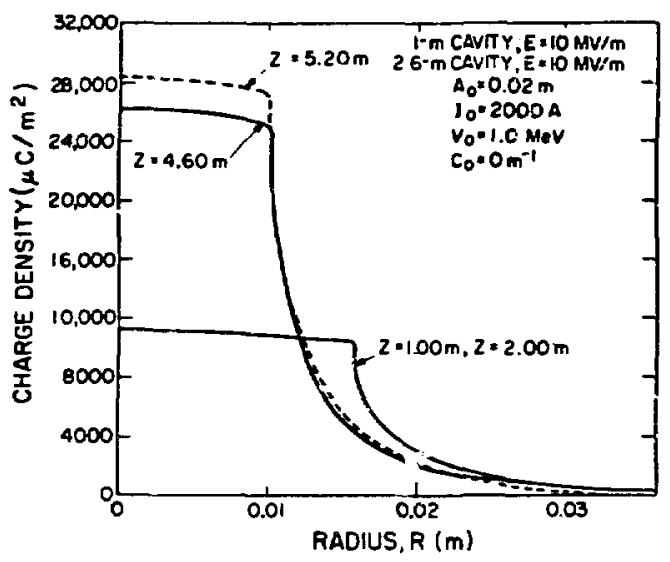

Fig. B-130.

Charge density vs radius.

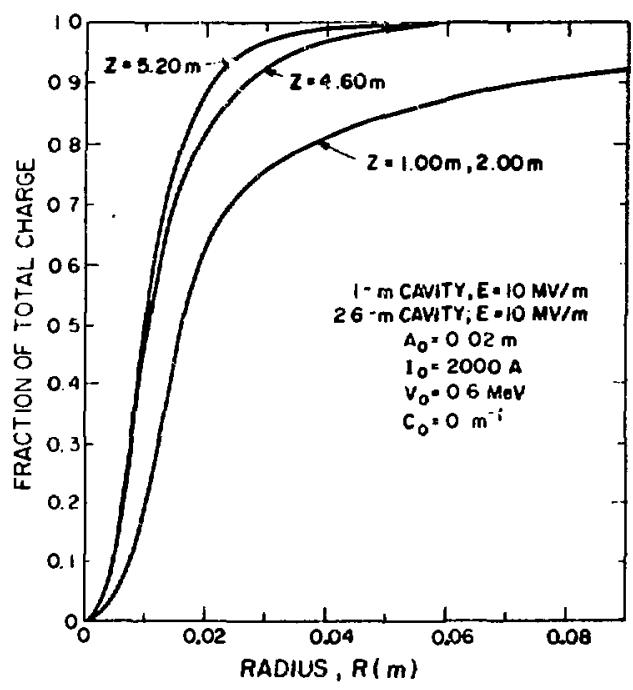

Fig. B-131.

Fraction of total charge hitting downstream wall within radius $A$.

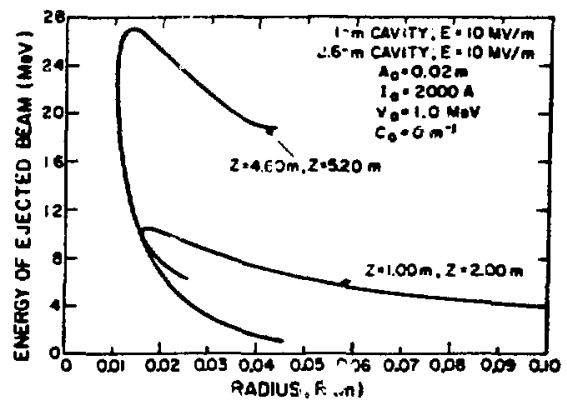

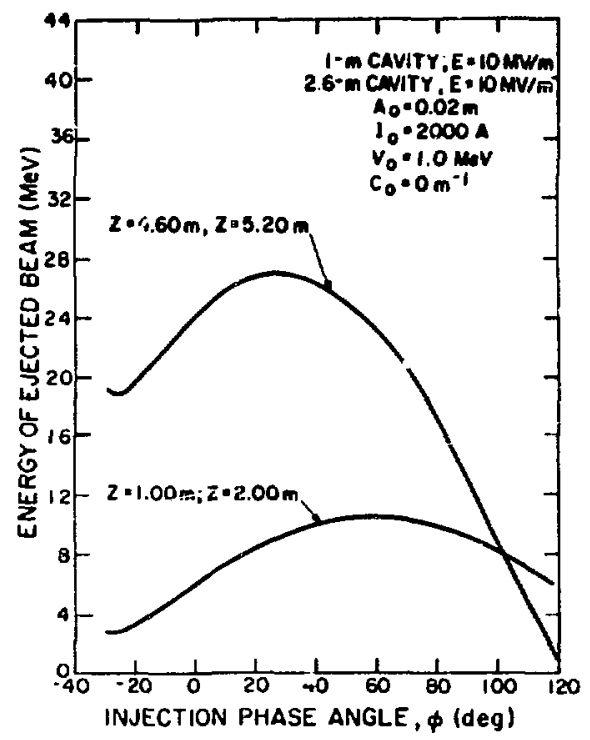

Fig. B-132.

Energy of ejected beam vs iniection phase angle.

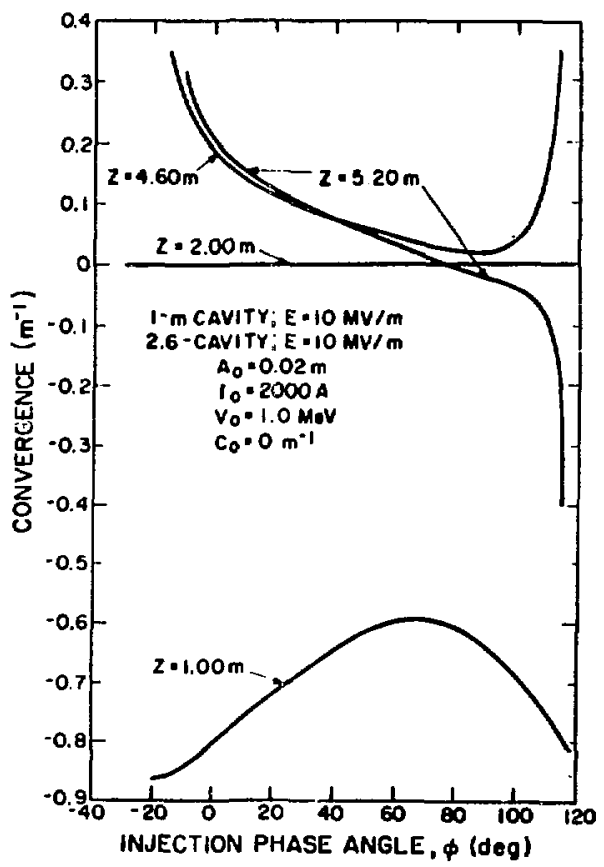

Fig. B-133.

Convergence of ejected beam vs injection phasa angle.

Fig. B-134.

Energy of ejected beam vs radius. 